\title{
Article \\ Exploration of Extended Higgs Sectors with Run-2 Proton-Proton Collision Data at the LHC
}

\author{
Arnaud Ferrari ${ }^{1, *(D)}$ and Nikolaos Rompotis ${ }^{2}$ (D) \\ 1 Department of Physics and Astronomy, Uppsala University, 75120 Uppsala, Sweden \\ 2 Department of Physics, University of Liverpool, Liverpool L69 3BX, UK; Nikolaos.Rompotis@liverpool.ac.uk \\ * Correspondence: arnaud.ferrari@physics.uu.se
}

check for updates

Citation: Ferrari, A.; Rompotis, N. Exploration of Extended Higgs Sectors with Run-2 Proton-Proton Collision Data at the LHC. Symmetry 2021, 13, 2144. https://doi.org/ $10.3390 /$ sym 13112144

Academic Editor: Gui Jun Ding

Received: 26 October 2021

Accepted: 5 November 2021

Published: 10 November 2021

Corrected: 28 July 2022

Publisher's Note: MDPI stays neutral with regard to jurisdictional claims in published maps and institutional affiliations.

Copyright: (C) 2021 by the authors. Licensee MDPI, Basel, Switzerland. This article is an open access article distributed under the terms and conditions of the Creative Commons Attribution (CC BY) license (https:// creativecommons.org/licenses/by/ $4.0 /)$.

\begin{abstract}
One doublet of complex scalar fields is the minimal content of the Higgs sector in order to achieve spontaneous electroweak symmetry breaking and, in turn, to generate the masses of fundamental particles in the Standard Model. However, several theories beyond the Standard Model predict a nonminimal Higgs sector and introduce additional singlets, doublets or even higher-order weak isospin representations, thereby yielding additional Higgs bosons. With its high proton-proton collision energy (13 TeV during Run-2), the Large Hadron Collider opens a new window towards the exploration of extended Higgs sectors. This review article summarises the current state-of-the-art experimental results recently obtained in searches for new neutral and charged Higgs bosons with a partial or full Run-2 dataset.
\end{abstract}

Keywords: physics beyond the Standard Model; Higgs bosons; LHC; ATLAS; CMS

\section{Introduction}

After the discovery of a Higgs boson with a mass of $125 \mathrm{GeV}$ [1-3] at the Large Hadron Collider (LHC) [4] in 2012, one of the main physics goals of the ATLAS [5] and CMS [6] experiments is to search for additional neutral and charged Higgs bosons. In the Standard Model (SM), electroweak symmetry breaking [7-12] occurs in a minimal way (i.e., only one complex scalar doublet is introduced). On the other hand, most theories beyond the SM enrich the Higgs sector with additional singlets, doublets and/or triplets of real or complex scalar fields, in turn predicting new physical spin-zero states, some neutral and some with an electric charge. Therefore, the observation of new neutral and charged Higgs bosons at the LHC would unequivocally prove the existence of new physics, beyond the SM.

This review article summarises the current state-of-the-art experimental results obtained by the ATLAS and CMS experiments in searches for new neutral and charged Higgs bosons based on a partial or full LHC Run-2 proton-proton collision dataset at a centre-ofmass energy $\sqrt{s}=13 \mathrm{TeV}$, recorded between 2015 and 2018. In Section 2, an overview of theoretical models with an extended Higgs sector is provided, together with their predictions in terms of additional spin-zero physical states. Then, Sections 3 and 4 summarise the searches for, respectively, neutral and charged Higgs bosons in the LHC Run-2 data. In Section 3, neutral Higgs boson searches are categorised according to final states arising from decays of these new spin-zero particles to either fermions or bosons. In Section 4, charged Higgs boson searches are categorised depending on the single- or double-electric charge that they carry. Finally, conclusions and outlooks are given in Section 5. In the following, generic symbols are used for all particles, and charge-conjugation is always implied.

\section{Extended Higgs Sectors}

The SM contains a Higgs sector that is composed of a weak isospin doublet of complex scalar fields. This is the simplest possible choice that is compatible with electroweak symmetry breaking and the structure of the weak nuclear and electromagnetic forces [13]. It is conceivable that the Higgs sector is more complicated. However, such a possibility 
is severely constrained by experimental measurements. In particular, the $\rho$ parameter of the electroweak theory, which at lowest order connects the masses of the $W$ and $Z$ bosons, respectively $m_{W}$ and $m_{Z}$, with the Weinberg angle, $\theta_{W}$ :

$$
\rho=\frac{m_{W}^{2}}{\cos ^{2} \theta_{W} m_{Z}^{2}},
$$

is measured to be very close to unity [14]. In general, a more complicated Higgs sector leads to significant deviations of $\rho$ from its measured value, thus rendering most options phenomenologically unviable or fine-tuned.

Some extensions of the Higgs sector survive the severe constraint from $\rho$. Most notably, extensions that add weak isospin singlets or doublets of fields leave the tree-level value of $\rho$ unaffected. The simplest of these extensions are the weak isospin singlet model [15-17] and the two-Higgs-doublet model $[18,19]$. Such theories introduce additional neutral and, in some cases, charged scalars, thereby leading to a much richer phenomenology than the SM. It is usually assumed that the masses of these additional scalars are at the same scale as the mass of the already discovered SM-like Higgs boson in order to avoid a new hierarchy problem within the Higgs sector. In turn, this feature makes a very strong case for searching for these additional Higgs bosons at the LHC.

\subsection{Singlet Extensions}

The simplest possible extension of the Higgs sector is obtained with the addition of a weak isospin singlet of scalar fields. If this singlet is real, there is just one additional Higgs boson with respect to the SM. This new Higgs boson, $H$, is typically considered to be heavier than the SM-like Higgs boson, $h$, which has a mass of about $125 \mathrm{GeV}$. The possibility of decay channels, such as $H \rightarrow W W / Z Z, H \rightarrow t t$, as well as $H \rightarrow h h$, is certainly very attractive in this model; however, there are tight constraints from electroweak precision measurements [20], $h$ coupling measurements [21,22] and $h h$ searches $[23,24]$. The case of the complex singlet extends the Higgs sector with two more scalar particles with respect to the SM content. The complex singlet model is possibly the simplest extension of the SM that can be tuned such that a dark matter candidate occurs $[25,26]$. In such a case, there is the option of Higgs bosons decaying to invisible particles such as $H / h \rightarrow A A$, where $A$ is one of the scalars that comes from the singlet. Finally, the singlet model is also relevant to electroweak baryogenesis scenarios [27]. It is the simplest model in which a strong, first-order electroweak phase transition evading the tight mass constraints that apply in the SM can occur. For such a phase transition to happen in the SM, the Higgs boson mass has to be less than about $80 \mathrm{GeV}$ [28], but this constraint is lifted if a nonminimal Higgs sector is considered. Currently, constraints from LHC measurements have disfavoured large parts of the available parameter space relevant for such scenarios; see, e.g., [29].

\subsection{The Two-Higgs-Doublet Model}

The two-Higgs-doublet model (2HDM) was originally introduced as a theory that realises spontaneous time-reversal symmetry violation [18]. Since then, it has enjoyed considerable attention because such a structure for the Higgs sector appears in supersymmetric models, such as the minimal supersymmetric extension of the SM (MSSM) [30], and it is necessary in several axion models [31]. Each of the two Higgs doublets of the 2HDM acquires a vacuum expectation value after electroweak symmetry breaking, and this leads to five physical Higgs bosons, two of which are charged and the remaining three are neutral. Under the very general assumptions of renormalisability and gauge symmetry conservation, the $2 \mathrm{HDM}$ comes with a vast parameter space comprising 14 free parameters [19]. For phenomenological studies, the model is simplified by assuming conservation 
of the CP symmetry and by ignoring quartic terms that are odd in one of the doublets. In this case, the 2HDM potential becomes:

$$
\begin{array}{r}
V=\sum_{i=1}^{2}\left(m_{i i}^{2}\left|\Phi_{i}\right|^{2}+\frac{\lambda_{i}}{2}\left|\Phi_{i}\right|^{4}\right)-m_{12}^{2}\left(\Phi_{1}^{\dagger} \Phi_{2}+\text { h.c. }\right)+\lambda_{3}\left|\Phi_{1}\right|^{2}\left|\Phi_{2}\right|^{2} \\
+\lambda_{4} \Phi_{1}^{\dagger} \Phi_{2} \Phi_{2}^{\dagger} \Phi_{1}+\frac{\lambda_{5}}{2}\left(\left(\Phi_{1}^{\dagger} \Phi_{2}\right)^{2}+\text { h.c. }\right)
\end{array}
$$

where the two complex Higgs doublets are denoted by $\Phi_{1}$ and $\Phi_{2}$, while $m_{11}, m_{22}, m_{12}, \lambda_{1}$, $\lambda_{2}, \lambda_{3}, \lambda_{4}$ and $\lambda_{5}$ are real-valued free parameters. By using the vacuum expectation value of the Higgs boson, which is known to be $v=246 \mathrm{GeV}$, the number of free parameters is reduced to seven. The assumption of $\mathrm{CP}$ conservation allows the neutral Higgs bosons to be CP eigenstates. In the following, the notations $h$ and $H$ are used for the CP-even Higgs bosons, assuming that $h$ is the lightest one, and $A$ for the CP-odd Higgs boson. The charged Higgs bosons are denoted by $H^{ \pm}$.

The interpretations of experimental results in the 2HDM parameter space do not usually employ the potential parameters of Equation (2). Instead, they consider the following equivalent free parameters: the masses of the Higgs bosons $m_{h}, m_{H}, m_{A}, m_{H^{ \pm}}$; the ratio of the vacuum expectation values of the two Higgs doublets, which is denoted as $\tan \beta$; and the mixing angle between the CP-even Higgs bosons $\alpha$, which is usually expressed as $\cos (\beta-\alpha)$ for reasons that will become clear later. The final free parameter, $m_{12}$, is fixed to some value-usually the value it takes in the MSSM-and it has little impact on the phenomenology of most search channels. For instance, Higgs boson decays to photons may be affected by $m_{12}$, which however plays little role in most searches for extended Higgs sectors. A general feature of the 2HDM that is also prominent after the previously discussed simplifications is the existence of flavour-changing neutral currents. This feature is severely constrained by experimental results; hence, one has either to fine-tune the model or include a mechanism to get rid of flavour-changing neutral currents. The common choice for the models in which experimental results tend to be interpreted is to use a 2HDM with built-in flavour conservation. This can be done by requesting that all fermions with the same chirality and the same weak isospin numbers couple to the same Higgs doublet [32,33]. By convention, the Higgs doublet $\Phi_{2}$ always couples to up-type quarks. This choice fixes the definition $\tan \beta=v_{2} / v_{1}$, where $v_{1}$ and $v_{2}$ are the vacuum expectation values of the $\Phi_{1}$ and $\Phi_{2}$ doublets, respectively. The couplings can then be arranged into four combinations, which are known as 2HDM type-I, type-II, lepton-specific and flipped, as shown in Table 1.

The 2HDM has weak and strong decoupling limits [34]. In the strong decoupling limit, the masses of $H, A$ and $H^{ \pm}$are much larger than $m_{h}$, and $\cos (\beta-\alpha)=0$. In this scenario, $h$ is indistinguishable from the SM Higgs boson. The weak decoupling limit, which is also known in the literature as the "alignment limit", is defined by $\cos (\beta-\alpha)=0$ only. In this limit, the lowest-order coupling expressions for $h$ and the SM Higgs boson are the same. However, higher-order corrections, as well as the existence of low mass Higgs bosons can reveal differences with respect to the SM.

A popular 2HDM scenario is that in which $h$ is taken to be the already discovered SM-like Higgs boson. This is not the only viable option; however, others are in general severely constrained; see, e.g., [35]. In this scenario, some typical 2HDM signatures for neutral Higgs bosons include $H \rightarrow h h, A \rightarrow Z h, A \rightarrow Z H$ and $H \rightarrow W W / Z Z$. It should be noted that, in the alignment limit, all the related couplings with the exception of $A Z H$ vanish; hence, they are disfavoured by precision measurements of the SM-like Higgs boson. The signature $A \rightarrow Z H$ is particularly interesting in the context of electroweak baryogenesis, since it allows for a strong, first-order electroweak phase transition [36]. In the type-II 2HDM, couplings to down-type fermions are enhanced at values of $\tan \beta>1$; hence, there is potential for $A / H \rightarrow \tau \tau$ and $A / H \rightarrow b b$ decays. The signature $A / H \rightarrow t t$ is also favoured in large parts of the parameter space. The production mechanism for the additional neutral Higgs bosons is similar to the SM Higgs boson, i.e., gluon-gluon 
fusion for all of them, and for $h$ and $H$, only vector boson fusion (VBF) and the associated production with a vector boson. In the type-II and flipped 2 HDM at $\tan \beta>1$, Higgs boson production in association with $b$-quarks is enhanced and becomes dominant.

Table 1. Coupling coefficients of the neutral bosons $h, H$ and $A$ to fermions (top) and vector bosons (bottom). By convention, the Yukawa couplings are the same for up-type quarks $(u)$ and may differ for down-type quarks $(d)$ and charged leptons $(\ell)$ depending on the 2HDM type for models with built-in flavour conservation. These coefficients $\xi$ are defined such that the Yukawa Lagrangian terms are $-\left(m_{f} / v\right) \bar{f} f \phi$ and $i\left(m_{f} / v\right) \bar{f} \gamma_{5} f A$ where $f=u, d, \ell$ and $\phi=h, H$. The coupling coefficients to vector bosons are defined such that the Feynman rule for the coupling $\phi V V$ is given by $2 \operatorname{im}_{V}^{2} \xi(\phi, V V) g_{\mu \nu} / v$, where $\phi=h, H, A$ and $V=W, Z$. The notations $s_{\beta-\alpha}, c_{\beta-\alpha}$ and $t_{\beta}$ refer to $\sin (\beta-\alpha), \cos (\beta-\alpha)$ and $\tan \beta$, respectively.

\begin{tabular}{|c|c|c|c|c|}
\hline Yukawa Couplings & Type-I & Type-II & Lepton-Specific & Flipped \\
\hline $\begin{array}{l}\xi(h, u) \\
\xi(h, d) \\
\xi(h, \ell)\end{array}$ & $\begin{array}{l}s_{\beta-\alpha}+c_{\beta-\alpha} / t_{\beta} \\
s_{\beta-\alpha}+c_{\beta-\alpha} / t_{\beta} \\
s_{\beta-\alpha}+c_{\beta-\alpha} / t_{\beta}\end{array}$ & $\begin{array}{l}s_{\beta-\alpha}+c_{\beta-\alpha} / t_{\beta} \\
s_{\beta-\alpha}-c_{\beta-\alpha} \cdot t_{\beta} \\
s_{\beta-\alpha}-c_{\beta-\alpha} \cdot t_{\beta}\end{array}$ & $\begin{array}{l}s_{\beta-\alpha}+c_{\beta-\alpha} / t_{\beta} \\
s_{\beta-\alpha}+c_{\beta-\alpha} / t_{\beta} \\
s_{\beta-\alpha}-c_{\beta-\alpha} \cdot t_{\beta}\end{array}$ & $\begin{array}{l}s_{\beta-\alpha}+c_{\beta-\alpha} / t_{\beta} \\
s_{\beta-\alpha}-c_{\beta-\alpha} \cdot t_{\beta} \\
s_{\beta-\alpha}+c_{\beta-\alpha} / t_{\beta}\end{array}$ \\
\hline $\begin{array}{l}\xi(H, u) \\
\xi(H, d) \\
\xi(H, \ell) \\
\end{array}$ & $\begin{array}{l}c_{\beta-\alpha}-s_{\beta-\alpha} / t_{\beta} \\
c_{\beta-\alpha}-s_{\beta-\alpha} / t_{\beta} \\
c_{\beta-\alpha}-s_{\beta-\alpha} / t_{\beta} \\
\end{array}$ & $\begin{array}{l}c_{\beta-\alpha}-s_{\beta-\alpha} / t_{\beta} \\
c_{\beta-\alpha}+s_{\beta-\alpha} \cdot t_{\beta} \\
c_{\beta-\alpha}+s_{\beta-\alpha} \cdot t_{\beta} \\
\end{array}$ & $\begin{array}{l}c_{\beta-\alpha}-s_{\beta-\alpha} / t_{\beta} \\
c_{\beta-\alpha}-s_{\beta-\alpha} / t_{\beta} \\
c_{\beta-\alpha}+s_{\beta-\alpha} \cdot t_{\beta}\end{array}$ & $\begin{array}{l}c_{\beta-\alpha}-s_{\beta-\alpha} / t_{\beta} \\
c_{\beta-\alpha}+s_{\beta-\alpha} \cdot t_{\beta} \\
c_{\beta-\alpha}-s_{\beta-\alpha} / t_{\beta}\end{array}$ \\
\hline $\begin{array}{l}\xi(A, u) \\
\xi(A, d) \\
\xi(A, \ell)\end{array}$ & $\begin{array}{c}1 / t_{\beta} \\
-1 / t_{\beta} \\
-1 / t_{\beta}\end{array}$ & $\begin{array}{c}1 / t_{\beta} \\
t_{\beta} \\
t_{\beta}\end{array}$ & $\begin{array}{c}1 / t_{\beta} \\
-1 / t_{\beta} \\
t_{\beta}\end{array}$ & $\begin{array}{c}1 / t_{\beta} \\
t_{\beta} \\
-1 / t_{\beta}\end{array}$ \\
\hline & $\begin{array}{r}\text { Coupli } \\
\text { (commo } \\
\xi(h, V V) \\
\xi(H, V V) \\
\xi(A, V V)\end{array}$ & $\begin{array}{l}\text { igs to Vector Bos } \\
\text { n to all 2HDM ty }\end{array}$ & $\begin{array}{l}\text { sons } \\
\text { pes) } \\
s_{\beta-\alpha} \\
c_{\beta-\alpha} \\
0\end{array}$ & \\
\hline
\end{tabular}

In the type-II and flipped 2HDM, charged Higgs bosons are produced in top-quark decays, $t \rightarrow H^{ \pm} b$, if they are light enough, or in association with $b$ - or $t$-quarks. Light charged Higgs bosons (i.e., with $m_{H^{ \pm}}$smaller than the top-quark mass) decay primarily to $\tau v$, whereas $H^{ \pm} \rightarrow t b$ dominates at higher masses. For low $m_{H^{ \pm}}$and low $\tan \beta, H^{ \pm} \rightarrow c s$ is also relevant. Light charged Higgs boson decays to quarks, $H^{ \pm} \rightarrow c s / c b$, are more relevant in the type-I and lepton-specific 2HDM. Another possible decay is $H^{ \pm} \rightarrow W h / W H$ : the relevant coupling is the same in all types of $2 \mathrm{HDM}$, but it may be more relevant in the type-I 2HDM because the other partial widths tend to be smaller.

It is possible to develop 2HDM scenarios in which the requirement of CP conservation is relaxed. The effect of CP violation depends on a single parameter, which is tightly constrained by the coupling measurements of the SM-like Higgs boson [37]. There is, nevertheless, available parameter space for such a model, and it can be distinguished from the CP-conserving 2HDM by targeting decay chains of heavy Higgs bosons into lighter ones. As an illustration, a decay $h_{3} \rightarrow h_{1} h_{2}$ followed by $h_{2} \rightarrow Z h_{1}$ implies that the physical state $h_{2}$ is CP-odd, in turn meaning that also $h_{3}$ is CP-odd, which leads to CP-violation in the context of $2 \mathrm{HDM}$.

The MSSM has a Higgs sector structure that is of type-II 2HDM [30]. In the MSSM, supersymmetry fixes the values of many of the free parameters of the 2HDM potential. In particular, only $m_{A}$ and $\tan \beta$ remain free, whereas the remaining parameters are fixed once the supersymmetry-related parameters are given. The largest effect on the Higgs bosons comes from third-generation squarks, as well as Higgsino and gaugino mass parameters. Fixing the supersymmetry-related parameters to various values yields a series of MSSM Higgs boson scenarios [38-40], which have evolved with time to keep up with the experimental constraints, as well as the mass and coupling measurements of the SM-like Higgs 
boson at the LHC. For example, in the $\mathrm{M}_{h}^{125}$ scenario [40], all supersymmetric particles are chosen to be heavy, so that the Higgs sector is only mildly affected by their presence, leading to a phenomenology that resembles the type-II 2HDM. In addition stop mixing is chosen such that the mass of the lightest $\mathrm{CP}$-even Higgs boson is maximised, resulting in large parts of the parameter space being compatible with a mass of $125 \mathrm{GeV}$. Another approach is to use a simplifying approach, as in the case of the "hMSSM scenario" [41-44]. This approach makes several assumptions, including that all supersymmetric particles are heavy and that the radiative corrections can be expressed in terms of the parameters that enter at tree level. As a result, the mass of the lightest CP-even Higgs boson can be fixed at $125 \mathrm{GeV}$. Despite the fact that several assumptions of this approximation are not valid for the whole parameter space, the simplicity of this approach has led the ATLAS and CMS collaborations to include it in their interpretations. In general, for most of these scenarios or approximations, the relevant signatures resemble those from the type-II 2HDM previously discussed for both neutral and charged Higgs bosons.

\subsection{Beyond the $2 H D M$}

Extensions of the $2 \mathrm{HDM}$ where a weak isospin singlet is added $(2 \mathrm{HDM}+\mathrm{S})[45,46]$ are popular, mainly because such a construction appears in the next-to-minimal supersymmetric SM (NMSSM) [47]. The main feature of $2 \mathrm{HDM}+\mathrm{S}$ that has been exploited by experiments is the prediction of light $(<100 \mathrm{GeV})$ pseudoscalar particles, $a$, that can decay into a plethora of kinematically allowed final states: $a \rightarrow e e, \mu \mu, \tau \tau, \gamma \gamma, q q, g g$. These particles can be produced directly, via, e.g., gluon-gluon fusion, or through Higgs boson decays, $h \rightarrow a a$ or $h \rightarrow Z a$. In addition, light pseudoscalar production via charged Higgs boson decays, $H^{ \pm} \rightarrow W a$, is also possible [48-51].

All the extensions discussed so far include weak isospin singlets and doublets that automatically satisfy the requirement that the $\rho$ parameter be one at the tree level. It is possible, however, to use different weak isospin multiplets and fine-tune the model such that $\rho$ is still close to one. One such option is the Georgi-Machacek model [52,53], in which two weak isospin triplets, one real and the other complex, are added on top of the SM scalar doublet. These triplets have the same vacuum expectation value, and electroweak symmetry breaking leads to 10 Higgs bosons. The complexity of the Higgs sector allows for signatures such as $H^{ \pm} \rightarrow W Z$, which are not allowed in the 2HDM, and for $H \rightarrow W W / Z Z$, which may be allowed in $2 \mathrm{HDM}$ and singlet scenarios, but are severely constrained by SM-like Higgs boson coupling measurements. In addition, the Georgi-Machacek model predicts a doubly charged Higgs boson that, depending on the parameter choice, may decay to $H^{ \pm \pm} \rightarrow W^{ \pm} W^{ \pm}, \ell^{ \pm} \ell^{ \pm}$and $H^{ \pm} W^{ \pm}$. The Higgs bosons in this model are organised in a custodial symmetry fiveplet, one triplet and two singlets, each of them having physical states with the same mass. At the LHC experiments, a popular benchmark to interpret the results related to this model is to consider the plane of the fiveplet mass, $m_{H 5}$, and $s_{H}=\sin \theta_{H}$, which corresponds to an angle that relates the triplet and the SM vacuum expectation values [35]. It is usually assumed that the triplet mass is larger than the fiveplet mass, so $H_{5} \rightarrow \mathrm{ZZ} / \mathrm{WW}, H_{5}^{ \pm} \rightarrow W Z$ and $H_{5}^{ \pm \pm} \rightarrow W^{ \pm} W^{ \pm}$are the only allowed signatures.

\subsection{Theoretical Calculations Used in the Interpretation of Experimental Results}

Experimental results require theoretical calculations of the extended Higgs sector in order to be interpreted. Since 2010, the LHC Higgs Cross-Section Working Group-now known as the LHC Higgs Working Group-has undertaken the effort of providing theoretical predictions for SM and beyond-SM Higgs boson signatures. The results have been documented in a series of CERN Yellow Reports published in 2011 [54], 2012 [55], 2013 [56] and 2017 [35]. The ATLAS and CMS Collaborations generally follow the recommendations that are outlined in these reports.

For branching fractions, in general, the recommendation is to use HDECAY [57] or 2HDMC [58] for the 2HDM, HDECAY for the electroweak singlet model, ScannerS [20] for the $2 \mathrm{HDM}+\mathrm{S}$ scenario and GMCalc [59] for the Georgi-Machacek model. For the MSSM, a 
combination of different programs is used to obtain the best available predictions for each partial width, as explained in detail in [54]. For some specific MSSM benchmarks, HDECAY alone may be used [35].

For production cross-sections, in general, the recommendation is to use the best available prediction in terms of radiative corrections and to apply a modification on the beyond-SM couplings of the Higgs bosons, if needed. For VBF and associated productions of neutral Higgs bosons with a vector boson or heavy quarks, the SM Higgs boson calculations are used and subsequently scaled according to the couplings. For gluon-gluon fusion, a dedicated program, SusHi $[60,61]$, provides the cross-sections both for the 2HDM and the MSSM. The same program also provides calculations for the $b$-associated production in the five-flavour scheme, in which $b$-quarks appear in the initial state and are explicitly accounted for in the parton distribution functions. The recommendation, however, is to combine this result with the four-flavour scheme calculations $[62,63]$, where $b$-quarks are not included in the parton distribution functions and only appear in the final state (via, e.g., gluon splitting), following the prescription of [64] in the case of the 2HDM, or to use a more advanced calculation [65-68] in the case of the MSSM. For charged Higgs bosons, the only production mechanism that needs a dedicated calculation is the production in association with $t b$, as performed in [69-80]. There has also been recent progress in the calculation of $H^{ \pm}$production in the mass range around the top-quark mass, where the top-quark pair production and a subsequent top-quark decay to a $b$-quark and a charged Higgs boson interfere with the $H^{ \pm}$production in association with $t b$ [79].

\section{Searches for Neutral Higgs Bosons}

There is a large variety of searches for neutral Higgs bosons at the LHC. The flagship of such data analyses has been historically the search for a heavy $H / A \rightarrow \tau \tau$, due to the importance of this process in the MSSM. Similar channels, but with less sensitivity to the MSSM, include $H / A \rightarrow b b$ and $H / A \rightarrow \mu \mu$. Other searches for heavy neutral Higgs bosons at the LHC include those targeting decays to pairs of photons or weak bosons, $V V(V=W, Z)$. More focused on the 2HDM aspects of the phenomenology, though still relevant for the MSSM, is the search for $A \rightarrow Z h$, where $h$ denotes the SM-like Higgs boson with a mass of $125 \mathrm{GeV}$. In the interpretation of most of these search channels, it is usually assumed that both heavy neutral Higgs bosons have the same mass. This requirement is motivated by the MSSM, but also by the effect of electroweak precision measurements on the 2HDM, which implies that two of the three heavy Higgs bosons have to be nearly degenerate. However, the decay $A \rightarrow Z H$ evades these constraints and has been searched for in the $Z \rightarrow \ell \ell, H \rightarrow b b / W W / \tau \tau$ channels. Although a heavy resonance decaying to a pair of SM-like Higgs bosons is also a popular probe for additional neutral Higgs bosons, such signatures are not presented here, because they are covered in another dedicated review article in the same journal [81]. Finally, another way of probing extended Higgs sectors at the LHC is to search for new, light pseudoscalars, $a$, in exotic $125 \mathrm{GeV} \mathrm{Higgs}$ boson decays (e.g., $h \rightarrow a a$ ), where $a$ may decay to $\mu \mu / b b / \tau \tau / \gamma \gamma / g g$.

In the following, only some indicative examples of these searches will be reviewed. Heavy neutral Higgs bosons decaying to a fermion pair are discussed in Section 3.1, with emphasis on the $A / H \rightarrow \tau \tau$ channel. Searches for heavy Higgs bosons decaying to a pair of electroweak gauge bosons, $\gamma \gamma$ or $V V$, are discussed in Section 3.2, while searches featuring the decay of a pseudoscalar $A$ boson to a $Z$ boson and another, $C P$-even, Higgs boson are reviewed in Section 3.3. Finally, searches for light Higgs bosons, where "light" implies a mass below $125 \mathrm{GeV}$, are briefly mentioned in Section 3.4 .

\subsection{Searches for Heavy Neutral Higgs Bosons Decaying to a Fermion Pair}

Although other fermionic decays of heavy Higgs bosons will be briefly discussed in the following, this section mostly focuses on the search for $A / H \rightarrow \tau \tau$. In this data analysis, events are categorised according to the decay modes of the $\tau$-leptons. The latest ATLAS search [82] uses the full LHC Run-2 dataset of proton-proton collisions $\left(139 \mathrm{fb}^{-1}\right)$ 
to look for final states with at least one $\tau$-lepton decaying to hadrons. On the other hand, the latest CMS publication [83] uses only part of the proton-proton collision Run-2 dataset $\left(36 \mathrm{fb}^{-1}\right)$ and includes, in addition to the final states investigated by ATLAS, the decay chain $\tau \tau \rightarrow e \mu+$ neutrinos. The fully leptonic final state makes it easier to trigger the events, but it is penalised by low signal yields because the branching fraction of the $\tau \tau \rightarrow e \mu+$ neutrinos decay chain is only about $6 \%$. Another challenge is the presence of several neutrinos in the final state, which leads to a significant deterioration of the mass resolution.

The analysis strategy employed in both ATLAS and CMS searches is very similar. The $A / H \rightarrow \tau \tau$ search is optimised for the MSSM, which means that the intrinsic width of the neutral Higgs bosons is much smaller than the experimental resolution and the main production mechanisms are gluon-gluon fusion and in association with $b$-quarks. This motivates the separation of the analysis into "no $b$-tag" and " $b$-tag" categories, depending on the presence or not of at least an additional $b$-tagged jet.

The main backgrounds for this search are from $Z / \gamma^{*} \rightarrow \tau \tau$ and from processes with a quark- or gluon-initiated jet faking the hadronic decay of a $\tau$-lepton. The background estimation depends critically on data-driven techniques. Both experiments use a "fake factor" method for this purpose. In this method, a control region is defined, in which the probability of a quark- or gluon-initiated jet to be reconstructed and misidentified as a hadronic $\tau$-lepton decay is measured in the data as a function of several kinematic variables. Then, this probability is applied in another control region, defined such that the procedure provides an estimate of the background in the signal region. CMS also uses a so-called embedding technique in order to determine the $Z \rightarrow \tau \tau$ background by selecting a sample of $Z \rightarrow \mu \mu$ events in the data and subsequently replacing one or both muons by simulated $\tau$-lepton decays $[84,85]$.

The final results are obtained by fitting a version of the reconstructed $\tau \tau$ mass, $m_{T}^{\text {tot }}$, that takes into account the missing transverse momentum, defined as follows:

$$
m_{T}^{\text {tot }}=\sqrt{\left(p_{T 1}+p_{T 2}+p_{T}^{m i s s}\right)^{2}-\left(\vec{p}_{T 1}+\vec{p}_{T 2}+\vec{p}_{T}^{m i s s}\right)^{2}},
$$

where $p_{T 1}$ and $p_{T 2}$ are the magnitudes of the transverse momenta of the two reconstructed $\tau$-leptons and $p_{T}^{\text {miss }}$ is the magnitude of the missing transverse momentum. The same symbols with arrows denote the corresponding two-component vectors in the plane transverse to the LHC beam. Figure 1a shows an example of this distribution. More advanced mass reconstruction techniques have been used in some previous publications; see, for instance, [86]. However, these techniques are significantly slower to run and have some advantages mainly in the semileptonic or fully leptonic channels at the lower $\mathrm{A} / \mathrm{H}$ masses.

In the absence of any excess of data with respect to the SM predictions, search results are presented as the $95 \%$ confidence-level (CL) upper limits on the production cross-section of a narrow scalar particle as a function of its mass; see, e.g., Figure 1b. Interpretations have also been performed in various MSSM scenarios, as, e.g., in Figure 1c. In addition, in order to facilitate the use of the experimental results in phenomenological studies and with tools such as HiggsBounds [87], the two-dimensional likelihoods of the experimental fits are provided; see, e.g., Figure 1d. 


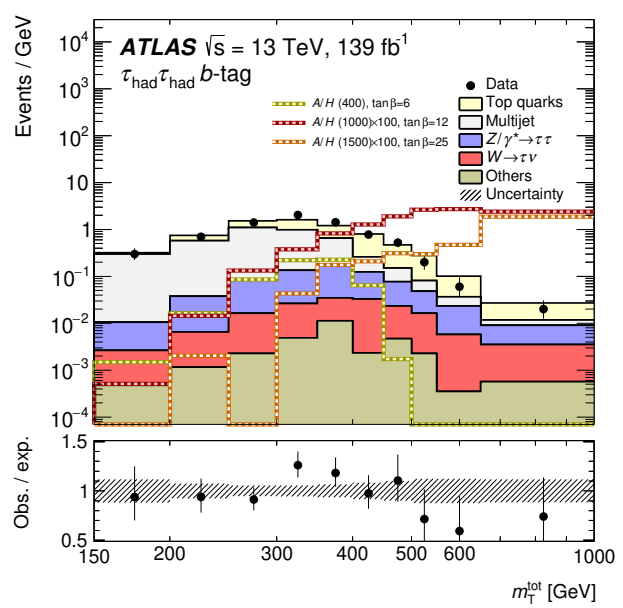

(a)

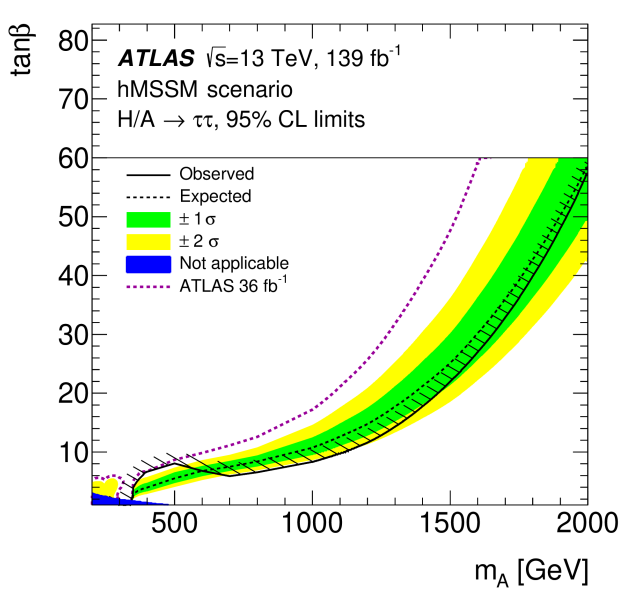

(c)

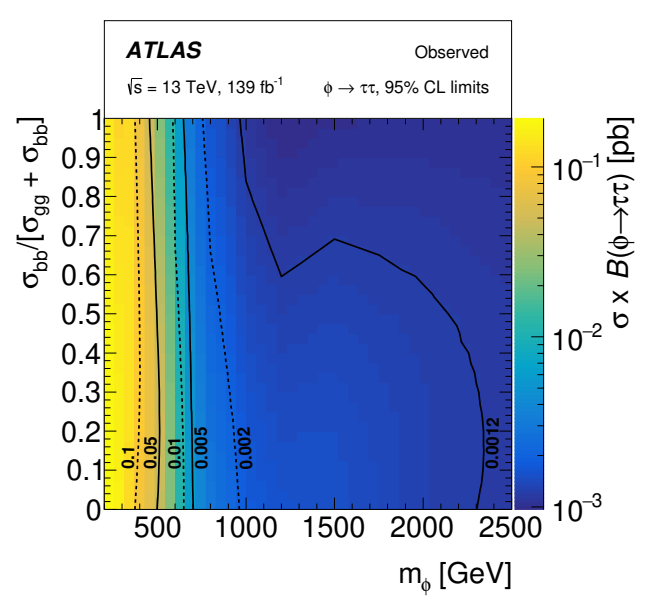

(b)
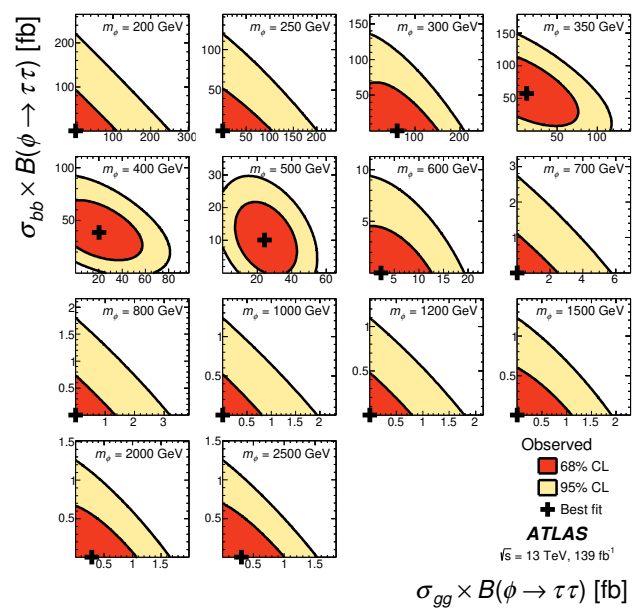

(d)

Figure 1. Results from the most recent ATLAS search for heavy neutral Higgs bosons decaying to $\tau \tau$ final states [88] showing: (a) the final discriminating variable, $m_{T}^{\text {tot }}$, for the " $b$-tag" event category in the final state where both $\tau$-leptons decay hadronically; (b) 95\% CL limits for the production cross-section times the branching fraction of a scalar boson $\phi \rightarrow \tau \tau$ as a function of its mass and the fraction of $b$-associated production; (c) the interpretation of the search in the $\left(m_{A}, \tan \beta\right)$ plane of the hMSSM; (d) scans of the likelihood function for a single narrow resonance produced via gluon-gluon fusion or in association with $b$-quarks. Copyright 2021 CERN for the benefit of the ATLAS Collaboration. CC-BY-4.0 license.

In addition to the searches in the $\tau \tau$ final states, other experimental signatures of heavy neutral Higgs bosons include the $b b$ and $\mu \mu$ decay channels. Related data analyses have been pursued by both ATLAS and CMS [89-92].

The $H / A \rightarrow b b$ search tends to be very difficult, with the main limitations arising from the trigger and the overwhelming multijet background processes. This decay mode is usually searched for in the $b$-associated production channel, where at least three jets are expected to contain a B-hadron ( $b$-jets). For example, in [89], ATLAS used both single and double $b$-jet triggers to select events. Along with the background considerations, the lowest mass that this search can have sensitivity to is ultimately $450 \mathrm{GeV}$, as illustrated by Figure 2 . The search tends to have lower sensitivity than the $\tau \tau$ channel in the MSSM. However, in the general 2HDM parameter space, there are regions where it may be competitive.

The $\mu \mu$ final state, in contrast to $b b$, is much easier to trigger on. Similar to the $A / H \rightarrow \tau \tau$ search, events are split into "no $b$-tag" and " $b$-tag" categories in order to target 
both gluon-gluon fusion and $b$-associated production. The backgrounds are smooth, and in general, parametric functional forms are used to fit the data. The excellent mass resolution of the dimuon system implies that this channel is more sensitive to effects from the natural width of the resonance, even in the case of the MSSM where the additional Higgs bosons tend to be narrow. Ultimately, this search channel suffers from the usually small expected branching fraction, and its sensitivity is in general lower than $A / H \rightarrow \tau \tau$ for most models. As an example, the most recent CMS search result is shown in Figure 3.

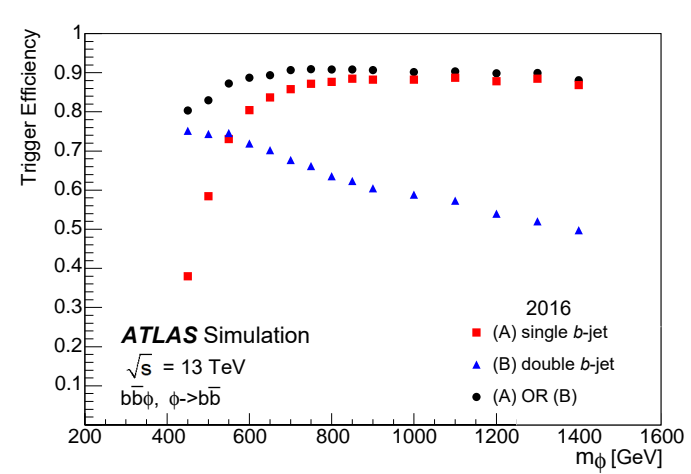

(a)

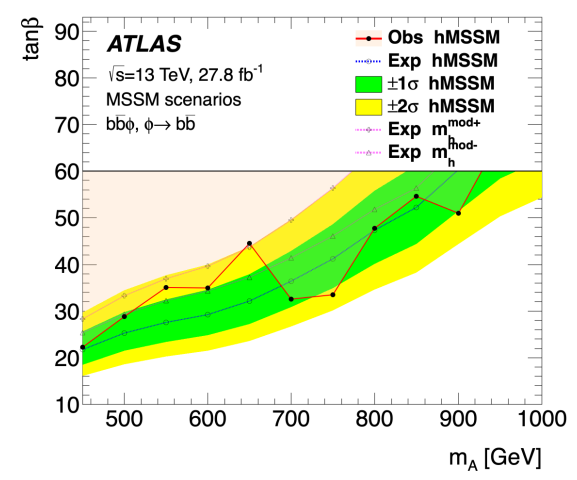

(b)

Figure 2. Results from an ATLAS search for $b$-associated production of a heavy neutral Higgs boson decaying to $b b$ [91] showing: (a) the efficiency of the single- and double- $b$-jet triggers as a function of the neutral Higgs boson mass for part of the dataset used in the analysis; (b) an interpretation of the search in the $\left(m_{A}, \tan \beta\right)$ plane of the hMSSM. Copyright 2021 CERN for the benefit of the ATLAS Collaboration. CC-BY-4.0 license.

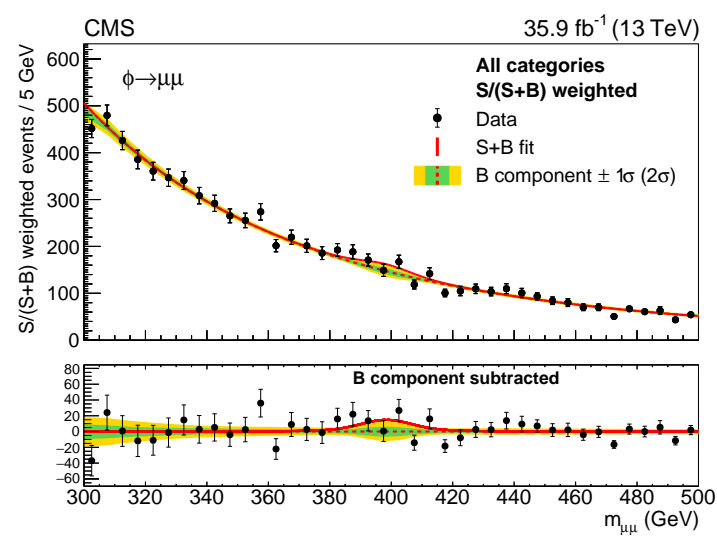

(a)

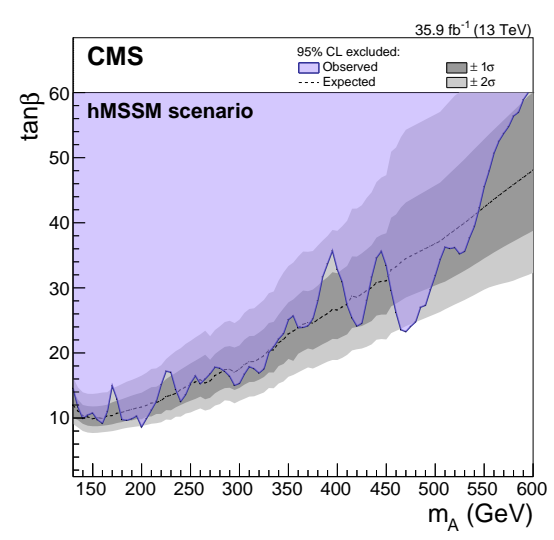

(b)

Figure 3. Results from a CMS search for a heavy neutral Higgs boson decaying to $\mu \mu$ [92] showing: (a) the example of a fit to the data with a signal+background hypothesis, for a narrow signal with a mass of $400 \mathrm{GeV}$ produced in association with $b$-quarks and decaying to two muons; (b) an interpretation of the search in the $\left(m_{A}, \tan \beta\right)$ plane of the hMSSM. Copyright 2021 CERN for the benefit of the CMS Collaboration. CC-BY-4.0 license.

Finally, it is possible to look for the direct production of a heavy Higgs boson decaying to a $t t$ pair. The most important production mechanism is gluon-gluon fusion. However, the $A / H \rightarrow t t$ signal process interferes with $\mathrm{SM} t t$ production, subsequently leading to a signal shape that does not resemble a usual resonance, as illustrated in Figure 4a. In turn, this reduces the sensitivity of the search. Both ATLAS and CMS have published searches for gluon-gluon produced $A / H \rightarrow t t$ in [93,94]; however, only CMS has performed this search with Run-2 data. Interpretations are provided in the context of the type-II 2HDM 
(ATLAS) and the hMSSM (CMS). In general, these searches are sensitive to low $\tan \beta$ values, up to about one, as shown in Figure 4 b.

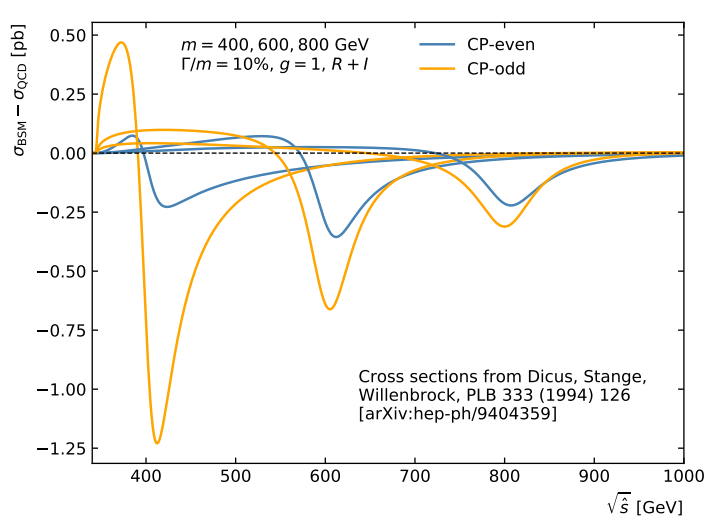

(a)

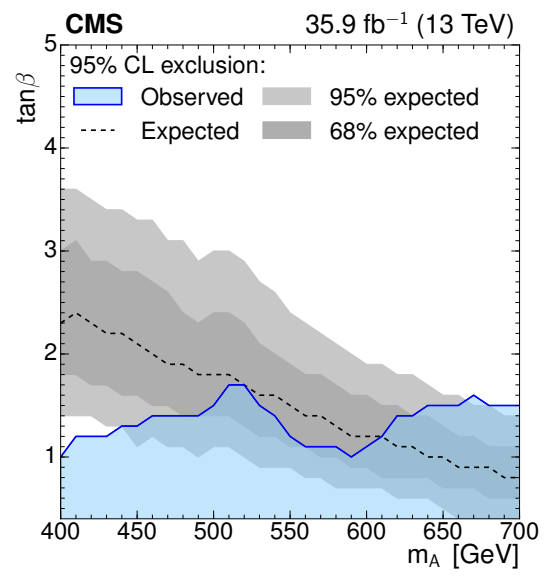

(b)

Figure 4. Results from a CMS search for a heavy neutral Higgs boson decaying to a pair of topquarks [94] showing: (a) examples of leading-order parton-level cross-sections after summing up the resonant and interference parts of the signal corresponding to neutral Higgs boson masses of 400, 600 and $800 \mathrm{GeV} ;(\mathbf{b})$ an interpretation of the search in the $\left(m_{A}, \tan \beta\right)$ plane of the hMSSM. Copyright 2021 CERN for the benefit of the CMS Collaboration. CC-BY-4.0 license.

\subsection{Searches for Heavy Neutral Higgs Bosons Decaying to Pairs of Electroweak Vector Bosons}

The SM-like Higgs boson with a mass of $125 \mathrm{GeV}$ was first discovered thanks to its bosonic decays ( $\gamma \gamma, Z Z$ and, to a lesser extent, $W W)$; hence, it is natural that such final states are also employed in order to search for additional neutral Higgs bosons, whenever the corresponding couplings are not suppressed. In the case of searches for $H \rightarrow W W, Z Z$, only final states involving at least one electron or muon are reviewed in this article.

Given the excellent diphoton invariant mass resolution, the $\gamma \gamma$ final state is a very valuable experimental signature to probe the existence of new resonances at the LHC. The corresponding ATLAS and CMS data analyses usually target a generic resonant state and use two benchmark signal models for the interpretation of the search results: a spinzero resonance arising from an extended Higgs sector and a spin-two graviton that is assumed to be the lightest Kaluza-Klein excitation in a Randall-Sundrum model [95,96]. The latest ATLAS search for diphoton resonances [97] uses the full Run-2 dataset and is optimised to target masses above $200 \mathrm{GeV}$. The main background comes from the nonresonant production of photon pairs and, to a lesser extent, from events containing a photon and a jet, as well as events with two jets, in which case, the jets are misidentified as photons. The final discriminant is the invariant mass $m_{\gamma \gamma}$ of the two photons. The resonant signal is modelled by convoluting the true line shape of the resonance with a double-sided Crystal Ball function that accounts for the detector resolution. Meanwhile, the continuum background is described by an analytical functional form, which is chosen by minimising the spurious signal (i.e., the bias obtained when fitting a background-only simulated and smoothed continuum background sample with a signal+background model). Figure 5a shows the $m_{\gamma \gamma}$ spectrum measured by ATLAS, with a background-only fit to the data and various signal line shapes overlaid. In the absence of any significant excess of data above the background predictions, exclusion limits are placed on the product of the fiducial cross-section and branching fraction, ranging from $12.5 \mathrm{fb}$ at $160 \mathrm{GeV}$ to $0.03 \mathrm{fb}$ at $2800 \mathrm{GeV}$, for a narrow spin-zero resonance, as shown in Figure $5 \mathrm{~b}$. Here, the fiducial volume is defined by requiring two generator-level photons with (i) $|\eta|<2.37$, (ii) a ratio $E_{T} / m_{\gamma \gamma}$ between the transverse energy and the diphoton invariant mass greater than, respectively, 0.3 and 0.25 for the leading and subleading photon, and (iii) particle isolation requirements mimicking those imposed at the detector level. 


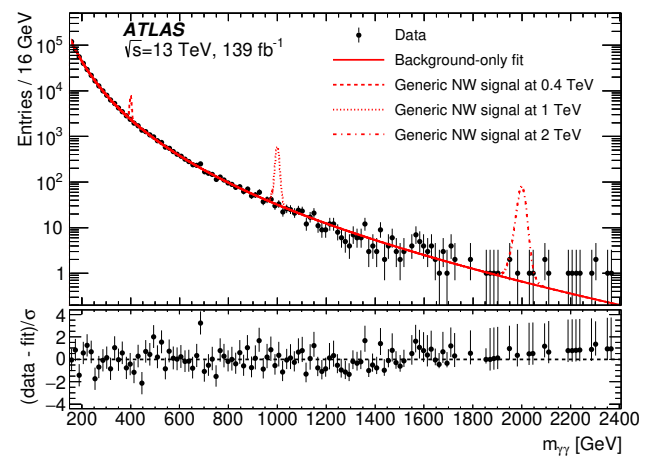

(a)

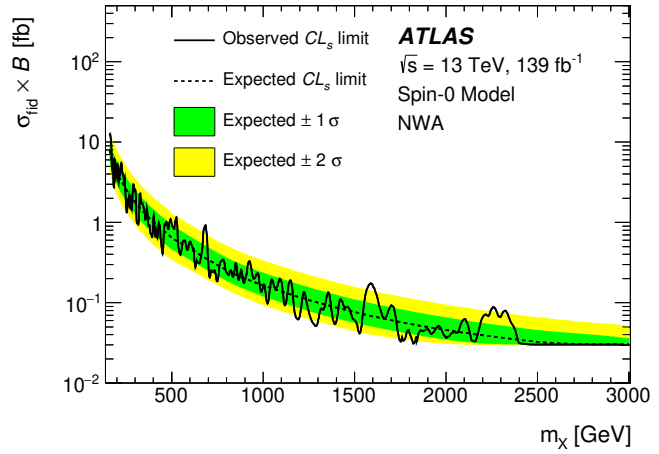

(b)

Figure 5. Selected results from the latest ATLAS search for spin-zero resonances decaying to two photons [97] showing in particular: (a) a background-only fit to the $m_{\gamma \gamma}$ spectrum, with signal shapes overlaid and normalised to 10-times the corresponding expected limit; (b) observed and expected 95\% CL limits on the fiducial cross-section times the branching fraction to two photons for a narrow spin-zero resonance, as a function of its mass. Copyright 2021 CERN for the benefit of the ATLAS Collaboration. CC-BY-4.0 license.

A similar search for a high-mass spin-zero resonance decaying to two photons has also been performed with the CMS experiment, and results based on a partial Run-2 dataset $\left(36 \mathrm{fb}^{-1}\right)$ were reported in [98]. In addition, CMS also searched for an additional Higgs boson decaying to $\gamma \gamma$ in the mass range $70-110 \mathrm{GeV}$ by using a combination of the Run-1 dataset at $8 \mathrm{TeV}$ (corresponding to an integrated luminosity of $19.7 \mathrm{fb}^{-1}$ ) and of $36 \mathrm{fb}^{-1}$ of Run-2 data at $13 \mathrm{TeV}$ [99]. A multi-variate classifier is used to discriminate signal events from the diphoton continuum background, and events are categorised according to the classifier score. In each category, the diphoton invariant mass is used as the final discriminant, as illustrated in Figure 6a, and analytical fit functions are employed to describe the background. However, in contrast with the searches performed above the SM-like Higgs boson mass, the $m_{\gamma \gamma}$ range of this analysis includes the $Z$ boson region. Hence, an additional background arises from Drell-Yan production of electron pairs, which may appear as two isolated photons through misidentification. Figure $6 \mathrm{~b}$ shows the exclusion limits set by CMS on the production cross-section times the branching fraction of an additional Higgs boson decaying to two photons. A small excess of data with a local (global) significance of $2.8 \sigma(1.3 \sigma)$ is reported at $95.3 \mathrm{GeV}$. 


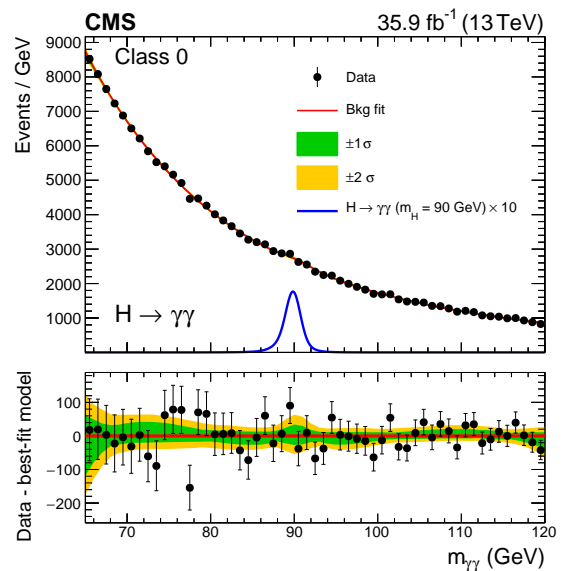

(a)

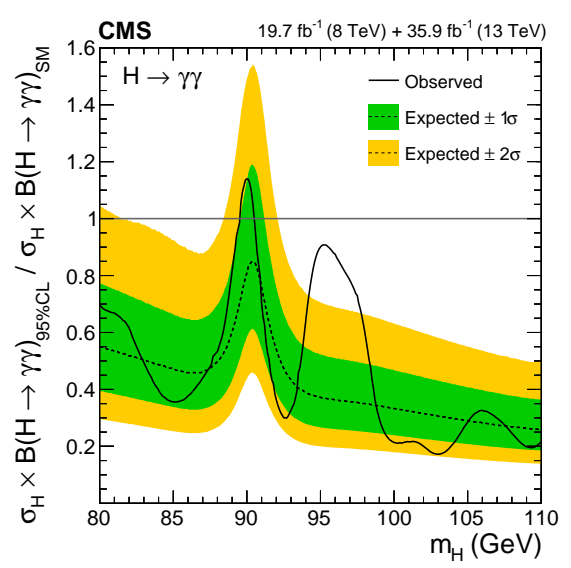

(b)

Figure 6. Selected results from the CMS search for spin-zero resonances decaying to two photons reported in [99] showing in particular: (a) a background-only fit to the $m_{\gamma \gamma}$ spectrum in the most sensitive event category of the $13 \mathrm{TeV}$ dataset, with an illustrative $90 \mathrm{GeV}$ signal shape overlaid; (b) observed and expected 95\% CL limits on the production cross-section times the branching fraction of an additional Higgs boson decaying to two photons, relative to the expected SM-like value, from a combined analysis of $8 \mathrm{TeV}$ and $13 \mathrm{TeV}$ data. Copyright $2021 \mathrm{CERN}$ for the benefit of the CMS Collaboration. CC-BY-4.0 license.

Unless theoretically suppressed (e.g., because of a vanishing coupling in the alignment limit of the 2HDM), the decay of a heavy neutral CP-even Higgs boson into a pair of vector bosons $(V V)$ offers a multitude of decay chains as experimental probes. The $V V$ search channels become particularly interesting in the Georgi-Machacek model, where there is no issue of suppression due to alignment, because the neutral Higgs bosons arise from the fiveplet or triplet states. Leaving fully hadronic final states aside, searches for $H \rightarrow W W / Z Z$ have been performed in ATLAS and CMS by using either fully leptonic ( $W W \rightarrow \ell v \ell^{\prime} v$, $\left.Z Z \rightarrow \ell \ell v v, Z Z \rightarrow \ell \ell \ell^{\prime} \ell^{\prime}\right)$ or semileptonic $(W W \rightarrow \ell v q q, Z Z \rightarrow \ell \ell q q, Z Z \rightarrow v v q q)$ signatures. Based on a partial Run-2 dataset with an integrated luminosity of $36 \mathrm{fb}^{-1}$, ATLAS has published four search results, considering separately fully leptonic ZZ decays [100], semileptonic ZZ decays [101], fully leptonic $W W$ decays [102] and semileptonic $W W$ decays [103]. A statistical combination of these searches, together with those performed in hadronic final states, is reported in [104]. Figure 7a,b shows the corresponding upper limits on the cross-section times the branching fraction for a generic narrow spin-zero resonance decaying to $W W$ or ZZ. With a similar dataset, CMS published two search results, one focusing on fully and semileptonic ZZ final states [105] and another one targeting fully and semileptonic WW final states [106]. Illustrative two-dimensional exclusion limits in the type-I and type-II $2 \mathrm{HDM}\left(m_{H}, \tan \beta\right)$ planes are shown in Figure $7 \mathrm{c}, \mathrm{d}$.

In all searches above, several benchmark signal models are considered for the interpretation of the results: in addition to spin-zero resonances arising from an extended Higgs sector, heavy vector bosons (spin-one) and gravitons (spin-two) are also searched for in the $V V$ final states listed above. Given the variety of signal models probed by the analyses, several production modes must be targeted, including gluon-gluon fusion and VBF (which are particularly relevant for spin-zero interpretations), as well as quark-antiquark annihilation. Furthermore, in the case of semileptonic decays of $W W$ or $Z Z$ resonances, the jets arising from the hadronic decay of one of the bosons tend to overlap in the detector if the mass of the resonance is large, typically above $1 \mathrm{TeV}$. Hence, in addition to event categories that target the various production modes, "resolved" and "merged" topologies are also considered by the analyses, in which respectively two small-radius jets or one large-radius jet are used to reconstruct the $W / Z \rightarrow q q$ decay. However, the merged topologies are usually of limited interest for interpretations in the context of extended Higgs sectors, as 
the natural width of the $\mathrm{CP}$-even $H$ boson becomes too large for such searches to be really meaningful at high masses.

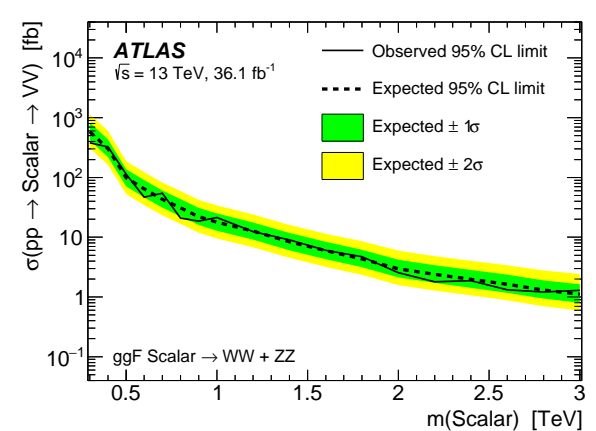

(a)

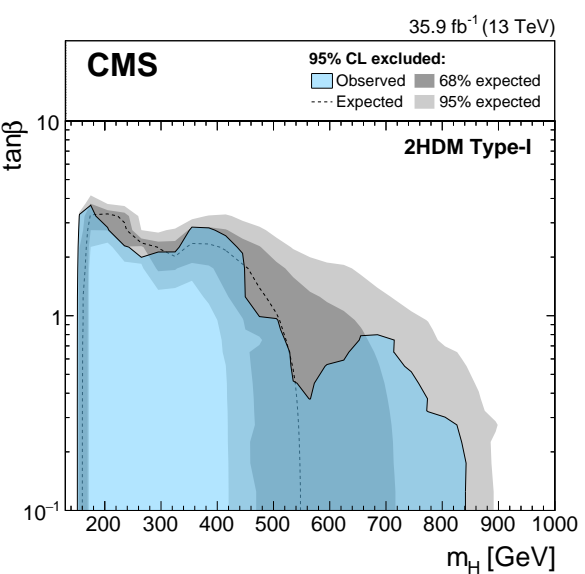

(c)

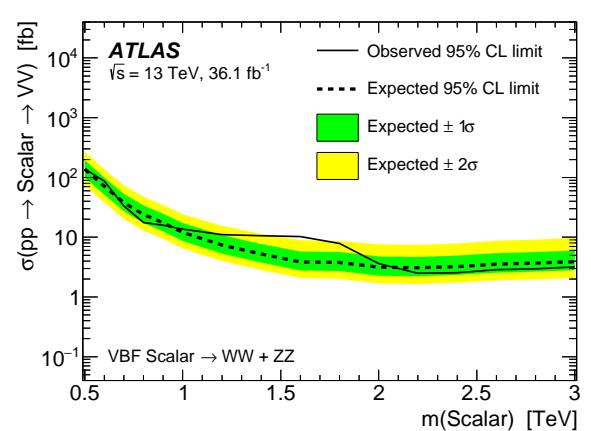

(b)

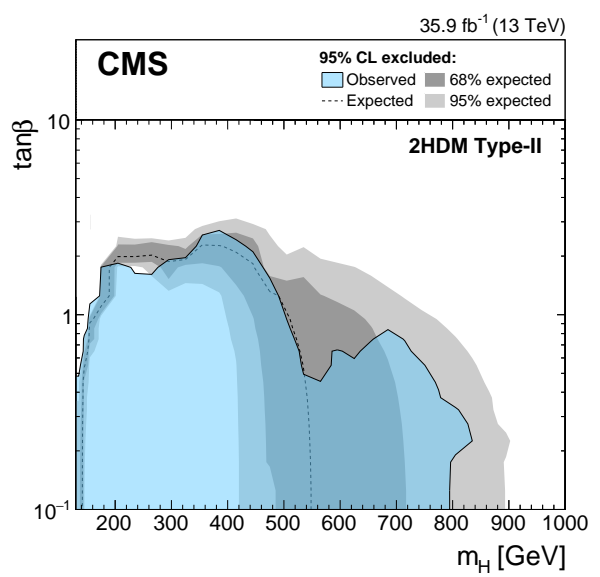

(d)

Figure 7. Top: expected and observed 95\% CL limits set by ATLAS on the cross-section times the branching fraction to $W W$ or $Z Z$ for a generic scalar particle produced via (a) gluon-gluon fusion or (b) VBF, as reported in [104]. Bottom: two-dimensional constraints in the $\left(m_{H}, \tan \beta\right)$ plane of (c) the type-I 2HDM and (d) the type-II 2HDM, assuming $m_{A}=m_{H}$ and $\cos (\beta-\alpha)=0.1$, as reported by CMS in their search for $H \rightarrow W W$ [106]. Copyright 2021 CERN for the benefit of the ATLAS Collaboration (a,b) and CMS Collaboration (c,d). CC-BY-4.0 license.

More recently, ATLAS published results from an updated search for $Z Z$ resonances in fully leptonic final states ( $\ell \ell v v$ and $\left.\ell \ell \ell^{\prime} \ell^{\prime}\right)$, based on the full Run-2 dataset at $13 \mathrm{TeV}$ [107]. In the data analysis, events are classified into categories enriched in either gluon-gluon fusion or VBF production, for both final states. In events with four reconstructed leptons (electrons and/or muons), their invariant mass $m_{4 \ell}$ is used as the final discriminant, while events containing a lepton pair with a transverse momentum $\vec{p}_{T}^{\ell \ell}$ and missing transverse momentum $\vec{p}_{T}^{m i s s}$ from escaping neutrinos use a transverse mass discriminant, defined as:

$$
m_{T}^{Z}=\sqrt{\left[\sqrt{m_{Z}^{2}+\left(p_{T}^{\ell \ell}\right)^{2}}+\sqrt{m_{Z}^{2}+\left(p_{T}^{m i s s}\right)^{2}}\right]^{2}-\left|\vec{p}_{T}^{\ell \ell}+\vec{p}_{T}^{m i s s}\right|^{2}},
$$

where $m_{Z}$ is the mass of the $Z$ boson. Illustrative distributions of these two discriminants are shown in Figure 8a,b.

In the absence of any excess of data with respect to the SM backgrounds, exclusion limits are set on the production rate of various signal models. With the assumption that the new CP-even heavy Higgs boson is narrow, the upper limits are also interpreted as exclusion contours in the various types of 2HDM and in several MSSM benchmark 
scenarios, as illustrated in Figure 9. The results are also interpreted in models with nonnegligible natural widths of the scalar resonance (up to $15 \%$ of its mass), but only gluongluon fusion is considered in that case. Given that the $g g \rightarrow \mathrm{ZZ}$ continuum background and the $g g$-initiated production mode of both a heavy neutral Higgs boson and the SM-like $h$ share the same initial and final states, interference effects must be taken into account when considering broad scalar resonances decaying into ZZ.

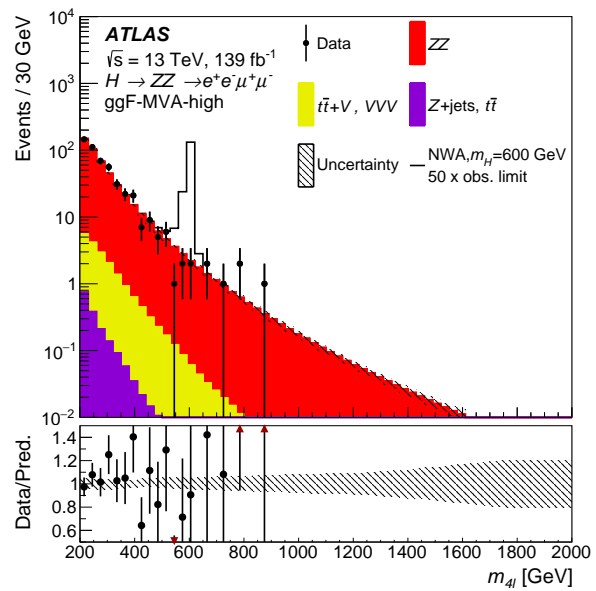

(a)

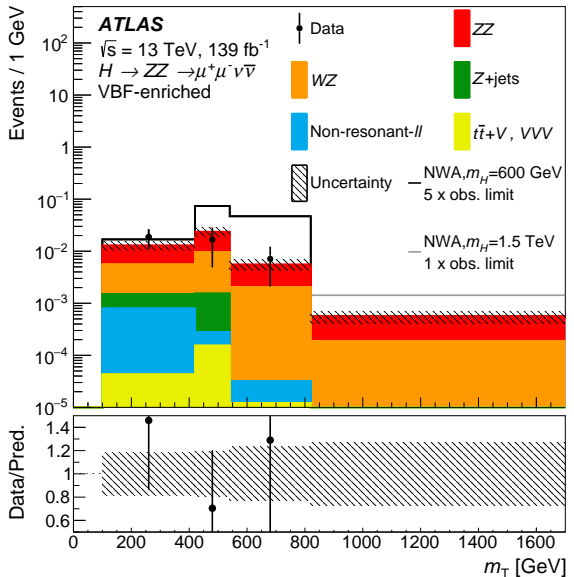

(b)

Figure 8. Example distributions of (a) $m_{4 \ell}$ for $е e \mu \mu$ events enriched in gluon-gluon fusion and (b) $m_{T}^{Z}$ in VBF-enriched $\mu \mu v v$ events, from a background-only fit to data and with various narrow-width heavy neutral Higgs boson signals overlaid [107]. Copyright 2021 CERN for the benefit of the ATLAS Collaboration. CC-BY-4.0 license.

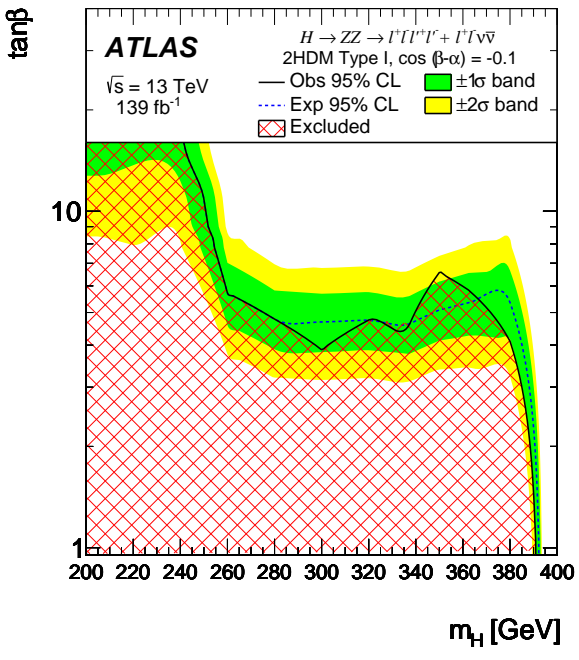

(a)

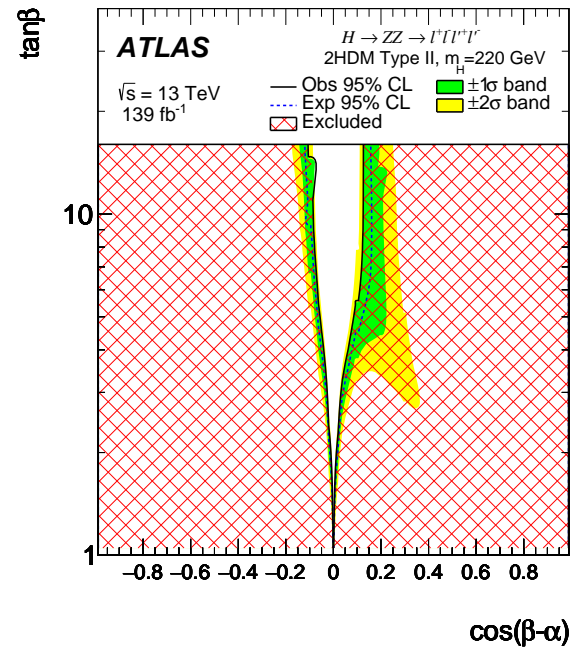

(b)

Figure 9. Example of two-dimensional constraints in (a) the $\left(m_{H}, \tan \beta\right)$ plane of the type-I 2HDM, assuming $m_{A}=m_{H}$ and $\cos (\beta-\alpha)=-0.1$ and $(\mathbf{b})$ the $(\cos (\beta-\alpha), \tan \beta)$ plane of the type-II 2HDM, assuming $m_{A}=m_{H}=220 \mathrm{GeV}$, as reported by ATLAS in [107]. Copyright 2021 CERN for the benefit of the ATLAS Collaboration. CC-BY-4.0 license.

\subsection{Search for $A \rightarrow \mathrm{ZH} / \mathrm{Zh}$}

The decay of a heavy pseudoscalar resonance, $A$, to a pair of vector bosons is suppressed in all $2 \mathrm{HDM}$ types. Therefore, the most favourable search channel for $A$ is through its decay to a $Z$ boson and a CP-even Higgs boson, $A \rightarrow Z H / h$. The decay $A \rightarrow Z H$ is most sensitive close to the weak decoupling limit, where the coupling $A Z h$ vanishes. In 
this section, searches for $A \rightarrow Z H$ are reported in somewhat more detail, and a shorter discussion of $A \rightarrow Z h$ will then follow.

The scenario in which the heavy neutral Higgs bosons $A$ and $H$ have different masses (where $H$ is a $\mathrm{CP}$-even Higgs boson other than that with a mass of $125 \mathrm{GeV}$ ) opens new parts of the 2HDM parameter space. Furthermore, it is motivated by electroweak baryogenesis scenarios, in which the mass hierarchy $m_{A}>m_{H}$ is preferred for a strong first-order phase transition in the early universe. In the relevant regions of the 2HDM parameter space, the production of a heavy neutral Higgs boson subsequently decaying to a $Z$ boson and another heavy Higgs boson occurs both via gluon-gluon fusion and in association with $b$ quarks at the LHC. Both ATLAS and CMS have searched for this experimental signature by exploiting the $\ell \ell b b$ final state arising from the decays $Z \rightarrow e e / \mu \mu$ and $A / H \rightarrow b b[88,108]$. While the ATLAS search for $A \rightarrow Z H$ [88] uses the full Run-2 dataset and targets both production modes by using " $2 b$-jet" and " $\geq 3 b$-jet" event categories, the CMS search of [108] uses only $36 \mathrm{fb}^{-1}$ of $13 \mathrm{TeV}$ data and focuses solely on the production via gluongluon fusion. In addition, the ATLAS publication also includes a search result in the $Z(\rightarrow e e / \mu \mu) H(\rightarrow W W)$ decay mode, whereas a previous CMS result [109] also considered $Z(\rightarrow e e / \mu \mu) H(\rightarrow \tau \tau)$ events.

Here, the description of the data analyses will focus mostly on the $\ell \ell b b$ final state, which is common to both the ATLAS and CMS searches. The general strategy is to first reconstruct a $Z \rightarrow e e / \mu \mu$ candidate from two electrons or muons that have an invariant mass compatible with $m_{Z}$. Then, the ATLAS search requires the mass of the two $b$-jets that form the Higgs boson candidate to lie within a window that has a width compatible with the resolution for the considered $H$ boson mass hypothesis. For events found in this window, the reconstructed invariant mass of the $\ell \ell b b$ system is the discriminating variable that is eventually employed to obtain the final result, as shown in Figure 10a. A similar selection and strategy is also used for the $\ell \ell W W$ final state, where both $W$ bosons are assumed to decay hadronically. Hence, the invariant mass of the Higgs boson candidate is reconstructed using four jets; see Figure $10 \mathrm{~b}$ for an illustration. The ATLAS search only covers the $2 \mathrm{HDM}$ parameter space relevant for the $A \rightarrow \mathrm{ZH}$ decay, leaving out the possibility of $H \rightarrow Z A$ decay chains, because the natural width of $H$ is small enough for the $\mathrm{CP}$-even Higgs boson to be considered narrow in the 2HDM parameter space where the search tends to be sensitive, whereas this is not the case for the $A$ boson. Hence, the signal modelling considered in the ATLAS search allows for the production of a pseudoscalar boson with widths up to $20 \%$ of the $A$ mass, which is larger than the experimental resolution, but no such provision is made for the $H$ boson at the end of the decay chain. On the other hand, the CMS search does include $H \rightarrow Z A$ decays. The analysis is similar to that employed in the ATLAS search, with the main difference being that CMS uses a two-dimensional cut in the plane defined by the reconstructed masses of the $A$ and $H$ bosons. As a result, the shape of the signal region is an ellipse, and the $\left(m_{j j}, m_{\ell \ell j j}\right)$ plane is therefore divided in elliptical concentric bands, as illustrated by Figure 11a, for which a one-dimensional representative distribution eventually enters the statistical analysis used for the signal extraction; see Figure 11b. The main backgrounds in the ATLAS and CMS searches are the productions of a $Z$ boson in association with heavy-flavour quarks and of $t t$ pairs. 


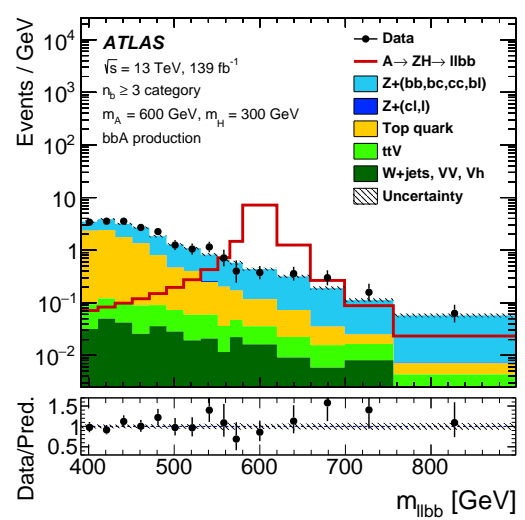

(a)

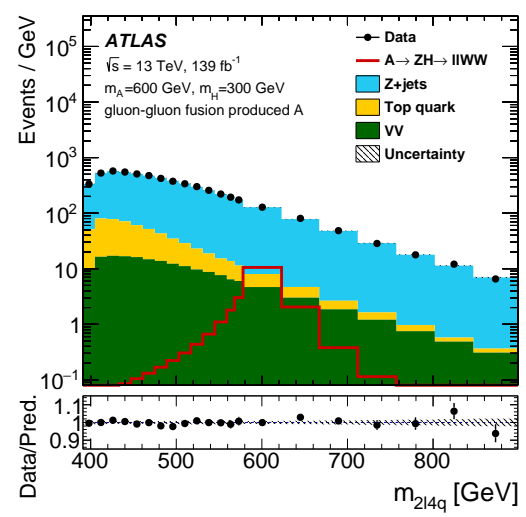

(b)

Figure 10. Distributions of (a) $m_{\ell \ell b b}$ in a signal region with at least $3 b$-jets after selecting events falling in the adequate $m_{b b}$ mass window and of (b) $m_{2 \ell 4 q}$ after selecting events falling in the adequate $m_{4 q}$ mass window. The signal mass hypotheses are $m_{A}=600 \mathrm{GeV}$ and $m_{H}=300 \mathrm{GeV}$. Both plots are from the ATLAS search for $A \rightarrow Z H$ [88]. Copyright 2021 CERN for the benefit of the ATLAS Collaboration. CC-BY-4.0 license.

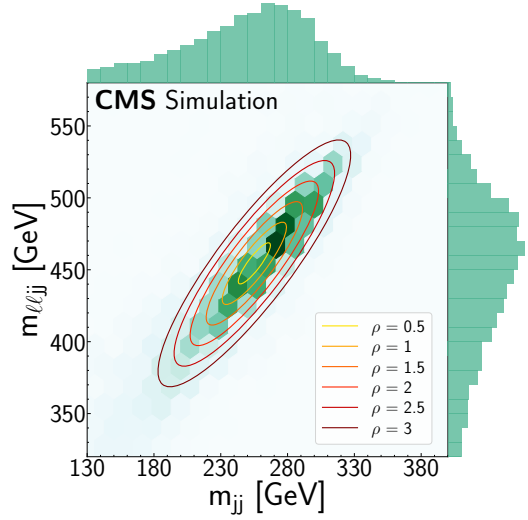

(a)

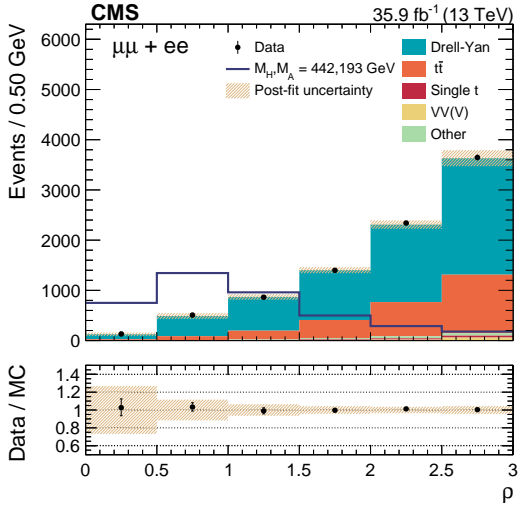

(b)

Figure 11. Examples of event selections used in the CMS search for $A \rightarrow Z H$ [108] showing: (a) a two-dimensional set of elliptical regions used to define the signal region for a parameter space point with $m_{A}=500 \mathrm{GeV}$ and $m_{H}=300 \mathrm{GeV}$, where the variable $\rho$ is used to parameterise concentric elliptical regions in which events enter the statistical analysis in a later stage; $(\mathbf{b})$ the final discriminant in the signal region corresponding to a signal hypothesis with $m_{A}=193 \mathrm{GeV}$ and $m_{H}=442 \mathrm{GeV}$. Copyright 2021 CERN for the benefit of the CMS Collaboration. CC-BY-4.0 license.

In the absence of any significant excess in the data, the search results are presented as model-independent upper limits on the cross-section for a grid of $m_{A}$ and $m_{H}$ values. For instance, Figure 12a shows ATLAS search results for $A \rightarrow Z H$ produced in gluon-gluon fusion and based on the $\ell \ell b b$ final state. Such exclusion limits are then interpreted in the context of the 2HDM. Again in the case of the $\ell \ell b b$ final state, ATLAS shows such results in the $\left(m_{H}, m_{A}\right)$ plane, for various $\tan \beta$ values and in the weak decoupling limit $\cos (\beta-\alpha)=0$, as illustrated in Figure 12b. For the ATLAS search targeting the $\ell \ell W W$ decay chain, the $\left(\cos (\beta-\alpha), m_{A}\right)$ plane is chosen instead, and results are shown for various values of $m_{H}$ and $\tan \beta$; see, e.g., Figure 12c. In the interpretations of their search results in the $\ell \ell b b$ final state, CMS includes departures from the weak decoupling limit and also puts some constraints in the plane $(\cos (\beta-\alpha), \tan \beta)$ for various combinations of $m_{A}$ and $m_{H}$, as illustrated by Figure 13. 


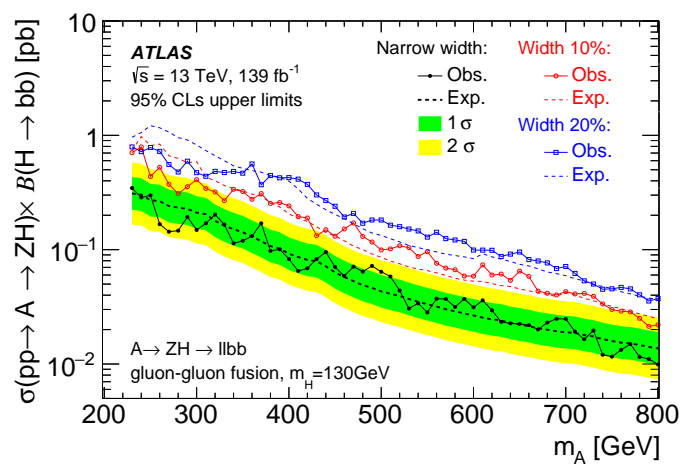

(a)

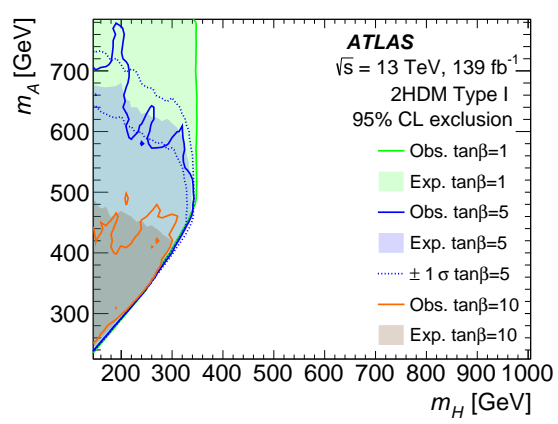

(b)

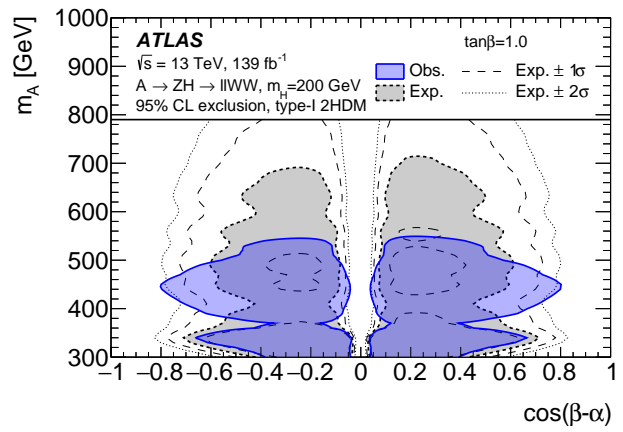

(c)

Figure 12. Examples of exclusion limits from the ATLAS $A \rightarrow Z H$ search of [88] showing: (a) 95\% CL limits on the cross-section times the branching fraction of a neutral Higgs boson $A \rightarrow Z H(b b)$, with various widths, produced via gluon-gluon fusion and searched for in the $\ell \ell b b$ final state; (b) two-dimensional constraints in the $\left(m_{H}, m_{A}\right)$ plane of the type-I $2 \mathrm{HDM}$, for various $\tan \beta$ values and in the weak decoupling limit ( $\ell b b$ final state); (c) two-dimensional constraints in the $\left(\cos (\beta-\alpha), m_{A}\right)$ plane of the type-I 2HDM, for a given combination of $m_{H}$ and $\tan \beta$ ( $\ell \ell W$ final state). Copyright 2021 CERN for the benefit of the ATLAS Collaboration. CC-BY-4.0 license.

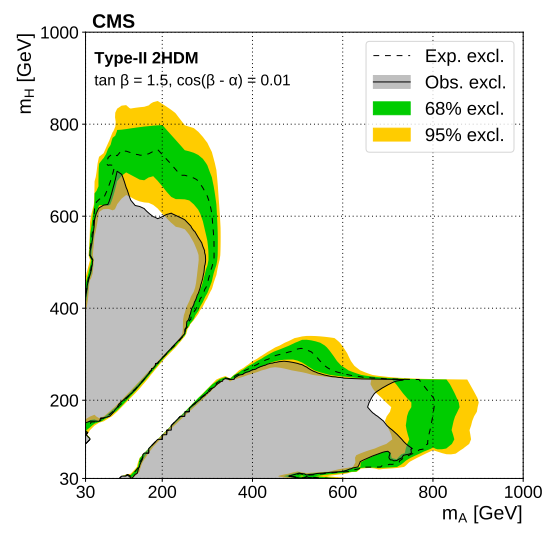

(a)

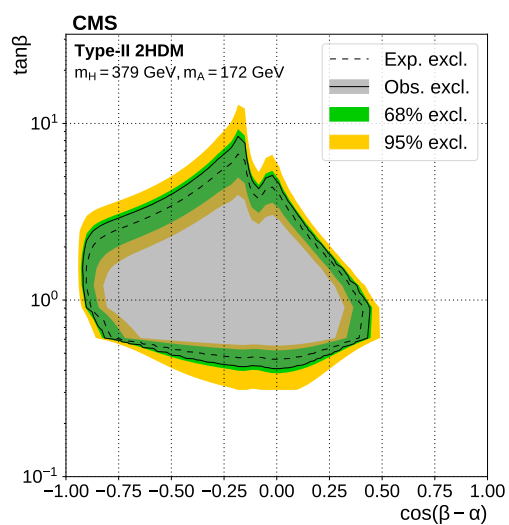

(b)

Figure 13. Examples of exclusion limits from the CMS search for a neutral Higgs boson decaying to a $Z$ boson and another heavy Higgs boson in the $\ell \ell b b$ final state [108] showing: (a) two-dimensional constraints in the $\left(m_{A}, m_{H}\right)$ plane of the type-II $2 \mathrm{HDM}$, for a given combination of $\tan \beta$ and $\cos (\beta-\alpha)$ values; (b) two-dimensional constraints in the $(\cos (\beta-\alpha), \tan \beta)$ plane of the type-II 2HDM, for a given combination of $m_{H}$ and $m_{A}$. Copyright 2021 CERN for the benefit of the CMS Collaboration. CC-BY-4.0 license. 
The decay $A \rightarrow Z h$, where $h$ is the already discovered SM-like Higgs boson, is usually searched for in the $\ell \ell b b$ channel in which $Z \rightarrow e e / \mu \mu$ and $h \rightarrow b b$. The latest corresponding search results from ATLAS and CMS are detailed in [110,111]. The ATLAS publication also includes a complementary search channel, in which the decays $Z \rightarrow v v$ and $h \rightarrow b b$ are considered. In addition, CMS has a dedicated publication that employs the decays $Z \rightarrow e e / \mu \mu$ and $h \rightarrow \tau \tau$ [112]. These searches can also be interpreted in the context of models with heavy vector triplets [113], which are in general easier than extended Higgs sectors, because they involve heavy resonances with smaller natural widths. In the context of $2 \mathrm{HDM}$ interpretations, the most sensitive final state is generally $\ell \ell b b$. However, in some cases, such as the lepton-specific $2 \mathrm{HDM}$, the $\ell \ell \tau \tau$ final state provides more sensitivity. The interpretation of these searches in the $2 \mathrm{HDM}$ is usually performed in the $(\cos (\beta-\alpha), \tan \beta)$ plane, as illustrated in Figure 14a from [111]. Because the search for $A \rightarrow \mathrm{Zh}$ is also relevant in the low $\tan \beta$ region of the MSSM, it is also commonly interpreted in some MSSM benchmark scenarios; see, for instance, [114] and Figure 14b from [112].

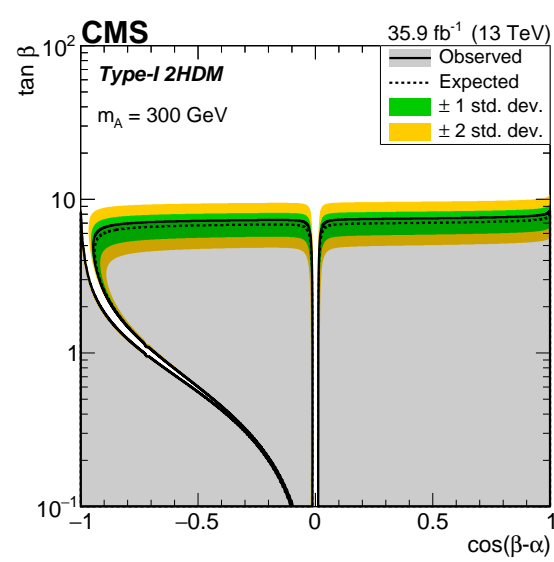

(a)

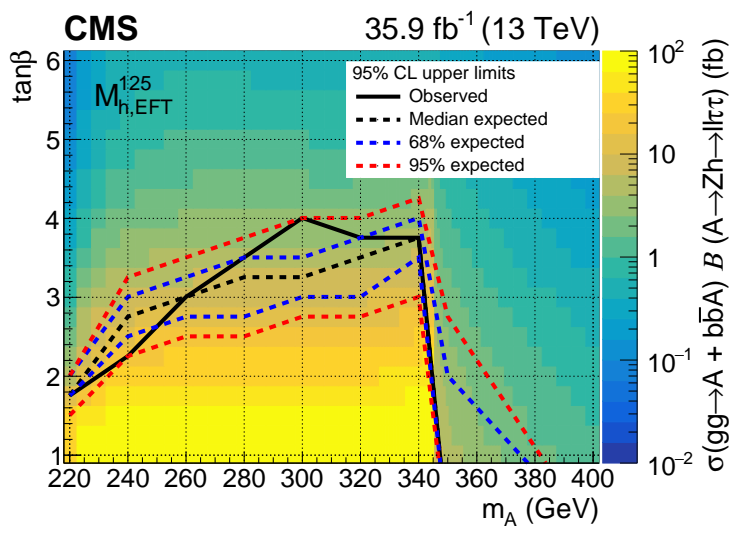

(b)

Figure 14. Examples of interpretations of CMS $A \rightarrow Z h$ search results showing: (a) constraints in the type-I $2 \mathrm{HDM}(\cos (\beta-\alpha), \tan \beta)$ plane, based on the $\ell \ell b b$ final state [111]; (b) constraints in the $\left(m_{A}, \tan \beta\right)$ plane of an MSSM benchmark scenario, based on the $\ell \ell \tau \tau$ final state [112]. Copyright 2021 CERN for the benefit of the CMS Collaboration. CC-BY-4.0 license.

\subsection{Searches for Light Higgs Boson States}

Light pseudoscalar Higgs boson states with a mass smaller than $m_{h} / 2$ are predicted in several extensions of the Higgs sector, and they are even favoured in some theories such as the NMSSM. Meanwhile, the Higgs boson that has been discovered at the LHC in 2012 may still decay in ways that have not been observed yet. Indeed, measurements of the $125 \mathrm{GeV}$ Higgs boson production and decays, such as those recently summarised in [21], still leave ample room for invisible and/or undetected decays. In turn, this motivates searches for light pseudoscalar Higgs bosons in some exotic decays of the $125 \mathrm{GeV}$ Higgs boson.

In principle, model-independent direct searches for the production of light pseudoscalars are possible, and some were even performed at the start of the LHC operation. For instance, both ATLAS and CMS have searched for inclusive production of $a \rightarrow \mu \mu[115,116]$. However, these data analyses suffer from large backgrounds, and triggering on relatively low- $p_{T}$ muons is challenging. Besides, other decay modes of the pseudoscalar particle $a$, such as $a \rightarrow b b$, are even more difficult to search for. On the other hand, in most extensions of the Higgs sector, there can be a sizeable coupling between these new pseudoscalar particles and the heavier Higgs bosons in large parts of the parameter space; hence, searches for the light pseudoscalar Higgs bosons are usually performed in exotic decays of the $125 \mathrm{GeV}$ Higgs boson, $h \rightarrow a a$. These search channels are categorised according to the decay modes of the $a a$ pair. A popular decay chain, which has been searched for with several datasets at the LHC, is $h \rightarrow a a \rightarrow b b \mu \mu$. The most recent results are reported in $[117,118]$. Both ATLAS and CMS searches for $h \rightarrow a a \rightarrow b b \mu \mu$ select events containing two muons and 
two $b$-jets using single- or dimuon triggers. The muons and $b$-jets in the event are then required to be compatible with the event kinematics. For this purpose, ATLAS employs a kinematic likelihood fit that exploits the equal invariant masses of the $b b$ and $\mu \mu$ systems in the $h \rightarrow$ a a decay, while CMS requires that events satisfy $\chi^{2}=\chi_{b b}^{2}+\chi_{h}^{2}<5$, where:

$$
\chi_{b b}=\frac{\left(m_{b b}-m_{\mu \mu}\right)}{\sigma_{b b}} \text { and } \chi_{h}=\frac{\left(m_{\mu \mu b b}-m_{h}\right)}{\sigma_{h}} .
$$

Here, $m_{b b}$ and $m_{\mu \mu}$ are the invariant masses of the two $b$-jets and the two muons, respectively, $m_{h}=125 \mathrm{GeV}$ and $\sigma_{h}, \sigma_{b b}$ are the experimental mass resolutions of the Higgs boson and the $b b$ system, respectively. The main background sources are topquark production and Drell-Yan processes. In CMS, the sensitivity is further improved by an event categorisation based on the $b$-tagging discriminator, and the backgrounds are modelled with analytical functions fitted directly to the data. In ATLAS, a boosted decision tree (BDT) classifier is used to further discriminate between the signal and backgrounds. While simulation is employed to estimate the shape of the top-quark backgrounds, DrellYan processes are estimated by taking data templates in a control region with zero $b$-jets and subsequently applying a reweighting to account for kinematical differences between samples dominated by jets from light quarks or gluons and samples enriched in $b$-jets. In both experiments, the final result is extracted from the resulting $m_{\mu \mu}$ distributions, and exclusion limits are set for various hypothetical values of $m_{a}$, as illustrated in Figure 15 for the ATLAS search based on the full Run-2 dataset [117].

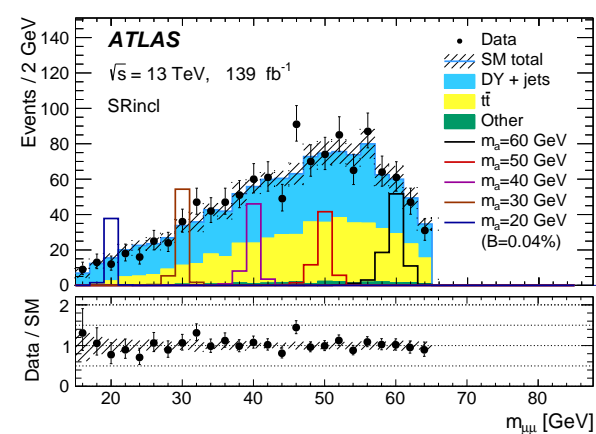

(a)

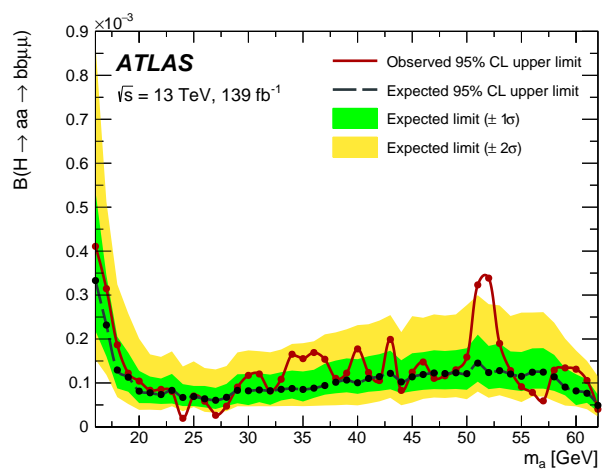

(b)

Figure 15. Examples of results from the ATLAS search for $h \rightarrow a a \rightarrow b b \mu \mu$ [117] showing: (a) the dimuon mass distribution prior to applying any BDT requirement; (b) 95\% CL limits on the branching fraction of a $125 \mathrm{GeV}$ Higgs boson decaying via $a a \rightarrow b b \mu \mu$, including the BDT selection, as a function of the signal mass hypothesis. Copyright 2021 CERN for the benefit of the ATLAS Collaboration. CC-BY-4.0 license.

Such upper limits can be interpreted in the $2 \mathrm{HDM}+\mathrm{S}$ extension of the SM, as, e.g., in Figure 16 extracted from the CMS search result based on a partial Run-2 dataset [118]. The strategies used in interpreting such searches in this model are discussed in detail in $[119,120]$. In particular, fixing the branching fraction of $h \rightarrow a a$ to a given value implies that the branching fraction of $a \rightarrow \mu \mu / b b$ depends only on the mass $m_{a}$ in the type-I $2 \mathrm{HDM}+\mathrm{S}$ (all Yukawa couplings are identical for $h$ ) or on one more parameter (e.g., $\tan \beta$ ) otherwise, for instance in the lepton-specific $2 \mathrm{HDM}+\mathrm{S}$ (mentioned as type-III in Figure 16). With this reduction of parameters, it is possible to show constraints as a function of $m_{a}$ or in the plane $\left(m_{a}, \tan \beta\right)$, depending on the choice of arrangement for the 2HDM Yukawa couplings. Finally, one further advantage of these benchmarks is that they can be used for the comparison of different final states on the same footing. 


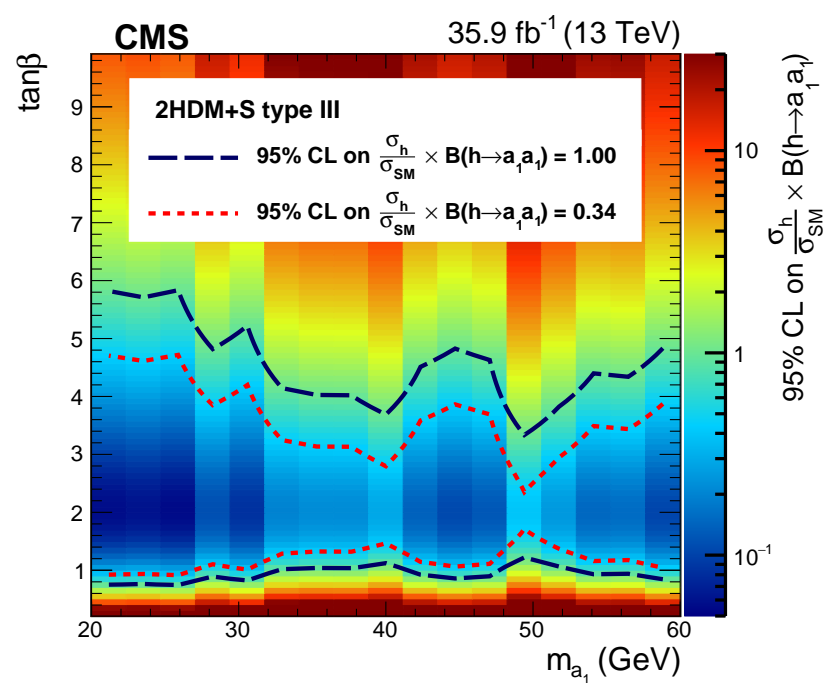

Figure 16. One of the interpretations of the upper limits set by CMS on the production cross-section of $h \rightarrow a a \rightarrow b b \mu \mu$ in a model with two Higgs doublets and a singlet, extracted from [118]. Copyright 2021 CERN for the benefit of the CMS Collaboration. CC-BY-4.0 license.

Indeed, besides $h \rightarrow a a \rightarrow b b \mu \mu$, several other decay channels have been searched for in ATLAS and CMS, such as $h \rightarrow a a \rightarrow 4 \mu$ [121-123], $h \rightarrow a a \rightarrow \mu \mu \tau \tau$ [124-126], $h \rightarrow a a \rightarrow b b \tau \tau$ [127], $h \rightarrow a a \rightarrow 4 \tau$ [123], $h \rightarrow a a \rightarrow 4 b$ [128], $h \rightarrow a a \rightarrow 4 \gamma$ [129,130], $h \rightarrow a a \rightarrow \gamma \gamma g g$ [131]. ATLAS and CMS regularly provide summary plots regarding the interpretations of such searches in the $2 \mathrm{HDM}+\mathrm{S}$ scenario. Figure 17 shows a selection of some of the most recent interpretations, with more summary plots being available in $[132,133]$.

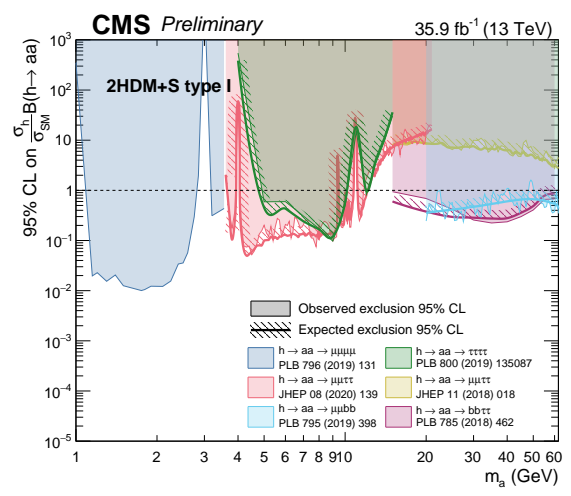

(a)

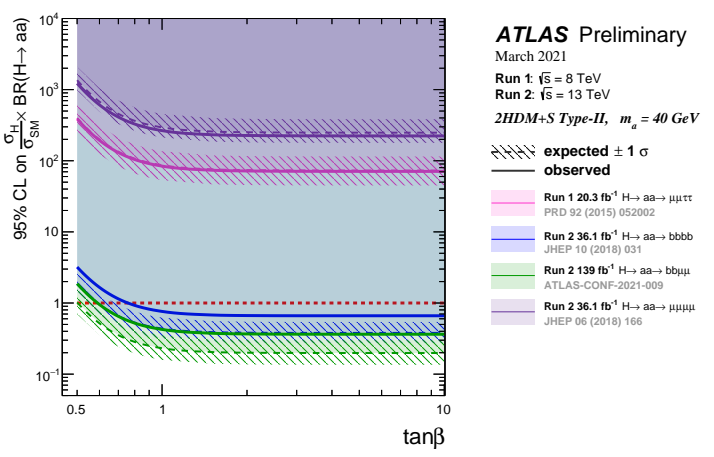

(b)

Figure 17. Examples of 2HDM+S interpretations of ATLAS and CMS search results for $h \rightarrow a a$ showing $95 \%$ CL limits on $\frac{\sigma_{h}}{\sigma_{h, S M}} \times B(h \rightarrow a a)$ in (a) the $2 \mathrm{HDM}+\mathrm{S}$ type-I scenario for different $m_{a}$ values, as reported by CMS in [132]; (b) the $2 \mathrm{HDM}+\mathrm{S}$ type-II scenario for different $\tan \beta$ values, at a fixed $m_{a}$ value of $40 \mathrm{GeV}$, as reported by ATLAS in [133]. Copyright 2021 CERN for the benefit of the CMS Collaboration (a) and ATLAS Collaboration (b). CC-BY-4.0 license.

\section{Searches for Charged Higgs Bosons}

Charged Higgs bosons are predicted in any extended Higgs sector that contains more than just additional weak isospin singlets. Singly charged Higgs bosons $H^{ \pm}$arise from scalar doublets, while doubly charged Higgs bosons $H^{ \pm \pm}$are also predicted whenever scalar triplets are introduced. Since the SM does not contain any physical charged scalar state, the observation of such particles would clearly point to new physics. The ATLAS and CMS Collaborations have an extensive search program for singly and doubly charged Higgs bosons. This section presents a review of the search results obtained so far with the partial or full Run-2 dataset at $\sqrt{s}=13 \mathrm{TeV}$. 


\subsection{Search Results for Singly Charged Higgs Bosons in Fermionic Decay Channels}

The two flagship $H^{ \pm}$searches at the LHC have historically been conducted in the $\tau v$ and $t b$ decay channels, because they allow to cover complementary regions of the MSSM parameter space. While the decay $H^{ \pm} \rightarrow t b$ is only kinematically allowed for charged Higgs boson masses $m_{H^{ \pm}}>m_{t}+m_{b}$, the $H^{ \pm} \rightarrow \tau v$ search channel allows to probe a broader mass range, typically from nearly the $W$-boson mass $m_{W}$ up to the TeV scale. Given the large coupling between charged Higgs bosons and heavy fermions, especially in $2 \mathrm{HDMs}$, such searches are performed in production modes involving the presence of additional heavy quarks, i.e., $p p \rightarrow t b H^{ \pm}$, leading to complex final states combining several detector signatures. Among the fermionic search channels, the decays of charged Higgs bosons to light quarks, $H^{ \pm} \rightarrow c s / c b$, provide an additional probe of the mass range below $m_{t}$. They also serve as experimental signatures of extended Higgs sectors beyond the 2HDM.

\subsection{1. $H^{ \pm} \rightarrow \tau v$}

The $H^{ \pm} \rightarrow \tau v$ search channel allows to probe charged Higgs boson masses below, close to and above the top-quark mass. The corresponding leading-order Feynman diagrams are shown in Figure 18. In a type-II 2HDM, $H^{ \pm} \rightarrow \tau v$ is the dominant decay when $H^{ \pm}$is lighter than the top-quark, and it has a sizeable branching fraction at large $\tan \beta$ when $H^{ \pm}$is heavier than the top-quark. The ATLAS and CMS Collaborations have searched for $H^{ \pm} \rightarrow \tau v$ in their partial Run-2 datasets collected in 2015-2016, as reported in $[134,135]$, respectively.

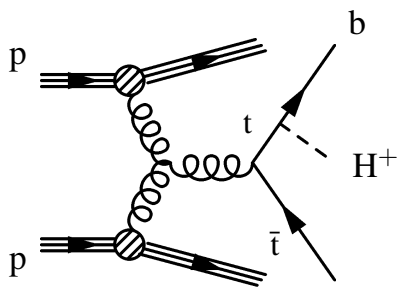

(a)

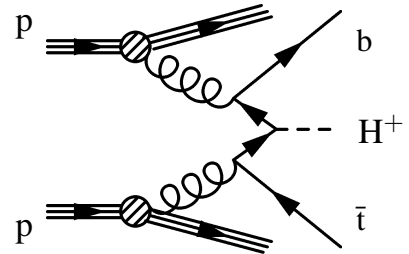

(b)

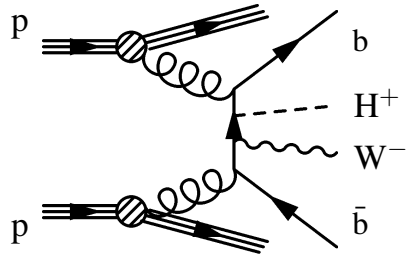

(c)

Figure 18. Leading-order Feynman diagrams for charged Higgs boson production via (a) doubleresonant top-quark production that dominates when $H^{ \pm}$is lighter than the top-quark, (b) singleresonant top-quark production that dominates when $H^{ \pm}$is heavier than the top-quark. In the intermediate region where $m_{H^{ \pm}} \simeq m_{t}$, both production modes contribute, together with (c) nonresonant top-quark production. All diagrams are from [135]. Copyright 2021 CERN for the benefit of the CMS Collaboration. CC-BY-4.0 license.

In the ATLAS search, only hadronic decays of the $\tau$-lepton arising from $H^{ \pm} \rightarrow \tau v$ are considered and two final states are used: $\tau+$ jets (where the associated top-quark decays fully hadronically) and $\tau+$ lepton (where the $W$ boson coming from the associated top-quark decays into an electron or a muon). The CMS search considers an additional leptonic final state without any hadronically decaying $\tau$-lepton. The $\tau+$ jets final state provides the bulk of the sensitivity in the search for $H^{ \pm}$above the top-quark mass. In that case, the neutrino from the $H^{ \pm} \rightarrow \tau v$ decay yields a large amount of missing transverse energy $\left(E_{T}^{\text {miss }}\right)$ on which events are triggered. The $E_{T}^{\text {miss }}$ trigger threshold is $90 \mathrm{GeV}$ in CMS and varies between 70 and $110 \mathrm{GeV}$ in ATLAS, depending on the data-taking period and thereby accounting for different pile-up conditions. Such high $E_{T}^{\text {miss }}$ thresholds yield a small signal efficiency in the case of light $H^{ \pm} \rightarrow \tau v$ events. Therefore, in order to also ensure sensitivity to light $H^{ \pm}$produced in top-quark decays, the ATLAS and CMS searches additionally target $\tau$-lepton decays into an electron or muon, in turn allowing to use single-lepton triggers.

In both searches, the background arising from the misidentification of a quark- or gluon-initiated jet as a hadronic $\tau$ object is sizeable. The estimation of this background starts from a region enriched in misidentified $\tau$ candidates, which is obtained by only 
inverting the $\tau$ identification criteria with respect to the nominal event selection (signal region). After subtraction of simulated events with a true $\tau$ candidate, fake factors are applied in order to predict the fake- $\tau$ background in the signal region. Such fake factors are defined as the ratio of the number of $\tau$ candidates passing the nominal identification criteria to the number of $\tau$ candidates failing those identification criteria, in a given control region. Fake factors are usually binned in the $p_{T}, \eta$ and/or number of associated tracks of the $\tau$ candidates. Since the fake- $\tau$ background arises from both multijet and $t t$ events, with different quark-gluon compositions, fake factors must be determined separately in control regions enriched in these two processes and later weighted according to the fraction of multijet events in the region enriched in misidentified $\tau$ candidates where they are applied. In the CMS search, the final discriminant is the transverse mass of either the hadronic $\tau$ object in the $\tau+$ jets channel or the charged lepton $\ell=e, \mu$ otherwise:

$$
m_{T}(\tau, \ell)=\sqrt{2 p_{T}^{\tau, \ell} E_{T}^{\text {miss }} \cos \Delta \phi_{\text {miss }}},
$$

where $\Delta \phi_{\text {miss }}$ is the azimuthal angle between the transverse missing momentum and either the hadronic $\tau$ object or the charged lepton. The main backgrounds are further reduced by using additional selections on azimuthal angle differences and, in the $\tau+$ jets channel, an event categorisation based on a $\tau$ polarisation variable. Event categorisations according to the jet and $b$-jet multiplicities are employed in the leptonic channels in order to further enhance the signal sensitivity and to constrain the backgrounds. In total, $36 m_{T}$ distributions are used in the statistical analysis of the search for $H^{ \pm} \rightarrow \tau v$ in CMS. One of the two distributions in the $\tau+$ jets channel is shown in Figure 19a.

In the ATLAS search, kinematic variables that distinguish between the signal and backgrounds are combined into BDT discriminants, and their output scores are then used in order to separate the $H^{ \pm} \rightarrow \tau v$ signal events from the SM background processes. The BDTs are trained in five different $H^{ \pm}$mass ranges, separately for the $\tau+$ jets and $\tau+$ lepton events. For $m_{H^{ \pm}}$in the range $90-400 \mathrm{GeV}$, the BDT training also depends on whether the leading $\tau$ candidate has one or three associated tracks, because the asymmetry of energies carried by the charged and neutral pions in the single-track $\tau$-lepton decay can be used as a proxy for its polarisation and is thus one of the BDT input variables. A BDT output score distribution in the $\tau+$ lepton channel is shown in Figure 19b. In order to constrain the $t t$ background, the ATLAS search also includes a single-bin control region, which is defined with the same event selection as the $\tau+$ lepton channel, but with the requirement of an $e \mu$ pair in place of the $e / \mu+\tau$ pair.

As illustrated in Figure 20, the sensitivity of the two searches for $H^{ \pm} \rightarrow \tau v$ is similar in terms of expected upper limits on the product of the cross-section and the branching fraction at $95 \%$ CL, and no significant excess of data with respect to the SM backgrounds is observed in neither ATLAS nor CMS searches. The observed upper limits reported by ATLAS (CMS) as a function of the $H^{ \pm}$mass range from $4.2 \mathrm{pb}$ at $90 \mathrm{GeV}$ to $2.5 \mathrm{fb}$ at $2 \mathrm{TeV}$ (from $6 \mathrm{pb}$ at $80 \mathrm{GeV}$ to $5 \mathrm{fb}$ at $3 \mathrm{TeV}$ ). In the low $H^{ \pm}$mass regime, these translate into limits on the branching fraction product $B\left(t \rightarrow b H^{ \pm}\right) \times B\left(H^{ \pm} \rightarrow \tau v\right.$ ) from $0.25 \%$ (at $90 \mathrm{GeV}$ ) to $0.03 \%$ (at $160 \mathrm{GeV}$ ) for ATLAS, and from $0.36 \%$ (at $80 \mathrm{GeV}$ ) to $0.08 \%$ (at $160 \mathrm{GeV}$ ) for CMS. These results were interpreted in various benchmark scenarios of the MSSM. Figure 21 shows that, in the hMSSM, the bulk of the sensitivity at low $m_{H^{ \pm}}$and high $\tan \beta$ is driven by the $H^{ \pm} \rightarrow \tau v$ search channel, while the $H^{ \pm} \rightarrow t b$ channel, discussed in the next sub-section, provides additional sensitivity to charged Higgs bosons for $m_{H^{ \pm}}$above the top-quark mass at low $\tan \beta$. 


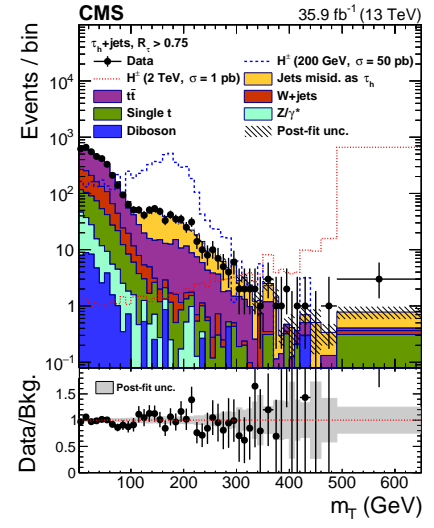

(a)

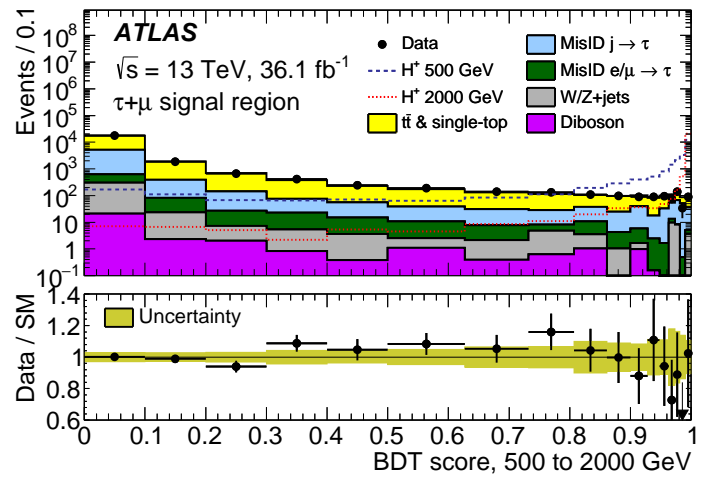

(b)

Figure 19. (a) Distribution of $m_{T}$ in the $\tau+$ jets channel of the CMS search for $H^{ \pm} \rightarrow \tau v$ after a background-only fit to the data in the category with $R_{\tau}=p_{T}^{\text {track }} / p_{T}^{\tau}>0.75$ [135], (b) distribution of the output score of the BDT trained using signal samples with $m_{H^{ \pm}}$in the range $500-2000 \mathrm{GeV}$, as obtained in the signal region of the $\tau+$ muon sub-channel in the ATLAS search for $H^{ \pm} \rightarrow \tau v$ after a background-only fit to the data [134]. Illustrative $H^{ \pm}$signals are shown in both cases. Copyright 2021 CERN for the benefit of the CMS Collaboration (a) and ATLAS Collaboration (b). CC-BY-4.0 license.

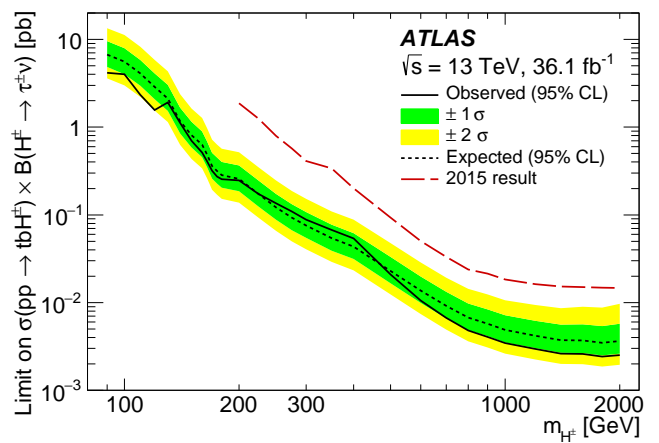

(a)

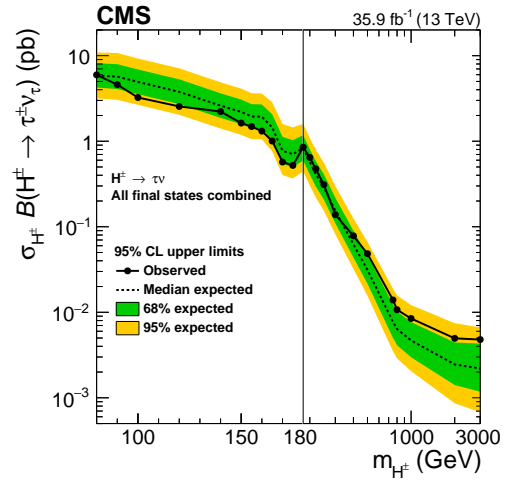

(b)

Figure 20. Expected and observed 95\% CL limits on $\sigma\left(p p \rightarrow t b H^{ \pm}\right) \times B\left(H^{ \pm} \rightarrow \tau v\right)$ as a function of the charged Higgs boson mass, as reported by ATLAS and CMS in, respectively, [134,135]. Copyright 2021 CERN for the benefit of the ATLAS Collaboration (a) and CMS Collaboration (b). CC-BY-4.0 license.

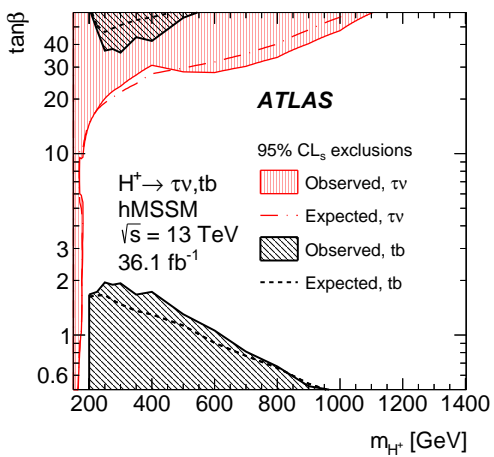

Figure 21. Expected and observed $95 \%$ CL limits on $\tan \beta$ as a function of $m_{H^{ \pm}}$in the hMSSM. The limits are reported as a superposition of the search results obtained by ATLAS for $H^{ \pm} \rightarrow \tau v$ [134] and $H^{ \pm} \rightarrow t b$ [136]. The exclusion limits are shown only for $m_{H^{ \pm}}$above $150 \mathrm{GeV}$ and $\tan \beta$ in the range of $0.5-60$, where theoretical predictions are available. Copyright $2021 \mathrm{CERN}$ for the benefit of the ATLAS Collaboration. CC-BY-4.0 license. 


\subsection{2. $H^{ \pm} \rightarrow t b$}

The $H^{ \pm} \rightarrow t b$ decay mode is instrumental to the search for charged Higgs bosons above the top-quark mass, as it has the largest branching fraction in many extended Higgs sector scenarios. Both the ATLAS and CMS Collaborations have searched for the production of $H^{ \pm} \rightarrow t b$ in association with top- and bottom-quarks (Figure 18b). This yields experimental signatures with a large multiplicity of hadronic jets, among which at least three can be identified as originating from a $b$-hadron. The expected $b$-jet multiplicity depends on whether $b$-quarks are explicitly included in the parton distribution functions or not. The final state also contains two $W$ bosons, one from the charged Higgs boson chain $\left(H^{ \pm} \rightarrow t b \rightarrow(\mathrm{Wb}) b\right)$ and the other from the direct decay of the associated top-quark. Given the complex multijet signatures with zero, one or two charged leptons, the dominant and most challenging background in searches for $H^{ \pm} \rightarrow t b$ is the production of $t t$ pairs with additional jets from initial- or final-state radiation, as well as additional gluon splitting that can enhance the $b$-jet multiplicity. In all ATLAS and CMS searches, this background is split into three components. The $t t+b(b)$ and $t t+c(c)$ labels refer to generated events that have, respectively, at least one $b$-jet or at least one $c$-jet and no $b$-jet within the acceptance. Otherwise, events are enriched in light-flavour jets and labelled as $t t+L F$. While minor in the leptonic channels, the contribution of multijet processes to the total background is significant in the fully hadronic channel.

In ATLAS, two searches for $H^{ \pm} \rightarrow t b$ based on the Run- 2 dataset at $13 \mathrm{TeV}$ have been conducted: the first one uses $36 \mathrm{fb}^{-1}$ of collision data and considers multijet signatures with one or two electrons or muons [136], followed by an updated analysis of the full Run-2 dataset $\left(139 \mathrm{fb}^{-1}\right)$ using multijet signatures with exactly one electron or muon [137]. In CMS, two searches for $H^{ \pm} \rightarrow t b$ have been performed, both based on $36 \mathrm{fb}^{-1}$ of collision data: one considers multijet signatures with one or two electrons or muons [138], whereas the other targets fully hadronic multijet signatures [139].

If at least one of the final-state $W$ bosons decays to an electron or muon (directly or via a $\tau$-lepton), the ATLAS and CMS searches make use of single-lepton triggers to record the events. Then, events are categorised according to their jet and $b$-jet multiplicities in order to eventually improve the sensitivity of the statistical analysis by constraining various background components and some of the systematic uncertainties. In the ATLAS search of [137], four signal regions are considered: $5 j 3 b, 5 j \geq 4 b, \geq 6 j 3 b$ and $\geq 6 j \geq 4 b$, where $\mathrm{Xj} Y \mathrm{~b}$ means that the event contains $\mathrm{X}$ jets, of which $\mathrm{Y}$ are $b$-tagged with an efficiency of $70 \%$ in simulated $t t$ events. The CMS search uses nine signal regions for single-lepton events (4j1b, 4j2b, 4j $\geq 3 b, 5 j 1 b, 5 j 2 b, 5 j \geq 3 b, \geq 6 j 1 b, \geq 6 j 2 b$ and $\geq 6 j \geq 3 b$ ) and eight in dilepton events ( $2 j 1 b, 2 j 2 b, 3 j 1 b, 3 j 2 b, 3 j 3 b, \geq 4 j 1 b, \geq 4 j 2 b$ and $\geq 4 j \geq 3 b$ ). Both ATLAS and CMS also use additional control regions in order to derive data-driven corrections for the backgrounds. In each category, a set of kinematic variables is selected to enhance the separation between the $H^{ \pm} \rightarrow t b$ signal and the SM background processes, and they are subsequently included in a multi-variate classifier. The ATLAS search of [137] uses a neural network (NN) algorithm, and its training is performed separately in each analysis region, including all $H^{ \pm}$signal samples with the value of $m_{H^{ \pm}}$as a parameter. The CMS search uses separate classifiers for the single-lepton and dilepton final states. In the former case, a BDT classifier trained separately for each $m_{H^{ \pm}}$hypothesis in an inclusive $\geq 5 \mathrm{j} \geq 2 \mathrm{~b}$ single-lepton signal region is used. On the other hand, when searching for $H^{ \pm} \rightarrow t b$ in the dilepton final states, CMS exploits a deep neural network (DNN) classifier trained in an inclusive $\geq 3 \mathbf{j} \geq 1 \mathrm{~b}$ region and parameterised as a function of $m_{H^{ \pm}}$, as well as of the jet and $b$-jet multiplicities.

Figure 22a,b show the NN output distributions after a signal+background fit with $m_{H^{ \pm}}=800 \mathrm{GeV}$ in two single-lepton signal regions ( $5 \mathrm{j} 3 \mathrm{~b}$ and $\geq 6 \mathrm{j} \geq 4 \mathrm{~b}$ ) of the ATLAS search for $H^{ \pm} \rightarrow t b$, thereby illustrating differences in background composition between different jet and $b$-jet multiplicity categories. Figure $22 \mathrm{c}$ shows the event yields in the various dilepton signal regions of the CMS search after a background-only fit to the data and 
Figure $22 \mathrm{~d}$ shows an illustrative DNN output distribution in the corresponding $\geq 3 \mathrm{j} \geq 3 \mathrm{~b}$ signal region.

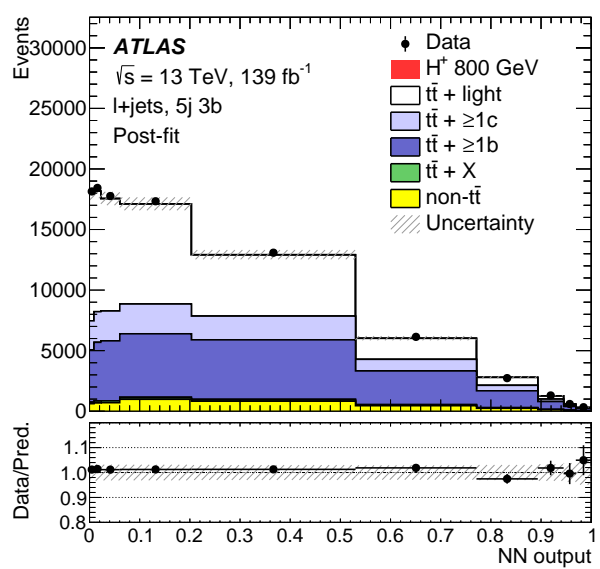

(a)

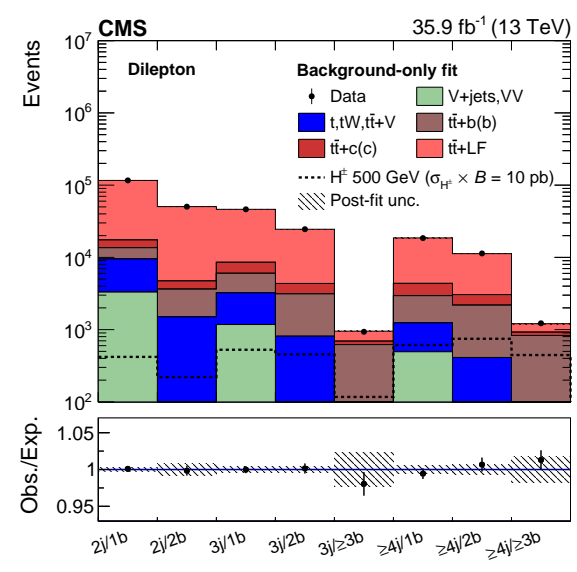

(c)

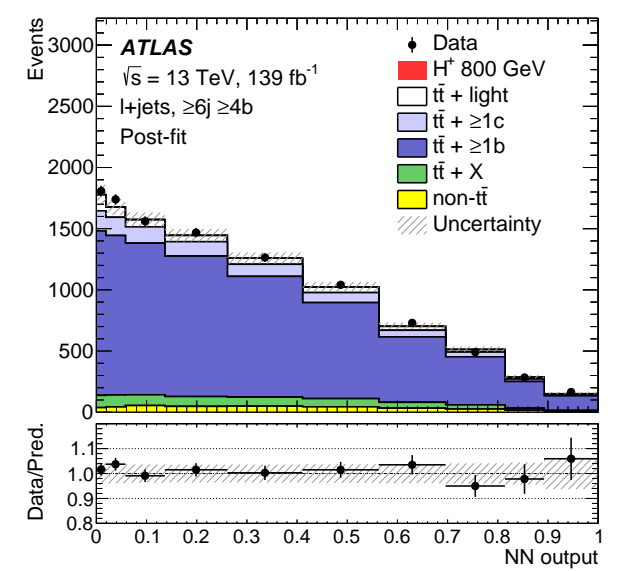

(b)

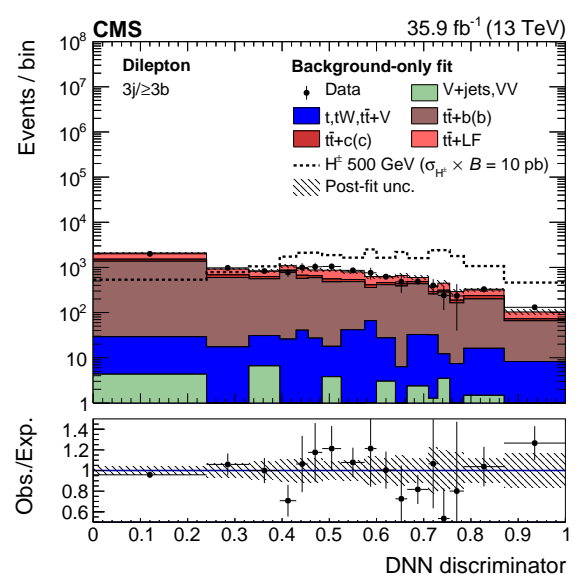

(d)

Figure 22. Distributions of the NN output in the (a) $5 j 3 b$ and (b) $\geq 6 j \geq 4 b$ single-lepton categories of the ATLAS $H^{ \pm} \rightarrow t b$ search [137] after a signal+background fit with $m_{H^{ \pm}}=800 \mathrm{GeV}$, and distributions of (c) the event yields across dilepton regions and (d) the DNN output in the $\geq 3 \mathrm{j} \geq 3 \mathrm{~b}$ signal category of the CMS $H^{ \pm} \rightarrow t b$ search [138]. Copyright 2021 CERN for the benefit of the ATLAS Collaboration $(\mathbf{a}, \mathbf{b})$ and CMS Collaboration (c,d). CC-BY-4.0 license.

In another search for $H^{ \pm} \rightarrow t b$, CMS targeted fully hadronic final states arising from a signal that contains $W \rightarrow q q^{\prime}$ in the decay chains of both the charged Higgs boson and the associated top-quark. Two distinct topologies were considered, where the $H^{ \pm}$decay products are either resolved or, for $m_{H^{ \pm}}>5 m_{t}$, merged. In that search, data were collected by applying trigger requirements based on multiple small-radius jets and/or the scalar sum $H_{T}$ of their transverse momenta, as well as by using a large-radius jet trigger with requirements on both the $p_{T}$ and mass of that jet. The analysis of the resolved topologies relies on the identification of two top-quark candidates in events with at least seven smallradius jets, of which at least three are $b$-tagged, by using a multi-variate boosted decision tree with gradient boost (BDTG) classifier. Subsequently, the invariant mass of the $H^{ \pm}$ candidate is reconstructed from the resolved top-quark candidate with the highest $p_{T}$ and the $b$-tagged jet with the highest $p_{T}$ that is not used in the reconstruction of the two selected top-quark candidates. The analysis of the merged topologies uses large-radius jets to reconstruct and identify the decay products of boosted $W$ bosons and top-quarks, together with information on the jet mass and substructure, as well as on the $b$-tagging of the constituent sub-jets. Subsequently, events are categorised according to whether a 
top-quark is reconstructed from a single large-radius jet or from a large-radius $W$ boson candidate and a $b$-tagged jet, according to the $b$-tagging of the constituent sub-jets and according to the multiplicity of small-radius jets and $b$-tags. The main backgrounds for this search are multijet processes and $t t$ production with additional jets. They are estimated with either simulation or data-driven techniques, and their normalisations and shapes are constrained through the use of dedicated background-enriched control regions in the final statistical analysis.

In the absence of statistically significant deviations from the SM predictions, both ATLAS and CMS have set limits on the production cross-section of $p p \rightarrow t b H^{ \pm}$times the branching fraction of the $H^{ \pm} \rightarrow t b$ decay mode. The CMS upper limits at 95\% CL are shown in Figure 23a: they correspond to the statistical combination of the searches performed in the leptonic and fully hadronic channels with $36 \mathrm{fb}^{-1}$ of data at $13 \mathrm{TeV}$. The single-lepton final state has the best sensitivity across the whole charged Higgs boson mass range $(0.2-3 \mathrm{TeV})$. The dilepton channel contributes at low $m_{H^{ \pm}}$values, typically below $1.5 \mathrm{TeV}$, while the fully hadronic channel improves the overall sensitivity of the search at large $m_{H^{ \pm}}$values. The limits set by ATLAS both on the cross-section of $p p \rightarrow t b H^{ \pm}$ times the branching fraction of $H^{ \pm} \rightarrow t b$ and in the hMSSM parameter space are shown in Figure $23 b, c$, respectively. Compared to the previous results of the same search channel, reported in Figure 21, a broader region is excluded.

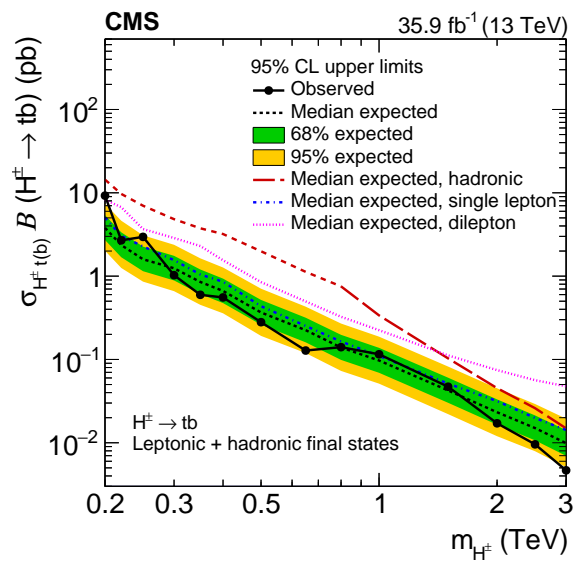

(a)

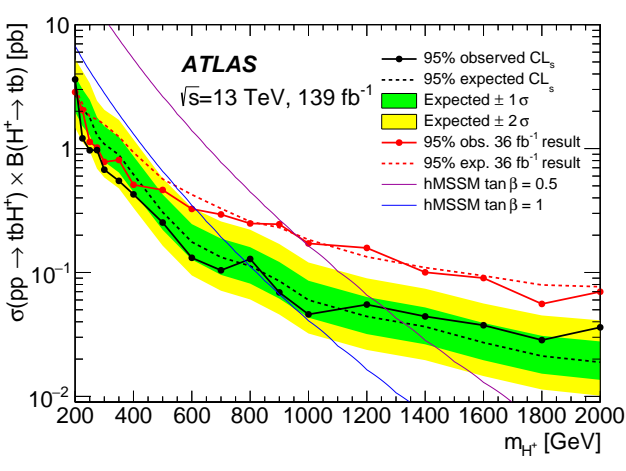

(b)

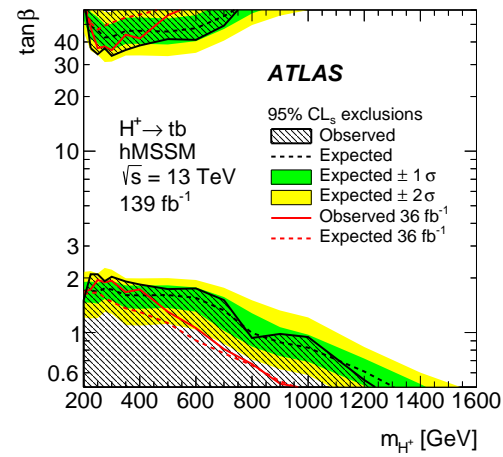

(c)

Figure 23. Observed and expected 95\% CL limits for the production of $H^{ \pm} \rightarrow t b$ in association with a top-quark and a bottom-quark (a) through a statistical combination of the CMS leptonic and fully hadronic search channels with $36 \mathrm{fb}^{-1}$ of data at $13 \mathrm{TeV}$ [139], (b,c) in the ATLAS single-lepton search channel based on $139 \mathrm{fb}^{-1}$ of data at $13 \mathrm{TeV}$ [137]. Copyright $2021 \mathrm{CERN}$ for the benefit of the CMS Collaboration (a) and ATLAS Collaboration (b,c). CC-BY-4.0 license. 


\subsection{3. $H^{ \pm} \rightarrow c s / c b$}

Besides the search for $H^{ \pm} \rightarrow \tau v$ reported in Section 4.1.1, two other decay modes of the charged Higgs boson have been investigated in order to probe the $m_{H^{ \pm}}$range below the top-quark mass. ATLAS has searched for $H^{ \pm} \rightarrow c b$ in $139 \mathrm{fb}^{-1}$ of $13 \mathrm{TeV}$ data [140], while a search for $H^{ \pm} \rightarrow c s$ has been conducted by CMS based on a partial Run-2 dataset of $36 \mathrm{fb}^{-1}$ in [141]. Both searches use multijet final states with an electron or muon from the associated leptonic top-quark decay, which is used for triggering purposes.

In the ATLAS search for $H^{ \pm} \rightarrow c b$, given the large jet and $b$-tagged jet multiplicities, an event categorisation is employed, similarly to the $H^{ \pm} \rightarrow t b$ single-lepton search channel. Six event categories are used in the statistical analysis: $4 j 3 b, 5 j 3 b, 6 j 3 b, 4 j 4 b, 5 j \geq 4 b$ and $6 \mathrm{j} \geq 4 \mathrm{~b}$, while three additional regions with a looser $b$-tagging requirement are used to correct the modelling of the $t t$ background. Neural networks parameterised as a function of $m_{H^{ \pm}}$(by incorporating the $H^{ \pm}$mass information as a label during training) are used to separate the signal from the SM background. As it is dominated by the contribution of $t t$ with additional jets, this background is again split into three components, $t t+b(b)$, $t t+c(c)$ and $t t+\mathrm{LF}$, which can be constrained separately in the different event categories. In particular, the regions with three $b$-tagged jets constrain the leading shape uncertainties of the $t t+\mathrm{LF}$ and $t t+b(b)$ backgrounds, while the regions with four $b$-tagged jets help adjust the normalisation of $t t+b(b)$. In the absence of a statistically significant excess of data with respect to the SM expectation, exclusion limits at 95\% CL are set on the product of the branching fractions of $t \rightarrow b H^{ \pm}$and $H^{ \pm} \rightarrow c b$ between $0.15 \%$ and $0.42 \%$ across the $m_{H^{ \pm}}$range of $60-160 \mathrm{GeV}$, as shown in Figure $24 \mathrm{a}$.

In the CMS search for $H^{ \pm} \rightarrow c s$, the topology of interest consists of a leptonically decaying $W$ boson and four jets (including two $b$-tagged and one $c$-tagged jets). In order to identify semileptonic $t$ decays, a kinematic fit is employed, the output of which is exactly four jets (two $b$-tagged, one from each of the leptonic and hadronic top-quark decays, and two non-b-tagged jets from the hadronic top-quark decay), one lepton and a neutrino. The invariant mass of the two non- $b$-tagged jets is subsequently used as the final discriminant. The events are further split into three exclusive categories based on $c$-tagging requirements for the two non- $b$-tagged jets. In the absence of a statistically significant excess of data with respect to the SM expectation, exclusion limits at 95\% CL are set on the branching fraction of the decay $t \rightarrow b H^{ \pm}$under the assumption that $B\left(H^{ \pm} \rightarrow c s\right)=1$ : they vary between $0.25 \%$ and $1.68 \%$ across the $m_{H^{ \pm}}$range of $80-160 \mathrm{GeV}$, as shown in Figure $24 \mathrm{~b}$.

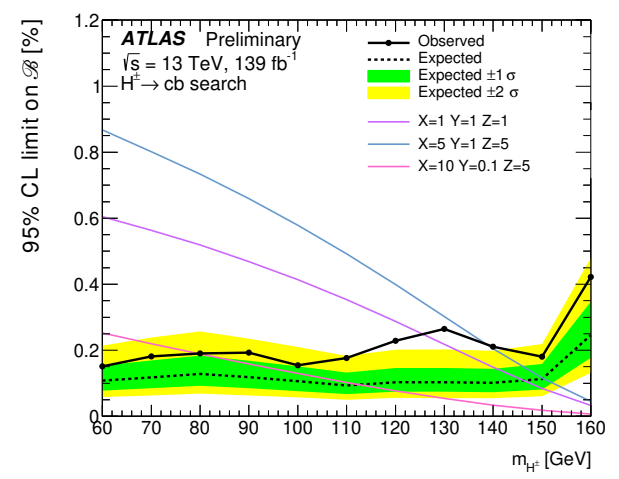

(a)

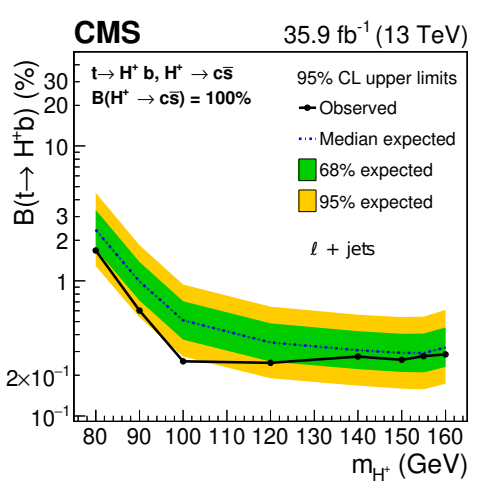

(b)

Figure 24. Observed and expected 95\% CL limits for the production of $t \rightarrow b H^{ \pm}$in (a) the $H^{ \pm} \rightarrow c b$ decay mode, as reported by ATLAS in [140], and (b) the $H^{ \pm} \rightarrow c s$ decay mode, as reported by CMS in [141]. In the left-hand side plot, the superimposed lines correspond to three benchmarks of a threeHiggs-doublet model (3HDM), where the parameters X, Y and Z are functions of the Higgs doublet vacuum expectation values and the mixing angle between the charged Higgs bosons [142,143]. Copyright 2021 CERN for the benefit of the ATLAS Collaboration (a) and CMS Collaboration (b). CC-BY-4.0 license. 


\subsection{Search Results for Singly Charged Higgs Bosons in Bosonic Decay Channels}

4.2.1. $H^{ \pm} \rightarrow A W$

In the $m_{H^{ \pm}}$range below the top-quark mass, fermionic decays of charged Higgs bosons are not always dominant. In particular, any generic 2HDM and, e.g., the NMSSM predict a large branching fraction for the decay of $H^{ \pm}$to a light CP-odd Higgs boson $A$ and a $W$ boson. Using $36 \mathrm{fb}^{-1}$ of $13 \mathrm{TeV}$ data, the CMS Collaboration has searched for $H^{ \pm} \rightarrow A W$ in decay cascades of $t t$ events with $е \mu \mu$ and $\mu \mu \mu$ final states [144]. Such signatures arise from a $t \rightarrow b H^{ \pm}$decay, where the charged Higgs boson subsequently decays to a $W$ boson and a light CP-odd Higgs boson $A \rightarrow \mu \mu$, while the additional electron or muon comes from the leptonic decay of one of the two $W$ bosons in the event. The ATLAS Collaboration recently performed a similar search in the e $\mu \mu$ final state, using $139 \mathrm{fb}^{-1}$ of Run-2 data [145].

Given the poor resolution of the $m_{H^{ \pm}}$reconstruction, a signal is searched for in the mass spectrum of two opposite-sign muons that are not compatible with a Z-boson decay. While there is no ambiguity in assigning such muon pairs to the $A$ candidate in $e \mu \mu$ events, additional selection criteria are employed by CMS to assign the muons to the $A$ and $W$ bosons in the case of $\mu \mu \mu$ final states. In the CMS search, backgrounds with at least one nonprompt lepton arising from a jet are estimated by applying data-driven extrapolation factors on events in which the leptons fail tight identification criteria but pass loose identification criteria. In the ATLAS search, all backgrounds are simulated, however the overall yields of $t t, t t Z$ and $Z+$ heavy-flavour jets are constrained with data by using a profile likelihood fit in three background-enriched control regions. Both ATLAS and CMS then test the presence of $H^{ \pm} \rightarrow A W$ events in the dimuon mass distribution of the $A$ candidate by comparing the measured and predicted event yields within a $m_{\mu \mu}$ window that is specific to each mass hypothesis. Figure 25 shows the dimuon mass distribution predicted and measured by CMS, as well as example upper limits on the product of branching fractions for $t \rightarrow b H^{ \pm}, H^{ \pm} \rightarrow A W$ and $A \rightarrow \mu \mu$. As a comparison, the observed (expected) upper limits obtained by ATLAS for the $m_{H^{ \pm}}=160 \mathrm{GeV}$ mass hypothesis range from $1.0(1.6) \times 10^{-6}$ to $3.6(3.2) \times 10^{-6}$ for $m_{A}$ varying between $15 \mathrm{GeV}$ and $75 \mathrm{GeV}$, however with nearly four times more data. The ATLAS results are further interpreted in a type-I 2HDM as lower limits on $\tan \beta$ in the $\left(m_{A}, m_{H^{ \pm}}\right)$plane, as shown in Figure 26.

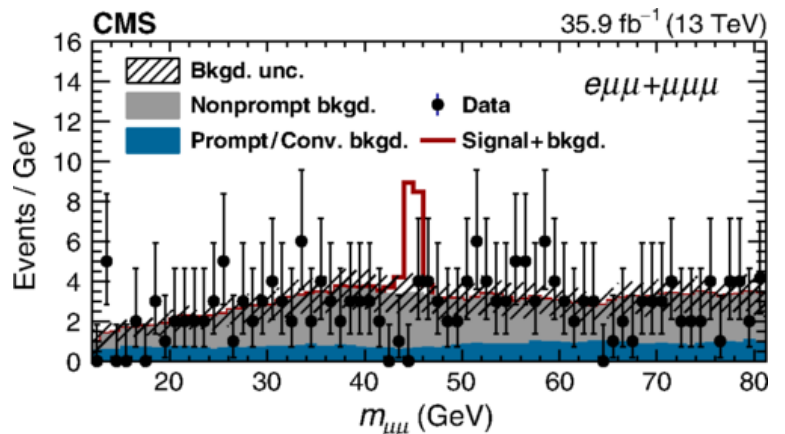

(a)

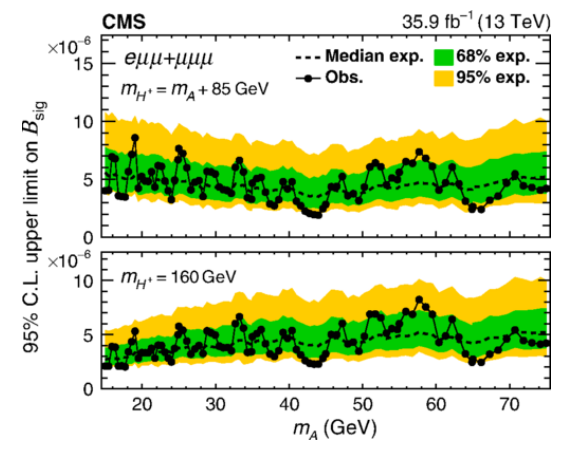

(b)

Figure 25. (a) Mass spectrum of the dimuon system associated to the $A$ candidate and (b) $95 \%$ CL limits on the product of branching fractions for $t \rightarrow b H^{ \pm}, H^{ \pm} \rightarrow A W$ and $A \rightarrow \mu \mu$ for two $m_{H^{ \pm}}$hypotheses, as reported by CMS in [144]. Copyright 2021 CERN for the benefit of the CMS Collaboration. CC-BY-4.0 license. 


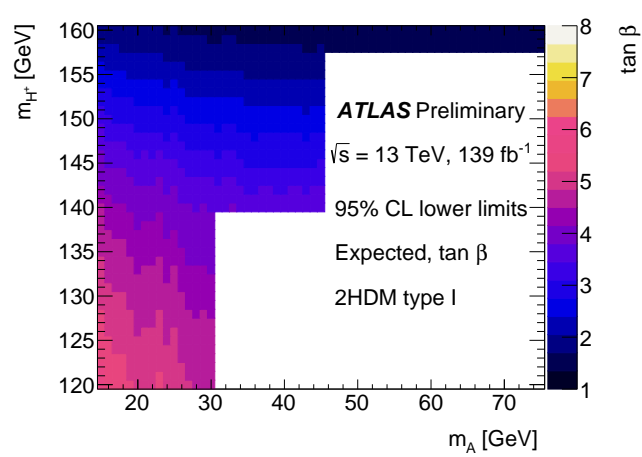

(a)

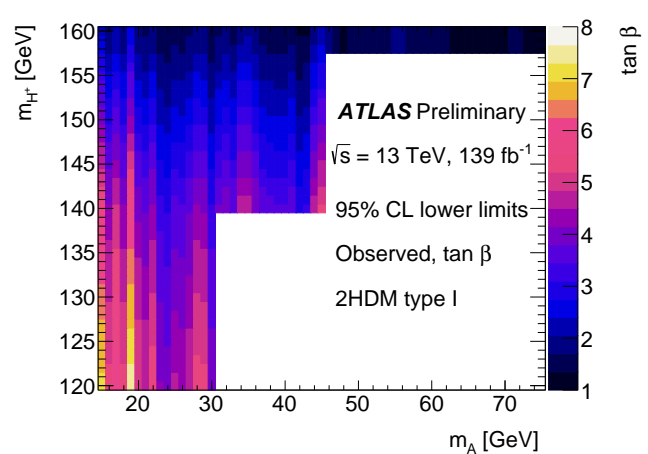

(b)

Figure 26. Interpretation of the ATLAS search results of [145] as (a) expected and (b) observed lower limits on $\tan \beta$ in a type-I 2HDM. The empty region corresponds to an off-shell $W$ boson. Copyright 2021 CERN for the benefit of the ATLAS Collaboration. CC-BY-4.0 license.

\subsubsection{VBF Production of $H^{ \pm} \rightarrow W Z$}

While the decay $H^{ \pm} \rightarrow W Z$ is not allowed in the 2HDM, extended Higgs sectors containing additional weak isospin triplets do give rise to couplings of charged Higgs bosons to $W$ and $Z$ bosons at tree-level. For instance, in parts of the parameter space of the Georgi-Machacek model, several of the additional Higgs bosons couple only to vector bosons, hence the VBF production of a charged Higgs boson followed by $H^{ \pm} \rightarrow W Z$ becomes dominant (see Figure 27a). This search channel was explored by ATLAS in [146] using $36 \mathrm{fb}^{-1}$ of $13 \mathrm{TeV}$ data and, more recently, by CMS using $137 \mathrm{fb}^{-1}$ of Run-2 data [147]. By considering leptonic decays for both $W$ and $Z$ bosons, signatures consisting of three leptons (electron and/or muons) in association with two forward jets are employed to search for the bosonic decay $H^{ \pm} \rightarrow W Z$. In the CMS search, the event selection requires a pair of central oppositely charged same-flavour leptons compatible with a Z-boson decay and a third lepton, with a total invariant mass $m_{\ell \ell \ell}>100 \mathrm{GeV}$, missing transverse energy in excess of $30 \mathrm{GeV}$ and a VBF topology characterised by the two highest- $p_{T}$ jets having a mass $m_{j j}>500 \mathrm{GeV}$ and a separation in pseudorapidity $\left|\Delta \eta_{j j}>2.5\right|$. The main backgrounds arise from the production of a diboson system in association with two jets, which can proceed via either electroweak interaction or QCD radiation. In addition, the associated production of a $Z$ boson and a top-quark $(t Z q)$ is also an important background. These processes are estimated through a combination of methods based on simulation and on background-enriched control regions in data, which are obtained by inverting some of the event selection criteria. A binned maximum-likelihood fit of signal and control regions is used for signal extraction. It is based on two-dimensional distributions in the mass plane $\left(m_{T}^{V V}, m_{j j}\right)$, where $m_{T}^{V V}$ is the diboson transverse mass reconstructed from the four-momenta of the charged leptons and the missing transverse momentum. In the absence of any statistically significant excess of data above the background prediction, exclusion limits at $95 \% \mathrm{CL}$ are set on the product of the charged Higgs boson cross-section and the branching fraction $\sigma_{\mathrm{VBF}}\left(H^{ \pm}\right) \times B\left(H^{ \pm} \rightarrow W Z\right)$ as a function of $m_{H^{ \pm}}$, as shown in Figure 27b. 


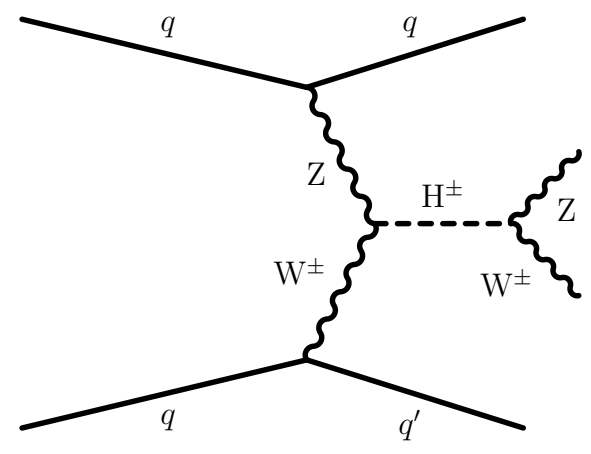

(a)

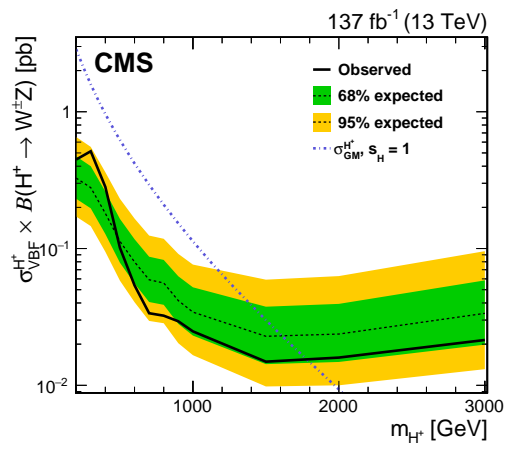

(b)

Figure 27. (a) Feynman diagram for the VBF production of a charged Higgs boson decaying via $H^{ \pm} \rightarrow$ WZ. (b) Expected and observed limits on $\sigma_{\mathrm{VBF}}\left(H^{ \pm}\right) \times B\left(H^{ \pm} \rightarrow \mathrm{WZ}\right)$ at $95 \% \mathrm{CL}$ as a function of $m_{H^{ \pm}}$, as reported in [147]. Copyright 2021 CERN for the benefit of the CMS Collaboration. CC-BY-4.0 license.

\subsection{Search Results for Doubly Charged Higgs Bosons}

Doubly charged Higgs bosons appear in extended Higgs sectors that contain weak isospin triplets, such as the Georgi-Machacek model described in Section 2.3. In order to probe this model, the CMS Collaboration has searched for the VBF production of a doubly charged Higgs boson followed by a $H^{ \pm \pm} \rightarrow W^{ \pm} W^{ \pm}$decay, using $137 \mathrm{fb}^{-1}$ of $13 \mathrm{TeV}$ data, as reported in the same paper as the search for VBF production of $H^{ \pm} \rightarrow W Z$ [147]. The analysis differs from that reported above by targeting one central same-sign lepton pair instead of three leptons, whereas the requirements on the missing transverse momentum and the forward jets remain the same. The $H^{ \pm \pm} \rightarrow W^{ \pm} W^{ \pm}$signal extraction uses the same maximum-likelihood fit, also based on two-dimensional distributions in the mass plane $\left(m_{T}^{V V}, m_{j j}\right)$, but it is based on complementary signal regions. This is illustrated in Figure 28a, where the bins 1-32 show events in the $W^{ \pm} W^{ \pm}$signal region (four bins in $m_{j j}$ times eight in $m_{T}^{V V}$ ), the bins 33-46 show events in the $W Z$ signal region (two bins in $m_{j j}$ times seven in $\left.m_{T}^{V V}\right)$, and the other twelve bins show events in three background-enriched control regions. In the absence of any statistically significant excess of data above the background prediction, $95 \%$ CL exclusion limits are set on the product $\sigma_{\mathrm{VBF}}\left(H^{ \pm \pm}\right) \times B\left(H^{ \pm \pm} \rightarrow W^{ \pm} W^{ \pm}\right)$. Such results, together with those reported in the previous sub-section, can be interpreted in the context of the Georgi-Machacek model by including the simultaneous contributions of the singly and doubly charged Higgs bosons, as shown in Figure 28b.

Recently, ATLAS searched for doubly charged Higgs bosons by using multi-lepton final states that arise from either the production of $H^{ \pm \pm}$pairs or the associated production of one $H^{ \pm \pm}$with a singly charged Higgs boson, with the subsequent decays $H^{ \pm \pm} \rightarrow W^{ \pm} W^{ \pm}$ and $H^{ \pm} \rightarrow W Z$, as shown in Figure 29a,b [148]. The Higgs sector extension probed by ATLAS adds only one triplet of scalar fields [149] while allowing to generate neutrino masses through a type-II see-saw mechanism [150]. In that scenario, the production and decay modes considered by ATLAS are dominant for vacuum expectation values of the triplet typically above $1 \mathrm{MeV}$ [151]. It should be noted that the ATLAS search of [148] is sensitive to parts of the parameter space of the Georgi-Machacek model, despite this interpretation not being included in the reference. The four final-state vector bosons are identified by the presence of multiple charged leptons (electrons or muons), missing transverse momentum and jets. The ATLAS analysis uses the full Run- 2 dataset and three mutually exclusive search regions are defined, where the absolute value of the sum of lepton charges is two $\left(2 \ell^{\mathrm{sc}}\right)$, one $(3 \ell)$ and two or zero $(4 \ell)$. Further event selections are then imposed in each channel to optimise the sensitivity to the hypothesised signals, by, e.g., taking advantage of differences in the background composition and in the lepton-flavour composition between signal and backgrounds. While background processes with a prompt lepton coming from a 
$W$ or $Z$ boson are determined with simulation, processes with nonprompt leptons and electron charge-flip are estimated with data-driven methods. Figure 29c shows a comparison of the predicted and measured event yields after a background-only fit in the three search regions and for various signal mass hypotheses.

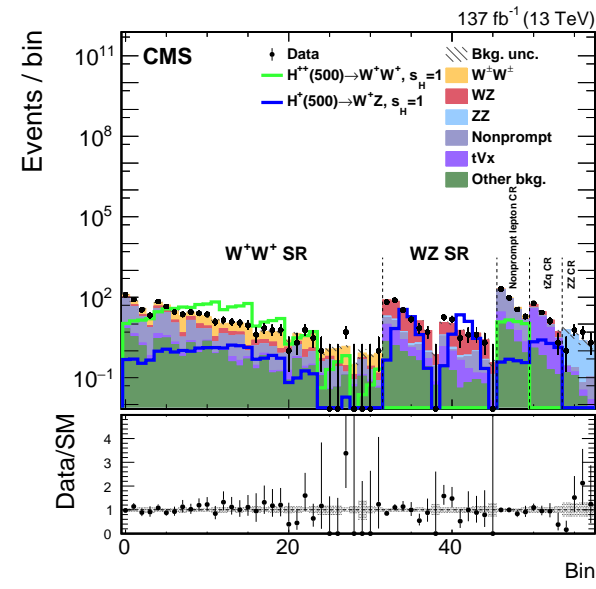

(a)

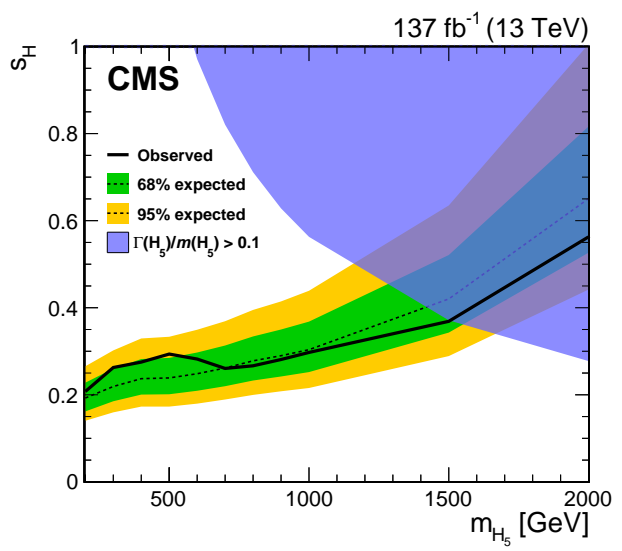

(b)

Figure 28. (a) Event yields in the bins entering the maximum-likelihood fit used in the CMS search for VBF production of $H^{ \pm \pm} \rightarrow W^{ \pm} W^{ \pm}$; see the text for details. (b) Expected and observed 95\% CL limits on $s_{H}$ as a function of the fiveplet mass parameter $m_{H 5}$ in the Georgi-Machacek model [147]. Copyright 2021 CERN for the benefit of the CMS Collaboration. CC-BY-4.0 license.

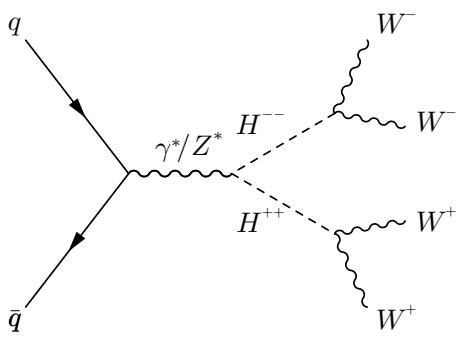

(a)

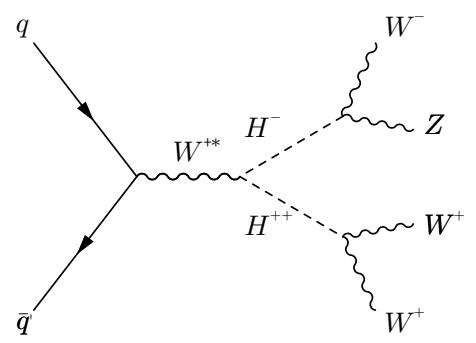

(b)

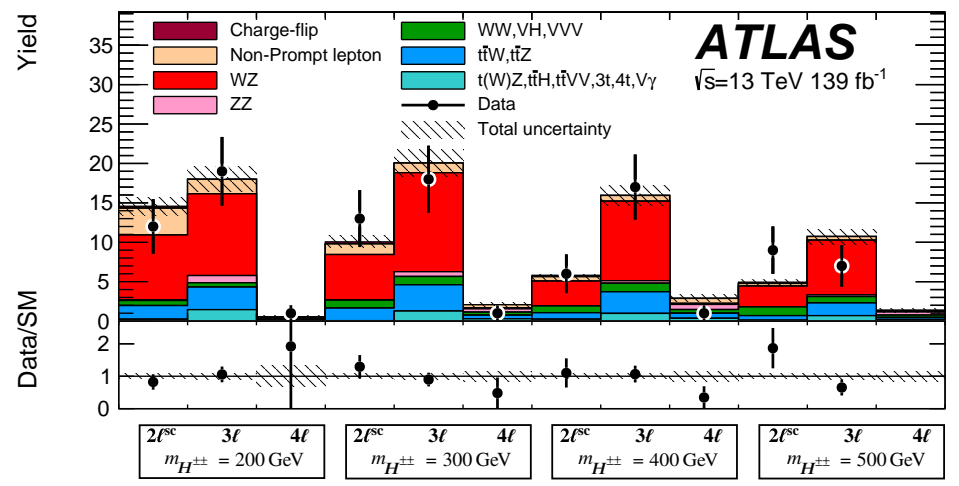

(c)

Figure 29. Feynman diagrams for (a) pair production and (b) $H^{ \pm}$-associated production of a doubly charged Higgs boson. (c) Expected and observed event yields in the $2 \ell^{\mathrm{sc}}, 3 \ell, 4 \ell$ signal regions considered in the ATLAS search of [148]. Copyright 2021 CERN for the benefit of the ATLAS Collaboration. CC-BY-4.0 license. 
In the absence of any significant excess of data above the background prediction, exclusion limits are set on the cross-sections for $H^{ \pm \pm} H^{ \pm \pm}$and $H^{ \pm \pm} H^{\mp}$ production. These are shown in Figure 30, after statistical combination of the $2 \ell^{\mathrm{sc}}, 3 \ell, 4 \ell$ signal regions. The corresponding lower limit on the mass of the doubly charged Higgs boson at 95\% CL is $350 \mathrm{GeV}$ for the pair production mode and $230 \mathrm{GeV}$ for the associated production mode.

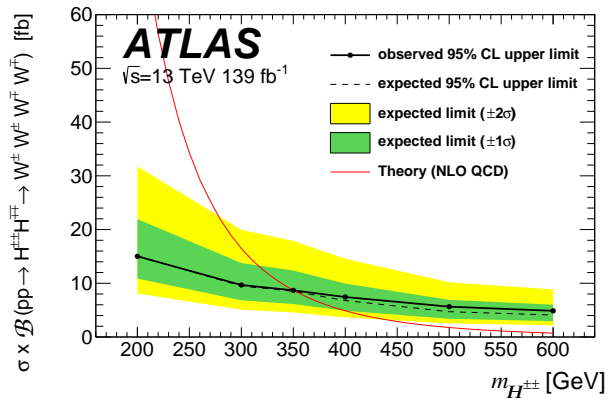

(a)

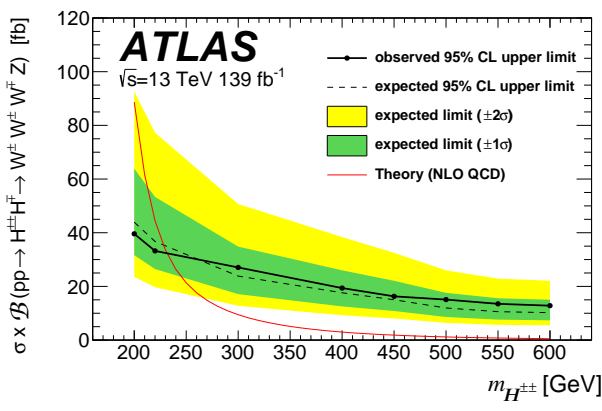

(b)

Figure 30. Expected and observed 95\% CL limits on the cross-section for (a) $H^{ \pm \pm} H^{ \pm \pm}$and (b) $H^{ \pm \pm} H^{\mp}$ production, with subsequent bosonic decays of the charged Higgs bosons, as reported by ATLAS in [148]. Copyright 2021 CERN for the benefit of the ATLAS Collaboration. CC-BY-4.0 license.

\section{Conclusions}

Searches for an extended Higgs sector at the LHC have evolved as data-taking has progressed during the past decade, from a couple of searches at the start of the LHC's operation to a large enterprise with many kinds of experimental signatures currently. In the years preceding the Higgs boson discovery in 2012, the main search channels were the MSSM-driven $A / H \rightarrow \tau \tau$ and $H^{ \pm} \rightarrow \tau v$. As more data were accumulated, the absence of any hints for supersymmetry and the increasingly accurate measurements of the properties of the discovered Higgs boson have motivated the search for a more diverse set of experimental signatures. More generic 2HDM-driven searches have been pursued, such as $A \rightarrow \mathrm{ZH}$ or $H^{ \pm} \rightarrow A W$. With larger datasets, an exploration of more complicated Higgs sectors, for instance with Higgs triplets, such as the Georgi-Machacek model, has been systematically pursued as well, through searches for doubly charged Higgs bosons and unique signatures as $H^{ \pm} \rightarrow Z W$. The ATLAS and CMS searches have not only focused on new heavy Higgs bosons, but also on lighter scalar states, e.g., through the search for exotic decays of the $125 \mathrm{GeV}$ Higgs boson, $h \rightarrow a a$.

The use of simple phenomenology benchmarks can be useful to compare the sensitivity of different signatures on the same footing. However, such a comparison may be misleading, since different Higgs sector structures lead to substantially different levels of sensitivity for any given experimental signature. ATLAS and CMS tend to use MSSM-inspired scenarios to make such sensitivity comparisons, as shown in Figure 31, which displays ATLAS and CMS summary plots for the hMSSM and $\mathrm{M}_{h}^{125}$ benchmark scenarios [114,152]. 


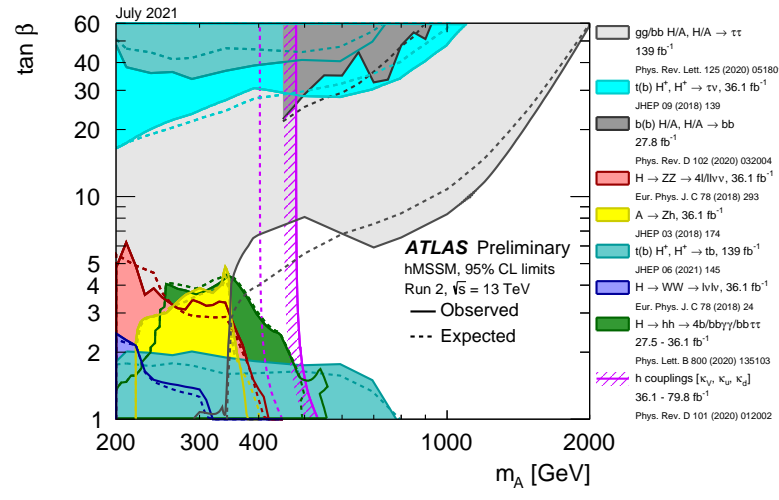

(a)

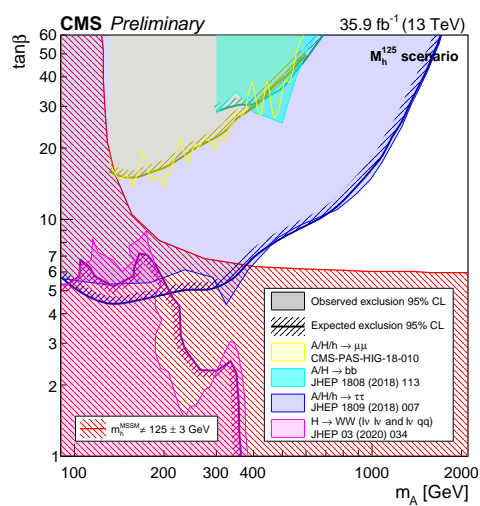

(b)

Figure 31. Examples of summary plots that compare several searches for extended Higgs sectors in the context of MSSM-inspired scenarios: (a) ATLAS summary plot for the hMSSM [114] and (b) CMS summary plot for the $\mathrm{M}_{h}^{125}$ scenario [152]. Copyright 2021 CERN for the benefit of the ATLAS Collaboration (a) and CMS Collaboration (b). CC-BY-4.0 license.

Even in these simple benchmarks, it can be seen that, despite the great progress achieved with respect to the pre-LHC era, when most of the parameter spaces shown in Figure 31 were not excluded, there are still regions to be explored, even in the sub-TeV Higgs boson mass range. In general, this unexplored parameter space becomes even larger in most of the other Higgs sector extensions. Therefore, the continuous improving of the numerous existing searches will definitely be a central part of the future LHC program. Several prospect studies related to searches for an extended Higgs sector at the HighLuminosity LHC, corresponding to an expected dataset of $14 \mathrm{TeV}$ proton-proton collisions with an integrated luminosity of $3000 \mathrm{fb}^{-1}$, can be found in [153].

Finally, the current absence of any evidence for new physics in direct searches further motivates the use of signature-focused searches. It may also motivate model builders to reconsider the interplay between flavour and Higgs physics, depending on the future results on the muon $g-2$ measurement [154] and current flavour anomalies reported in, e.g., $[155,156]$.

Author Contributions: Both authors contributed equally to the preparation of this review article. All authors have read and agreed to the published version of the manuscript.

Funding: N. Rompotis' research was funded by the UKRI STFC Research Grant ST/S000879/1.

Institutional Review Board Statement: Not applicable.

Informed Consent Statement: Not applicable.

Data Availability Statement: No new data were created or analysed in this study. Data sharing is not applicable to this article.

Conflicts of Interest: The authors declare no conflict of interest.

\section{References}

1. ATLAS Collaboration. Observation of a new particle in the search for the Standard Model Higgs boson with the ATLAS detector at the LHC. Phys. Lett. B 2012, 716, 1-29. [CrossRef]

2. CMS Collaboration. Observation of a New Boson at a Mass of $125 \mathrm{GeV}$ with the CMS Experiment at the LHC. Phys. Lett. B 2012, 716, 30-61. [CrossRef]

3. ATLAS and CMS Collaborations. Combined Measurement of the Higgs Boson Mass in $p p$ Collisions at $\sqrt{s}=7$ and 8 TeV with the ATLAS and CMS Experiments. Phys. Rev. Lett. 2015, 114, 191803. [CrossRef] [PubMed]

4. Evans, L.; Bryant, P. LHC Machine. JINST 2008, 3, S08001. [CrossRef]

5. ATLAS Collaboration. The ATLAS Experiment at the CERN Large Hadron Collider. JINST 2008, 3, S08003. [CrossRef]

6. CMS Collaboration. The CMS Experiment at the CERN LHC. JINST 2008, 3, S08004. [CrossRef]

7. Englert, F.; Brout, R. Broken Symmetry and the Mass of Gauge Vector Mesons. Phys. Rev. Lett. 1964, 13, 321-323. [CrossRef] 
8. Higgs, P.W. Broken symmetries, massless particles and gauge fields. Phys. Lett. 1964, 12, 132-133. [CrossRef]

9. Higgs, P.W. Broken Symmetries and the Masses of Gauge Bosons. Phys. Rev. Lett. 1964, 13, 508-509. [CrossRef]

10. Guralnik, G.S.; Hagen, C.R.; Kibble, T.W.B. Global Conservation Laws and Massless Particles. Phys. Rev. Lett. 1964, 13, 585-587. [CrossRef]

11. Higgs, P.W. Spontaneous Symmetry Breakdown without Massless Bosons. Phys. Rev. 1966, 145, 1156-1163. [CrossRef]

12. Kibble, T.W.B. Symmetry breaking in nonAbelian gauge theories. Phys. Rev. 1967, 155, 1554-1561. [CrossRef]

13. Langacker, P. The Standard Model and Beyond, 2nd ed.; Series in high energy physics, cosmology and gravitation; CRC Press: Boca Raton, FL, USA, 2017. [CrossRef]

14. Particle Data Group. Review of Particle Physics. PTEP 2020, 2020, 083C01. [CrossRef]

15. Barger, V.; Langacker, P.; McCaskey, M.; Ramsey-Musolf, M.; Shaughnessy, G. Complex Singlet Extension of the Standard Model. Phys. Rev. D 2009, 79, 015018. [CrossRef]

16. Barger, V.; Langacker, P.; McCaskey, M.; Ramsey-Musolf, M.J.; Shaughnessy, G. LHC Phenomenology of an Extended Standard Model with a Real Scalar Singlet. Phys. Rev. D 2008, 77, 035005. [CrossRef]

17. Datta, A.; Raychaudhuri, A. Next-to-minimal Higgs: Mass bounds and search prospects. Phys. Rev. D 1998, 57, 2940-2948. [CrossRef]

18. Lee, T.D. A Theory of Spontaneous T Violation. Phys. Rev. D 1973, 8, 1226-1239. [CrossRef]

19. Branco, G.C.; Ferreira, P.M.; Lavoura, L.; Rebelo, M.N.; Sher, M.; Silva, J.P. Theory and phenomenology of two-Higgs-doublet models. Phys. Rept. 2012, 516, 1-102. [CrossRef]

20. Coimbra, R.; Sampaio, M.O.P.; Santos, R. ScannerS: Constraining the phase diagram of a complex scalar singlet at the LHC. Eur. Phys. J. C 2013, 73, 2428. [CrossRef]

21. ATLAS Collaboration. Combined measurements of Higgs boson production and decay using up to $80 \mathrm{fb}^{-1}$ of proton-proton collision data at $\sqrt{s}=13 \mathrm{TeV}$ collected with the ATLAS experiment. Phys. Rev. D 2020, 101, 012002. [CrossRef]

22. CMS Collaboration. Combined measurements of Higgs boson couplings in proton-proton collisions at $\sqrt{s}=13 \mathrm{TeV}$. Eur. Phys. J. C 2019, 79, 421. [CrossRef]

23. ATLAS Collaboration. Combination of searches for Higgs boson pairs in $p p$ collisions at $\sqrt{s}=13$ TeV with the ATLAS detector. Phys. Lett. B 2020, 800, 135103. [CrossRef]

24. CMS Collaboration. Combination of searches for Higgs boson pair production in proton-proton collisions at $\sqrt{s}=13 \mathrm{TeV}$. Phys. Rev. Lett. 2019, 122, 121803. [CrossRef]

25. Silveira, V.; Zee, A. Scalar Phantoms. Phys. Lett. B 1985, 161, 136-140. [CrossRef]

26. Costa, R.; Mühlleitner, M.; Sampaio, M.O.P.; Santos, R. Singlet Extensions of the Standard Model at LHC Run 2: Benchmarks and Comparison with the NMSSM. JHEP 2016, 06, 034. [CrossRef]

27. Profumo, S.; Ramsey-Musolf, M.J.; Shaughnessy, G. Singlet Higgs phenomenology and the electroweak phase transition. JHEP 2007, 8, 010. [CrossRef]

28. Kajantie, K.; Laine, M.; Rummukainen, K.; Shaposhnikov, M.E. Is there a hot electroweak phase transition at $m_{H} \gtrsim m_{W}$ ? Phys. Rev. Lett. 1996, 77, 2887-2890. [CrossRef]

29. Damgaard, P.H.; O'Connell, D.; Petersen, T.C.; Tranberg, A. Constraints on New Physics from Baryogenesis and Large Hadron Collider Data. Phys. Rev. Lett. 2013, 111, 221804. [CrossRef]

30. Djouadi, A. The Anatomy of electro-weak symmetry breaking. II. The Higgs bosons in the minimal supersymmetric model. Phys. Rept. 2008, 459, 1-241. [CrossRef]

31. Kim, J.E. Light Pseudoscalars, Particle Physics and Cosmology. Phys. Rept. 1987, 150, 1-177. [CrossRef]

32. Glashow, S.L.; Weinberg, S. Natural Conservation Laws for Neutral Currents. Phys. Rev. D 1977, 15, 1958. [CrossRef]

33. Paschos, E.A. Diagonal Neutral Currents. Phys. Rev. D 1977, 15, 1966. [CrossRef]

34. Gunion, J.F.; Haber, H.E. The CP conserving two Higgs doublet model: the approach to the decoupling limit. Phys. Rev. D 2003, 67, 075019. [CrossRef]

35. LHC Higgs Cross Section Working Group. Handbook of LHC Higgs Cross Sections: 4. Deciphering the Nature of the Higgs Sector. CERN Yellow Rep. Monogr. 2017, 2. [CrossRef]

36. Basler, P.; Krause, M.; Mühlleitner, M.; Wittbrodt, J.; Wlotzka, A. Strong First Order Electroweak Phase Transition in the CP-Conserving 2HDM Revisited. JHEP 2017, 2, 121. [CrossRef]

37. Fontes, D.; Romão, J.C.; Santos, R.; Silva, J.A.P. Undoubtable signs of CP-violation in Higgs boson decays at the LHC run 2. Phys. Rev. D 2015, 92, 055014. [CrossRef]

38. Carena, M.; Heinemeyer, S.; Wagner, C.E.M.; Weiglein, G. Suggestions for Benchmark Scenarios for MSSM Higgs Boson Searches at Hadron Colliders. Eur. Phys. J. C 2003, 26, 601-607. [CrossRef]

39. Carena, M.; Heinemeyer, S.; Stål, O.; Wagner, C.E.M.; Weiglein, G. MSSM Higgs Boson Searches at the LHC: Benchmark Scenarios after the Discovery of a Higgs-like Particle. Eur. Phys. J. C 2013, 73, 2552. [CrossRef]

40. Bagnaschi, E.; others. MSSM Higgs Boson Searches at the LHC: Benchmark Scenarios for Run 2 and Beyond. Eur. Phys. J. C 2019, 79, 617. [CrossRef]

41. Maiani, L.; Polosa, A.D.; Riquer, V. Bounds to the Higgs Sector Masses in Minimal Supersymmetry from LHC Data. Phys. Lett. B 2013, 724, 274-277. [CrossRef] 
42. Djouadi, A.; Quevillon, J. The MSSM Higgs sector at a high $M_{S U S Y}$ : reopening the low $\tan \beta$ regime and heavy Higgs searches. JHEP 2013, 10, 028. [CrossRef]

43. Djouadi, A.; Maiani, L.; Moreau, G.; Polosa, A.; Quevillon, J.; Riquer, V. The post-Higgs MSSM scenario: Habemus MSSM? Eur. Phys. J. C 2013, 73, 2650. [CrossRef]

44. Djouadi, A.; Maiani, L.; Polosa, A.; Quevillon, J.; Riquer, V. Fully covering the MSSM Higgs sector at the LHC. JHEP 2015, 6, 168, [CrossRef]

45. Mühlleitner, M.; Sampaio, M.O.P.; Santos, R.; Wittbrodt, J. ScannerS: Parameter Scans in Extended Scalar Sectors. 2020. Available online: https:/ / gitlab.com/jonaswittbrodt/ScannerS (accessed on 25 October 2021).

46. Mühlleitner, M.; Sampaio, M.O.P.; Santos, R.; Wittbrodt, J. The N2HDM under Theoretical and Experimental Scrutiny. JHEP 2017, 3, 94. [CrossRef]

47. Maniatis, M. The Next-to-Minimal Supersymmetric extension of the Standard Model reviewed. Int. J. Mod. Phys. A 2010, 25, 3505-3602. [CrossRef]

48. Arhrib, A.; Benbrik, R.; Moretti, S. Bosonic Decays of Charged Higgs Bosons in a 2HDM Type-I. Eur. Phys. J. C 2017, 77, 621, [CrossRef]

49. Kling, F.; Pyarelal, A.; Su, S. Light Charged Higgs Bosons to AW/HW via Top Decay. JHEP 2015, 11, 051. [CrossRef]

50. Bahl, H.; Stefaniak, T.; Wittbrodt, J. The forgotten channels: charged Higgs boson decays to a $\mathrm{W}^{ \pm}$and a non-SM-like Higgs boson. JHEP 2021, 6, 183. [CrossRef]

51. Rathsman, J.; Rossler, T. Closing the Window on Light Charged Higgs Bosons in the NMSSM. Adv. High Energy Phys. 2012, 2012, 853706. [CrossRef]

52. Georgi, H.; Machacek, M. Doubly Charged Higgs Bosons. Nucl. Phys. B 1985, 262, 463-477. [CrossRef]

53. Hartling, K.; Kumar, K.; Logan, H.E. The decoupling limit in the Georgi-Machacek model. Phys. Rev. D 2014, 90, 015007, [CrossRef]

54. LHC Higgs Cross Section Working Group. Handbook of LHC Higgs Cross Sections: 1. Inclusive Observables. CERN Yellow Rep. Monogr. 2011, 2. [CrossRef]

55. LHC Higgs Cross Section Working Group. Handbook of LHC Higgs Cross Sections: 2. Differential Distributions. CERN Yellow Rep. Monogr. 2012, 2. [CrossRef]

56. LHC Higgs Cross Section Working Group. Handbook of LHC Higgs Cross Sections: 3. Higgs Properties. CERN Yellow Rep. Monogr. 2013, 4, [CrossRef]

57. Djouadi, A.; Kalinowski, J.; Spira, M. HDECAY: A Program for Higgs boson decays in the standard model and its supersymmetric extension. Comput. Phys. Commun. 1998, 108, 56-74. [CrossRef]

58. Eriksson, D.; Rathsman, J.; Stål, O. 2HDMC: Two-Higgs-Doublet Model Calculator Physics and Manual. Comput. Phys. Commun. 2010, 181, 189-205. [CrossRef]

59. Hartling, K.; Kumar, K.; Logan, H.E. GMCALC: A Calculator for the Georgi-Machacek Model 2014. Available online: https: //people.physics.carleton.ca/ logan/gmcalc/ (accessed on 25 October 2021).

60. Harlander, R.V.; Liebler, S.; Mantler, H. SusHi: A program for the calculation of Higgs production in gluon fusion and bottom-quark annihilation in the Standard Model and the MSSM. Comput. Phys. Commun. 2013, 184, 1605-1617. [CrossRef]

61. Harlander, R.V.; Liebler, S.; Mantler, H. SusHi Bento: Beyond NNLO and the heavy-top limit. Comput. Phys. Commun. 2017, 212, 239-257. [CrossRef]

62. Dawson, S.; Jackson, C.B.; Reina, L.; Wackeroth, D. Exclusive Higgs boson production with bottom quarks at hadron colliders. Phys. Rev. D 2004, 69, 074027, [CrossRef]

63. Dittmaier, S.; Krämer, M.; Spira, M. Higgs radiation off bottom quarks at the Tevatron and the CERN LHC. Phys. Rev. D 2004, 70, 074010. [CrossRef]

64. Harlander, R.V.; Krämer, M.; Schumacher, M. Bottom-Quark Associated Higgs-Boson Production: Reconciling the Four- and Five-Flavour Scheme Approach. CERN-PH-TH-2011-134. 2011. Available online: https://cds.cern.ch/record/1407669 (accessed on 25 October 2021).

65. Bonvini, M.; Papanastasiou, A.S.; Tackmann, F.J. Resummation and matching of b-quark mass effects in $b \bar{b} H$ production. JHEP 2015, 11, 196. [CrossRef]

66. Bonvini, M.; Papanastasiou, A.S.; Tackmann, F.J. Matched predictions for the $b \bar{b} H$ cross section at the 13 TeV LHC. JHEP 2016, 10, 053. [CrossRef]

67. Forte, S.; Napoletano, D.; Ubiali, M. Higgs production in bottom-quark fusion in a matched scheme. Phys. Lett. B 2015, 751, 331-337. [CrossRef]

68. Forte, S.; Napoletano, D.; Ubiali, M. Higgs production in bottom-quark fusion: matching beyond leading order. Phys. Lett. B 2016, 763, 190-196. [CrossRef]

69. Belyaev, A.; Garcia, D.; Guasch, J.; Sola, J. Prospects for supersymmetric charged Higgs boson discovery at the Tevatron and the CERN LHC. Phys. Rev. D 2002, 65, 031701. [CrossRef]

70. Belyaev, A.; Garcia, D.; Guasch, J.; Sola, J. Prospects for heavy supersymmetric charged Higgs boson searches at hadron colliders. JHEP 2002, 6, 059. [CrossRef]

71. Zhu, S.h. Complete next-to-leading order QCD corrections to charged Higgs boson associated production with top quark at the CERN large hadron collider. Phys. Rev. D 2003, 67, 075006. [CrossRef] 
72. Gao, G.p.; Lu, G.r.; Xiong, Z.h.; Yang, J.M. Loop effects and non-decoupling property of SUSY QCD in $g b \rightarrow t H^{-}$. Phys. Rev. D 2002, 66, 015007. [CrossRef]

73. Plehn, T. Charged Higgs boson production in bottom gluon fusion. Phys. Rev. D 2003, 67, 014018. [CrossRef]

74. Berger, E.L.; Han, T.; Jiang, J.; Plehn, T. Associated production of a top quark and a charged Higgs boson. Phys. Rev. D 2005, 71, 115012. [CrossRef]

75. Wu, P.; Ma, W.G.; Zhang, R.Y.; Jiang, Y.; Han, L.; Guo, L. NLO supersymmetric QCD corrections to the $t \bar{b} H^{-}$associated production at hadron colliders. Phys. Rev. D 2006, 73, 015012. [CrossRef]

76. Dittmaier, S.; Krämer, M.; Spira, M.; Walser, M. Charged-Higgs-boson production at the LHC: NLO supersymmetric QCD corrections. Phys. Rev. D 2011, 83, 055005. [CrossRef]

77. Flechl, M.; Klees, R.; Krämer, M.; Spira, M.; Ubiali, M. Improved cross-section predictions for heavy charged Higgs boson production at the LHC. Phys. Rev. D 2015, 91, 075015. [CrossRef]

78. Degrande, C.; Ubiali, M.; Wiesemann, M.; Zaro, M. Heavy charged Higgs boson production at the LHC. JHEP 2015, 10, 145. [CrossRef]

79. Degrande, C.; Frederix, R.; Hirschi, V.; Ubiali, M.; Wiesemann, M.; Zaro, M. Accurate predictions for charged Higgs production: Closing the $m_{H^{ \pm}} \sim m_{t}$ window. Phys. Lett. B 2017, 772, 87-92. [CrossRef]

80. Kidonakis, N. Charged Higgs production in association with a top quark at approximate NNLO. Phys. Rev. D 2016, 94, 014010. [CrossRef]

81. Veatch, J. Searches for resonant scalar boson pair production using Run 2 LHC proton-proton collision data. Symmetry 2021, in press.

82. ATLAS Collaboration. Search for heavy Higgs bosons decaying into two tau leptons with the ATLAS detector using $p p$ collisions at $\sqrt{s}=13$ TeV. Phys. Rev. Lett. 2020, 125, 051801. [CrossRef]

83. CMS Collaboration. Search for additional neutral MSSM Higgs bosons in the $\tau \tau$ final state in proton-proton collisions at $\sqrt{s}=$ $13 \mathrm{TeV}$. JHEP 2018, 9, 007. [CrossRef]

84. CMS Collaboration. Evidence for the $125 \mathrm{GeV}$ Higgs boson decaying to a pair of $\tau$ leptons. JHEP 2014, 5, 104. [CrossRef]

85. ATLAS Collaboration. Modelling $Z \rightarrow \tau \tau$ processes in ATLAS with $\tau$-embedded $Z \rightarrow \mu \mu$ data. JINST 2015, 10, P09018. [CrossRef]

86. ATLAS Collaboration. Search for the neutral Higgs bosons of the Minimal Supersymmetric Standard Model in $p p$ collisions at $\sqrt{s}=7 \mathrm{TeV}$ with the ATLAS detector. JHEP 2013, 2, 095. [CrossRef]

87. Bechtle, P.; Brein, O.; Heinemeyer, S.; Weiglein, G.; Williams, K.E. HiggsBounds: Confronting Arbitrary Higgs Sectors with Exclusion Bounds from LEP and the Tevatron. Comput. Phys. Commun. 2010, 181, 138-167. [CrossRef]

88. ATLAS Collaboration. Search for a heavy Higgs boson decaying into a Z boson and another heavy Higgs boson in the $\ell \ell b b$ and $\ell \ell W W$ final states in $p p$ collisions at $\sqrt{s}=13 \mathrm{TeV}$ with the ATLAS detector. Eur. Phys. J. C 2021, 81, 396. [CrossRef]

89. ATLAS Collaboration. Search for heavy neutral Higgs bosons produced in association with $b$-quarks and decaying into $b$-quarks at $\sqrt{s}=13 \mathrm{TeV}$ with the ATLAS detector. Phys. Rev. D 2020, 102, 032004. [CrossRef]

90. CMS Collaboration. Search for neutral MSSM Higgs bosons decaying into a pair of bottom quarks. JHEP 2015, 11, 071. [CrossRef]

91. ATLAS Collaboration. Search for scalar resonances decaying into $\mu^{+} \mu^{-}$in events with and without $b$-tagged jets produced in proton-proton collisions at $\sqrt{s}=13 \mathrm{TeV}$ with the ATLAS detector. JHEP 2019, 7, 117. [CrossRef]

92. CMS Collaboration. Search for MSSM Higgs bosons decaying to $\mu^{+} \mu^{-}$in proton-proton collisions at $\sqrt{s}=13 \mathrm{TeV}$. Phys. Lett. B 2019, 798, 134992. [CrossRef]

93. ATLAS Collaboration. Search for Heavy Higgs Bosons $A / H$ Decaying to a Top Quark Pair in $p p$ Collisions at $\sqrt{s}=8$ TeV with the ATLAS Detector. Phys. Rev. Lett. 2017, 119, 191803. [CrossRef]

94. CMS Collaboration. Search for heavy Higgs bosons decaying to a top quark pair in proton-proton collisions at $\sqrt{s}=13 \mathrm{TeV}$. JHEP 2020, 4, 171. [CrossRef]

95. Randall, L.; Sundrum, R. A Large mass hierarchy from a small extra dimension. Phys. Rev. Lett. 1999, 83, 3370-3373. [CrossRef]

96. Randall, L.; Sundrum, R. An Alternative to compactification. Phys. Rev. Lett. 1999, 83, 4690-4693. [CrossRef]

97. ATLAS Collaboration. Search for resonances decaying into photon pairs in $139 \mathrm{fb}^{-1}$ of pp collisions at $\sqrt{s}=13 \mathrm{TeV}$ with the ATLAS detector. Phys. Lett. B 2021, 822, 136651. [CrossRef]

98. CMS Collaboration. Search for physics beyond the standard model in high-mass diphoton events from proton-proton collisions at $\sqrt{s}=13 \mathrm{TeV}$. Phys. Rev. D 2018, 98, 092001. [CrossRef]

99. CMS Collaboration. Search for a standard model-like Higgs boson in the mass range between 70 and $110 \mathrm{GeV}$ in the diphoton final state in proton-proton collisions at $\sqrt{s}=8$ and 13 TeV. Phys. Lett. B 2019, 793, 320-347. [CrossRef]

100. ATLAS Collaboration. Search for heavy ZZ resonances in the $\ell^{+} \ell^{-} \ell^{+} \ell^{-}$and $\ell^{+} \ell^{-} v \bar{v}$ final states using proton-proton collisions at $\sqrt{s}=13 \mathrm{TeV}$ with the ATLAS detector. Eur. Phys. J. C 2018, 78, 293. [CrossRef]

101. ATLAS Collaboration. Searches for heavy ZZ and ZW resonances in the $\ell \ell q q$ and $v v q q$ final states in $p p$ collisions at $\sqrt{s}=13$ TeV with the ATLAS detector. JHEP 2018, 3, 009. [CrossRef]

102. ATLAS Collaboration. Search for heavy resonances decaying into $W W$ in the $e v \mu v$ final state in $p p$ collisions at $\sqrt{s}=13 \mathrm{TeV}$ with the ATLAS detector. Eur. Phys. J. C 2018, 78, 24. [CrossRef]

103. ATLAS Collaboration. Search for $W W / W Z$ resonance production in $\ell v q q$ final states in $p p$ collisions at $\sqrt{s}=13$ TeV with the ATLAS detector. JHEP 2018, 3, 042. [CrossRef] 
104. ATLAS Collaboration. Combination of searches for heavy resonances decaying into bosonic and leptonic final states using $36 \mathrm{fb}^{-1}$ of proton-proton collision data at $\sqrt{s}=13 \mathrm{TeV}$ with the ATLAS detector. Phys. Rev. D 2018, 98, 052008. [CrossRef]

105. CMS Collaboration. Search for a new scalar resonance decaying to a pair of $Z$ bosons in proton-proton collisions at $\sqrt{s}=13 \mathrm{TeV}$. JHEP 2018, 6, 127. [CrossRef]

106. CMS Collaboration. Search for a heavy Higgs boson decaying to a pair of $\mathrm{W}$ bosons in proton-proton collisions at $\sqrt{\mathrm{s}}=13 \mathrm{TeV}$. JHEP 2020, 3, 34. [CrossRef]

107. ATLAS Collaboration. Search for heavy resonances decaying into a pair of $Z$ bosons in the $\ell^{+} \ell^{-} \ell^{\prime+} \ell^{\prime-}$ and $\ell^{+} \ell^{-} \nu \bar{v}$ final states using $139 \mathrm{fb}^{-1}$ of proton-proton collisions at $\sqrt{s}=13 \mathrm{TeV}$ with the ATLAS detector. Eur. Phys. J. C 2021, 81, 332. [CrossRef]

108. CMS Collaboration. Search for new neutral Higgs bosons through the $\mathrm{H} \rightarrow \mathrm{ZA} \rightarrow \ell^{+} \ell^{-} \mathrm{b} \overline{\mathrm{b}}$ process in pp collisions at $\sqrt{s}=13$ TeV. JHEP 2020, 3, 55. [CrossRef]

109. CMS Collaboration. Search for neutral resonances decaying into a $Z$ boson and a pair of $\mathrm{b}$ jets or $\tau$ leptons. Phys. Lett. $B$ 2016, 759, 369-394. [CrossRef]

110. ATLAS Collaboration. Search for Heavy Resonances Decaying into a Z Boson and a Higgs Boson in Final States with Leptons and $b$-jets in $139 \mathrm{fb}^{-1}$ of $p p$ Collisions at $\sqrt{s}=13 \mathrm{TeV}$ with the ATLAS Detector. ATLAS-CONF-2020-043. 2020. Available online: http://cdsweb.cern.ch/record/2728053 (accessed on 25 October 2021).

111. CMS Collaboration. Search for a heavy pseudoscalar boson decaying to a Z and a Higgs boson at $\sqrt{s}=13 \mathrm{TeV}$. Eur. Phys. J. C 2019, 79, 564. [CrossRef] [PubMed]

112. CMS Collaboration. Search for a heavy pseudoscalar Higgs boson decaying into a $125 \mathrm{GeV}$ Higgs boson and a Z boson in final states with two tau and two light leptons at $\sqrt{s}=13 \mathrm{TeV}$. JHEP 2020, 3, 065. [CrossRef]

113. Pappadopulo, D.; Thamm, A.; Torre, R.; Wulzer, A. Heavy Vector Triplets: Bridging Theory and Data. JHEP 2014, 9, 060. [CrossRef]

114. ATLAS Collaboration. hMSSM Summary Plots from Direct and Indirect Searches. ATL-PHYS-PUB-2021-030. 2021. Available online: http:/ / cdsweb.cern.ch/record/2777012 (accessed on 25 October 2021).

115. ATLAS Collaboration. A Search for Light CP-Odd Higgs Bosons Decaying to mu+ mu- in ATLAS. ATLAS-CONF-2011-020. 2021. Available online: https:/ / cds.cern.ch/record/1336749 (accessed on 25 October 2021).

116. CMS Collaboration. Search for a Light Pseudoscalar Higgs Boson in the Dimuon Decay Channel in $p p$ Collisions at $\sqrt{s}=7$ TeV. Phys. Rev. Lett. 2012, 109, 121801. [CrossRef]

117. ATLAS Collaboration. Search for Higgs Boson Decays into a Pair of Pseudoscalar Particles in the $b b \mu \mu$ Final State with the ATLAS Detector in $p p$ Collisions at $\sqrt{s}=13 \mathrm{TeV}$. 2021. Available online: http:/ /xxx.lanl.gov/abs/2110.00313 (accessed on 25 October 2021).

118. CMS Collaboration. Search for an exotic decay of the Higgs boson to a pair of light pseudoscalars in the final state with two muons and two b quarks in pp collisions at 13 TeV. Phys. Lett. B 2019, 795, 398-423. [CrossRef]

119. Curtin, D.; others. Exotic decays of the $125 \mathrm{GeV}$ Higgs boson. Phys. Rev. D 2014, 90, 075004. [CrossRef]

120. Haisch, U.; Kamenik, J.F.; Malinauskas, A.; Spira, M. Collider constraints on light pseudoscalars. JHEP 2018, 3, 178. [CrossRef]

121. ATLAS Collaboration. Search for Higgs boson decays to beyond-the-Standard-Model light bosons in four-lepton events with the ATLAS detector at $\sqrt{s}=13 \mathrm{TeV}$. JHEP 2018, 6, 166. [CrossRef]

122. CMS Collaboration. A search for pair production of new light bosons decaying into muons in proton-proton collisions at 13 TeV. Phys. Lett. B 2019, 796, 131-154. [CrossRef]

123. CMS Collaboration. Search for light pseudoscalar boson pairs produced from decays of the $125 \mathrm{GeV}$ Higgs boson in final states with two muons and two nearby tracks in pp collisions at $\sqrt{s}=13 \mathrm{TeV}$. Phys. Lett. B 2020, 800, 135087. [CrossRef]

124. ATLAS Collaboration. Search for Higgs bosons decaying to $a a$ in the $\mu \mu \tau \tau$ final state in $p p$ collisions at $\sqrt{s}=8 \mathrm{TeV}$ with the ATLAS experiment. Phys. Rev. D 2015, 92, 052002. [CrossRef]

125. CMS Collaboration. Search for an exotic decay of the Higgs boson to a pair of light pseudoscalars in the final state of two muons and two $\tau$ leptons in proton-proton collisions at $\sqrt{s}=13 \mathrm{TeV}$. JHEP 2018, 11, 018. [CrossRef]

126. CMS Collaboration. Search for a light pseudoscalar Higgs boson in the boosted $\mu \mu \tau \tau$ final state in proton-proton collisions at $\sqrt{s}=13 \mathrm{TeV}$. JHEP 2020, 8, 139. [CrossRef]

127. CMS Collaboration. Search for an exotic decay of the Higgs boson to a pair of light pseudoscalars in the final state with two $b$ quarks and two $\tau$ leptons in proton-proton collisions at $\sqrt{s}=13 \mathrm{TeV}$. Phys. Lett. B 2018, 785, 462. [CrossRef]

128. ATLAS Collaboration. Search for Higgs boson decays into two new low-mass spin-zero particles in the $4 b$ channel with the ATLAS detector using $p p$ collisions at $\sqrt{s}=13 \mathrm{TeV}$. Phys. Rev. D 2020, 102, 112006. [CrossRef]

129. ATLAS Collaboration. Search for new phenomena in events with at least three photons collected in $p p$ collisions at $\sqrt{s}=8 \mathrm{TeV}$ with the ATLAS detector. Eur. Phys. J. C 2016, 76, 210. [CrossRef] [PubMed]

130. CMS Collaboration. Search for Exotic Decay of the Higgs Boson into Two Light Pseudoscalars with Four Photons in the Final State at $\sqrt{s}=13$ TeV. CMS-PAS-HIG-21-003. 2021. Available online: https://cds.cern.ch/record/2776775 (accessed on 25 October 2021).

131. ATLAS Collaboration. Search for Higgs boson decays into pairs of light (pseudo)scalar particles in the $\gamma \gamma j j$ final state in $p p$ collisions at $\sqrt{s}=13 \mathrm{TeV}$ with the ATLAS detector. Phys. Lett. B 2018, 782, 750-767. [CrossRef]

132. CMS Collaboration. Available online: https://twiki.cern.ch/twiki/bin/view/CMSPublic/Summary2HDMSRun2 (accessed on 6 November 2021). 
133. ATLAS Collaboration. Summary of Exotic Higgs Boson Decays from the ATLAS Experiment. ATL-PHYS-PUB-2021-008. 2021. Available online: https://cds.cern.ch/record/2758783 (accessed on 25 October 2021).

134. ATLAS Collaboration. Search for charged Higgs bosons decaying via $H^{ \pm} \rightarrow \tau^{ \pm} v_{\tau}$ in the $\tau+$ jets and $\tau+$ lepton final states with 36 $\mathrm{fb}^{-1}$ of $p p$ collision data recorded at $\sqrt{s}=13 \mathrm{TeV}$ with the ATLAS experiment. JHEP 2018, 9, 139. [CrossRef]

135. CMS Collaboration. Search for charged Higgs bosons in the $\mathrm{H}^{ \pm} \rightarrow \tau^{ \pm} v_{\tau}$ decay channel in proton-proton collisions at $\sqrt{s}=13$ TeV. JHEP 2019, 7, 142. [CrossRef]

136. ATLAS Collaboration. Search for charged Higgs bosons decaying into top and bottom quarks at $\sqrt{s}=13$ TeV with the ATLAS detector. JHEP 2018, 11, 085. [CrossRef]

137. ATLAS Collaboration. Search for charged Higgs bosons decaying into a top quark and a bottom quark at $\sqrt{\mathrm{s}}=13$ TeV with the ATLAS detector. JHEP 2021, 6, 145. [CrossRef]

138. CMS Collaboration. Search for a charged Higgs boson decaying into top and bottom quarks in events with electrons or muons in proton-proton collisions at $\sqrt{\mathrm{s}}=13 \mathrm{TeV}$. JHEP 2020, 1, 096. [CrossRef]

139. CMS Collaboration. Search for charged Higgs bosons decaying into a top and a bottom quark in the all-jet final state of pp collisions at $\sqrt{s}=13 \mathrm{TeV}$. JHEP 2020, 7, 126. [CrossRef]

140. ATLAS Collaboration. Search for a Light Charged Higgs Boson in $t \rightarrow H^{+} b$ Decays, with $H^{+} \rightarrow c b$, in the Lepton+Jets Final State in Proton-Proton Collisions at $\sqrt{s}=13 \mathrm{TeV}$ with the ATLAS Detector. ATLAS-CONF-2021-037. 2021. Available online: https: / /cds.cern.ch/record/2779169 (accessed on 25 October 2021).

141. CMS Collaboration. Search for a light charged Higgs boson in the $H^{ \pm} \rightarrow$ cs channel in proton-proton collisions at $\sqrt{s}=13 \mathrm{TeV}$. Phys. Rev. D 2020, 102, 072001. [CrossRef]

142. Akeroyd, A.G.; Moretti, S.; Yagyu, K.; Yildirim, E. Light charged Higgs boson scenario in 3-Higgs doublet models. Int. J. Mod. Phys. A 2017, 32, 1750145. [CrossRef]

143. Akeroyd, A.G.; Moretti, S.; Song, M. Light charged Higgs boson with dominant decay to quarks and its search at the LHC and future colliders. Phys. Rev. D 2018, 98, 115024. [CrossRef]

144. CMS Collaboration. Search for a light charged Higgs boson decaying to a W boson and a CP-odd Higgs boson in final states with e $\mu \mu$ or $\mu \mu \mu$ in proton-proton collisions at $\sqrt{s}=13 \mathrm{TeV}$. Phys. Rev. Lett. 2019, 123, 131802. [CrossRef]

145. ATLAS Collaboration. Search for $H^{ \pm} \rightarrow W^{ \pm} A \rightarrow W^{ \pm} \mu \mu$ in $p p \rightarrow t \bar{t}$ Events Using an $e \mu \mu$ Signature with the ATLAS Detector at $\sqrt{s}=13$ TeV. ATLAS-CONF-2021-047. 2021. Available online: https://cds.cern.ch/record/2780092 (accessed on 25 October 2021).

146. ATLAS Collaboration. Search for resonant $W Z$ production in the fully leptonic final state in proton-proton collisions at $\sqrt{s}=13$ TeV with the ATLAS detector. Phys. Lett. B 2018, 787, 68-88. [CrossRef]

147. CMS Collaboration. Search for charged Higgs bosons produced in vector boson fusion processes and decaying into vector boson pairs in proton-proton collisions at $\sqrt{s}=13 \mathrm{TeV}$. Eur. Phys. J. C 2021, 81, 723. [CrossRef]

148. ATLAS Collaboration. Search for doubly and singly charged Higgs bosons decaying into vector bosons in multi-lepton final states with the ATLAS detector using proton-proton collisions at $\sqrt{s}=13 \mathrm{TeV}$. JHEP 2021, 6, 146. [CrossRef]

149. Arhrib, A.; Benbrik, R.; Chabab, M.; Moultaka, G.; Peyranere, M.C.; Rahili, L.; Ramadan, J. The Higgs Potential in the Type II Seesaw Model. Phys. Rev. D 2011, 84, 095005. [CrossRef]

150. Schechter, J.; Valle, J.W.F. Neutrino Masses in SU(2) x U(1) Theories. Phys. Rev. D 1980, 22, 2227. [CrossRef]

151. Kanemura, S.; Kikuchi, M.; Yagyu, K.; Yokoya, H. Bounds on the mass of doubly-charged Higgs bosons in the same-sign diboson decay scenario. Phys. Rev. D 2014, 90, 115018. [CrossRef]

152. CMS Collaboration. Available online: https://twiki.cern.ch/twiki/bin/view/CMSPublic/SummaryResultsHIG (accessed on 6 November 2021).

153. Cepeda, M.; others. Report from Working Group 2: Higgs Physics at the HL-LHC and HE-LHC. CERN Yellow Rep. Monogr. 2019, 7, 221-584. [CrossRef]

154. Muon g-2 Collaboration. Measurement of the Positive Muon Anomalous Magnetic Moment to 0.46 ppm. Phys. Rev. Lett. 2021, 126, 141801. [CrossRef]

155. Bernlochner, F.U.; Ligeti, Z.; Papucci, M.; Robinson, D.J. Combined analysis of semileptonic $B$ decays to $D$ and $D^{*}: R\left(D^{(*)}\right),\left|V_{c b}\right|$, and new physics. Phys. Rev. D 2017, 95, 115008. [CrossRef]

156. LHCb Collaboration. Test of lepton universality with $B^{0} \rightarrow K^{* 0} \ell^{+} \ell^{-}$decays. JHEP 2017, 8, 055. [CrossRef] 